\title{
Theory of elastic neutrino-electron scattering
}

\author{
Oleksandr Tomalak $\oplus^{1,2,3, *}$ and Richard J. Hill $\oplus^{1,2, \dagger}$ \\ ${ }^{1}$ Department of Physics and Astronomy, University of Kentucky, Lexington, Kentucky 40506, USA \\ ${ }^{2}$ Theoretical Physics Department, Fermi National Accelerator Laboratory, Batavia, Illinois 60510, USA \\ ${ }^{3}$ Institut für Kernphysik and PRISMA Cluster of Excellence, Johannes Gutenberg Universität, \\ D-55099 Mainz, Germany
}

(Received 19 July 2019; accepted 15 January 2020; published 21 February 2020)

\begin{abstract}
Theoretical predictions for elastic neutrino-electron scattering have no hadronic or nuclear uncertainties at leading order making this process an important tool for normalizing neutrino flux. However, the process is subject to large radiative corrections that differ according to experimental conditions. In this paper, we collect new and existing results for total and differential cross sections accompanied by radiation of one photon, $\nu e \rightarrow \nu e(\gamma)$. We perform calculations within the Fermi effective theory and provide analytic expressions for the electron energy spectrum and for the total electromagnetic energy spectrum as well as for double- and triple-differential cross sections with respect to electron energy, electron angle, photon energy, and photon angle. We discuss illustrative applications to accelerator-based neutrino experiments and provide the most precise up-to-date values of neutrino-electron scattering cross sections. We present an analysis of theoretical error, which is dominated by the $\sim 0.2 \%-0.4 \%$ uncertainty of the hadronic correction. We also discuss how searches for new physics can be affected by radiative corrections.
\end{abstract}

DOI: 10.1103/PhysRevD.101.033006

\section{INTRODUCTION}

In the Standard Model of particle physics, neutrinos are massless particles. However, experiments with solar [1-6], atmospheric [7,8], reactor [9-13], and accelerator [14-16] neutrinos ${ }^{1}$ establish that neutrinos oscillate and have nonzero mass $[17,18]$, thus providing a convincing example of physics beyond the Standard Model. Fundamental questions about this definitive portal to new physics remain unanswered: What is the origin of neutrino mass? Are lepton number and $C P$ symmetries violated? Do sterile neutrinos exist? What is the absolute scale and ordering of neutrino masses? New experiments aim to address these questions but rely on a precise description of neutrino interactions with the ordinary matter (electrons and nuclei) used to detect them.

Interactions with atomic nuclei compose the bulk of neutrino scattering events at accelerator neutrino experiments. Although interactions with atomic electrons are rarer, they are nonetheless valuable. The neutrino-electron

\footnotetext{
*oleksandr.tomalak@uky.edu

†Richard.Hill@uky.edu

${ }^{1}$ For the purposes of this paper, "accelerator" neutrinos have energy large compared to the electron mass.

Published by the American Physical Society under the terms of the Creative Commons Attribution 4.0 International license. Further distribution of this work must maintain attribution to the author(s) and the published article's title, journal citation, and DOI. Funded by SCOAP ${ }^{3}$.
}

scattering process plays an important dual role: first, owing to a clean experimental signature and a small cross-section uncertainty, the process provides an incisive constraint on neutrino flux $[19,20]$; second, the bulk of next-toleading order (NLO) radiative corrections can be evaluated analytically and thus serve as a prototype for the more complicated cases of neutrino-nucleon and neutrinonucleus scattering.

Radiative corrections to elastic neutrino-electron scattering of order $\alpha$ were calculated first in Ref. [21], where only soft-photon bremsstrahlung was considered. In Ref. [22], an analytical phase-space integration technique was developed to include hard-photon bremsstrahlung, and the electron energy spectrum for neutrinoelectron scattering accompanied by one radiated photon was obtained. The leading-order (LO) cross section in the low-energy limit of the Weinberg theory [23] was evaluated in Ref. [24]. References [25,26] presented the electron energy spectrum in the limit of small electron mass accounting for corrections of order $\alpha$ and including other electroweak NLO radiative corrections. The electromagnetic energy spectrum was considered in Refs. [27,28]. Reference [29] reproduced results of Refs. $[22,25]$ by numerically performing the phase-space integration, and accounted for the electron mass suppressed interference term; Ref. [29] also presented a numerical evaluation of the electromagnetic energy spectrum. The hard-photon correction to the total elastic cross section was studied in Refs. [30,31]. Different aspects of 
radiative corrections in elastic neutrino-electron scattering were also discussed in Refs. [27-44]. See Refs. [45,46] for recent reviews.

In this work, we analytically evaluate relevant distributions and spectra in elastic (anti)neutrino-electron scattering starting from four-fermion effective field theory (EFT). We take neutrino-lepton and neutrino-quark EFT coefficients from Ref. [47] (with $n_{f}=4$ active quarks at renormalization scale $\mu=2 \mathrm{GeV}$ ) and calculate real and virtual corrections in the $\overline{\mathrm{MS}}$ renormalization scheme within this theory. Exploiting the technique of Ref. [22], we evaluate the electron energy spectrum and present this calculation in a relatively compact form. We generalize this technique for the evaluation of the electromagnetic energy spectrum as well as triple- and double-differential cross sections. We discuss a new treatment of hadronic loop diagrams; this contribution dominates the error budget for neutrino-electron scattering and impacts other neutral current neutrino processes, such as coherent neutrinonucleus scattering [48]. As illustrative applications using accelerator neutrino beams [16,49-51], we consider the impact of radiative corrections on energy spectra and compare observables employing electron energy vs total electromagnetic energy. For possible low-energy applications, we provide results in analytic form keeping all charged lepton mass terms. The complete mass dependence could be useful in the analysis of future reactor and solar neutrino experiments [52-56]. We also discuss examples where radiative corrections can impact searches for new physics, including neutrino charge radius effects.

The paper is organized as follows. Section II considers the kinematics of neutrino-electron scattering and computes the tree-level scattering process including electroweak corrections to the low-energy four-fermion interaction. Section III computes virtual corrections to elastic scattering. Section IV represents the bulk of the paper and computes QED corrections involving real radiation. Section V presents illustrative results for total cross sections and electron energy vs total electromagnetic energy spectra. Section VI presents our conclusions and outlook. In the main text of the paper, we describe the general strategy of the computations and focus on results in the limit of small electron mass (i.e., neutrino beam energy much larger than electron mass). Appendixes provide general expressions retaining all electron mass terms. Appendix A summarizes higher-order perturbative QCD corrections to heavy-quark loops that are discussed in Sec. III B. Appendix L displays fluxaveraged spectra in experimental conditions of DUNE, MINERvA, NOvA, and T2K experiments.

\section{NEUTRINO-ELECTRON SCATTERING}

We begin in Sec. II A by reviewing the kinematics of neutrino scattering on atomic electrons. Throughout this section we consider general charged leptons $\ell$, but in the following sections we specialize to the phenomenologically

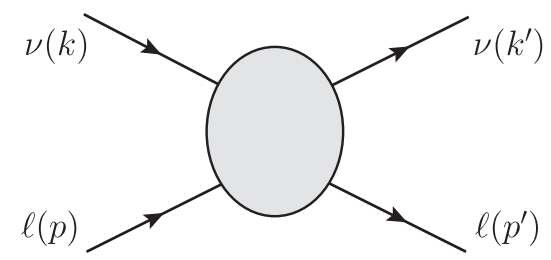

FIG. 1. Neutrino-electron scattering kinematics.

most relevant case of the electron, $\ell=e$. We introduce the relevant basis of four-fermion effective operators in Sec. II B and discuss their coefficients in Sec. II C.

\section{A. Kinematics for neutrino-electron scattering}

Consider the scattering of neutrinos on atomic electrons. We neglect the atomic binding energy and momentum compared to the energy and momentum transferred in the scattering process. Consequently, the initial electron is taken to be at rest in the laboratory frame, where the kinematics is given by $p^{\mu}=(m, 0)$ (initial electron with $\left.p^{2}=m^{2}\right), p^{\prime \mu}=\left(E^{\prime}, \boldsymbol{k}-\boldsymbol{k}^{\prime}\right)$ (final charged lepton with $\left.p^{\prime 2}=m^{\prime 2}\right), \quad k^{\mu}=(\omega, \boldsymbol{k}) \quad$ (initial neutrino), and $k^{\prime \mu}=$ $\left(\omega^{\prime}, \boldsymbol{k}^{\prime}\right)$ (final neutrino); see Fig. 1. The neutrino mass scale is much lower than the electron mass and typical neutrino beam energy, and we neglect the neutrino mass $m_{\nu}$ throughout. We will let $q^{\mu}=p^{\prime \mu}-p^{\mu}$ denote the momentum transfer to the charged lepton and write $m_{e}=m$ for the electron mass.

Elastic scattering is described by two independent kinematical variables. It is convenient to introduce the invariant momentum transfer,

$$
q^{2}=\left(p^{\prime}-p\right)^{2},
$$

and the squared energy in the center-of-mass reference frame,

$$
s=(p+k)^{2} .
$$

Note that production of heavier charged leptons in neutrino-electron scattering is possible when the neutrino beam energy is high enough. Using $s=m^{2}+2 m \omega \geq m^{\prime 2}$ we see that $\omega \geq\left(m_{\mu}^{2}-m^{2}\right) /(2 m) \approx 10.9 \mathrm{GeV}$ to produce a muon $\left(m^{\prime}=m_{\mu}\right)$, while $\omega \geq 3089 \mathrm{GeV}$ for the production of $\tau\left(m^{\prime}=m_{\tau}\right)$.

The neutrino scattering angle in the laboratory frame, $\Theta_{\nu}$, can be expressed in terms of the final neutrino energy $\omega^{\prime}$ as

$$
\begin{aligned}
\cos \Theta_{\nu} & =\frac{\omega \omega^{\prime}-m\left(\omega-\omega^{\prime}\right)-\frac{m^{2}-m^{\prime 2}}{2}}{|\mathbf{k}|\left|\mathbf{k}^{\prime}\right|} \\
& =1+\frac{m}{\omega}-\frac{m}{\omega^{\prime}}-\frac{m^{2}-m^{\prime 2}}{2 \omega \omega^{\prime}} .
\end{aligned}
$$

The final neutrino energy varies between backward and forward scattering in the range 


$$
\frac{m \omega}{m+2 \omega}+\frac{m^{2}-m^{\prime 2}}{2(m+2 \omega)} \leq \omega^{\prime} \leq \omega+\frac{m^{2}-m^{\prime 2}}{2 m}
$$

corresponding to the charged lepton energy range

$$
m+\frac{m^{\prime 2}-m^{2}}{2 m} \leq E^{\prime} \leq m+\frac{2 \omega^{2}}{m+2 \omega}+\frac{m^{\prime 2}-m^{2}}{2(m+2 \omega)} \text {. }
$$

The angle between recoil charged lepton direction and the neutrino beam direction, $\Theta_{e}$, is given by

$$
\cos \Theta_{e}=\frac{\omega E^{\prime}-m^{2}-m\left(\omega-E^{\prime}\right)+\frac{m^{2}-m^{\prime 2}}{2}}{\omega\left|\mathbf{p}^{\prime}\right|},
$$

and scattering is possible only in the forward cone bounded by $\Theta_{e}^{\max }$,

$$
\cos \Theta_{e}^{\max }=\sqrt{\frac{m^{\prime 2}-m^{2}}{m^{\prime 2}} \frac{(2 \omega+m)^{2}-m^{\prime 2}}{4 \omega^{2}}} .
$$

The scattering angle expression simplifies for the elastic process $\left(m^{\prime}=m\right)$ to

$$
\cos \Theta_{e}=\frac{m+\omega}{\omega} \sqrt{\frac{E^{\prime}-m}{E^{\prime}+m}}
$$

when it varies between 0 and 1, i.e., the electron is scattered always into the forward hemisphere.

\section{B. Effective neutrino-charged lepton operators}

Neutrino-electron scattering is described by the exchange of weak vector bosons $W$ and $Z$ (with masses $M_{W}$ and $M_{Z}$, respectively) in the Standard Model; cf. Fig. 2 for contributing Feynman diagrams. At energies below the electroweak scale, the interactions of neutrinos and charged leptons are determined by an equivalent effective Lagrangian [57-59]. Neglecting corrections suppressed by $1 / M_{W}^{2}$, the effective Lagrangian consists of momentum-independent four-fermion operators.

At tree level, the matching onto this effective Lagrangian $\mathcal{L}_{\text {eff }}$ is readily obtained,
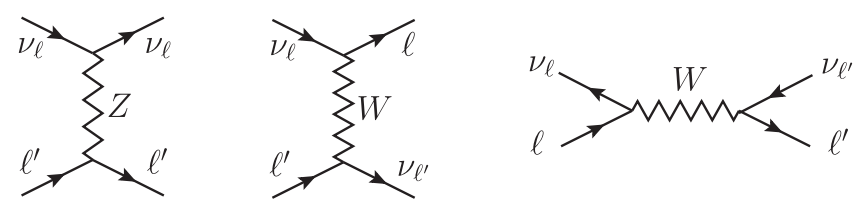

FIG. 2. Leading-order contributions to neutrino-lepton scattering in the Standard Model. The graph with the exchange of $Z$ boson contributes to the neutrino and antineutrino scattering. $\ell$ and $\ell^{\prime}$ denote charged leptons of any flavor in this figure.

$$
\mathcal{L}_{\text {eff }}=-\frac{g^{2}}{M_{W}^{2}}\left(J_{W+}\right)^{\mu}\left(J_{W^{-}}\right)_{\mu}-\frac{g^{2}}{2 M_{Z}^{2}}\left(J_{Z}\right)^{\mu}\left(J_{Z}\right)_{\mu},
$$

where $J_{W^{-}}^{\mu}, J_{W^{+}}^{\mu}=J_{W^{-}}^{\dagger \mu}$, and $J_{Z}^{\mu}$ are charged and neutral currents in the Standard Model Lagrangian coupling to $W^{+}$, $W^{-}$, and $Z$, respectively, and $g$ is the electroweak $\mathrm{SU}(2)_{\mathrm{L}}$ coupling constant. Focusing on leptonic vs quark operators, we have

$$
\begin{gathered}
J_{W^{-}}^{\mu}=\frac{1}{\sqrt{2}} \sum_{\ell} \bar{\ell} \gamma^{\mu} \mathrm{P}_{\mathrm{L}} \nu_{\ell}, \\
J_{Z}^{\mu}=\frac{1}{\cos \theta_{W}} \sum_{\ell}\left[\left(-\frac{1}{2}+\sin ^{2} \theta_{W}\right) \bar{\ell} \gamma^{\mu} \mathrm{P}_{\mathrm{L}} \ell\right. \\
\left.+\sin ^{2} \theta_{W} \bar{\ell} \gamma^{\mu} \mathrm{P}_{\mathrm{R}} \ell+\frac{1}{2} \bar{\nu}_{\ell} \gamma^{\mu} \mathrm{P}_{\mathrm{L}} \nu_{\ell}\right]
\end{gathered}
$$

where $\mathrm{P}_{\mathrm{L}}=\left(1-\gamma_{5}\right) / 2$ and $\mathrm{P}_{\mathrm{R}}=\left(1+\gamma_{5}\right) / 2$ are projection operators onto left-handed and right-handed fermions and $\theta_{W}$ denotes the weak mixing angle satisfying $M_{W} / M_{Z}=$ $\cos \theta_{W}$. After Fierz rearrangement of the charged current contribution, the result may be written as

$$
\begin{aligned}
\mathcal{L}_{\text {eff }}= & -\sum_{\ell, \ell^{\prime}} \bar{\nu}_{\ell} \gamma^{\mu} \mathrm{P}_{\mathrm{L}} \nu_{\ell} \bar{\ell}^{\prime} \gamma_{\mu}\left(c_{\mathrm{L}}^{\nu} \ell^{\prime} \mathrm{P}_{\mathrm{L}}+c_{\mathrm{R}} \mathrm{P}_{\mathrm{R}}\right) \ell^{\prime} \\
& -c \sum_{\ell \neq \ell^{\prime}} \bar{\nu}_{\ell^{\prime}} \gamma^{\mu} \mathrm{P}_{\mathrm{L}} \nu_{\ell} \bar{\ell} \gamma_{\mu} \mathrm{P}_{\mathrm{L}} \ell^{\prime},
\end{aligned}
$$

with coefficients $c_{\mathrm{L}}^{\nu_{\ell} \ell^{\prime}}, c_{\mathrm{R}}$, and $c$,

$$
\begin{aligned}
c_{\mathrm{L}}^{\nu_{\ell} \ell^{\prime}} & =2 \sqrt{2} \mathrm{G}_{\mathrm{F}}\left(\sin ^{2} \theta_{W}-\frac{1}{2}+\delta_{\ell \ell^{\prime}}\right), \\
c_{\mathrm{R}} & =2 \sqrt{2} \mathrm{G}_{\mathrm{F}} \sin ^{2} \theta_{W}, \quad c=2 \sqrt{2} \mathrm{G}_{\mathrm{F}},
\end{aligned}
$$

where we have introduced the Fermi constant $\mathrm{G}_{\mathrm{F}}=$ $g^{2} /\left(4 \sqrt{2} M_{W}^{2}\right)$, and where the Kronecker symbol $\delta_{\ell \ell^{\prime}}$ satisfies $\delta_{\ell \ell^{\prime}}=1$ for $\ell=\ell^{\prime}$ and $\delta_{\ell \ell^{\prime}}=0$ for $\ell \neq \ell^{\prime}$. Note that coefficients $c$ and $c_{\mathrm{R}}$ are the same for all combinations of lepton flavors, while the coefficient $c_{\mathrm{L}}^{\nu_{\ell} \ell^{\prime}}$ depends on whether the neutrino and charged lepton have the same flavor.

Neglecting the neutrino magnetic moment contribution [60-66], the leading-order cross section of neutrino-lepton scattering can be expressed, in all possible cases, as $[24,25,29,67-92]$

$$
\begin{aligned}
& \frac{\mathrm{d} \sigma_{\mathrm{LO}}^{\nu_{\ell} \ell^{\prime} \rightarrow \nu_{\ell} \ell^{\prime}}}{\mathrm{d} \omega^{\prime}}=\frac{m}{4 \pi}\left[\left(c_{\mathrm{L}}^{\nu_{\ell} \ell^{\prime}}\right)^{2} \mathrm{I}_{\mathrm{L}}+c_{\mathrm{R}}^{2} \mathrm{I}_{\mathrm{R}}+c_{\mathrm{L}}^{\nu_{\ell} \ell^{\prime}} c_{\mathrm{R}} \mathrm{I}_{\mathrm{R}}^{\mathrm{L}}\right], \\
& \frac{\mathrm{d} \sigma_{\mathrm{LO}}^{\overline{\nu_{\ell}} \ell^{\prime} \rightarrow \bar{\nu}_{\ell} \ell^{\prime}}}{\mathrm{d} \omega^{\prime}}=\frac{m}{4 \pi}\left[\left(c_{\mathrm{L}}^{\nu_{\ell} \ell^{\prime}}\right)^{2} \mathrm{I}_{\mathrm{R}}+c_{\mathrm{R}}^{2} \mathrm{I}_{\mathrm{L}}+c_{\mathrm{L}}^{\nu_{\ell} \ell^{\prime}} c_{\mathrm{R}} \mathrm{I}_{\mathrm{R}}^{\mathrm{L}}\right],
\end{aligned}
$$


TABLE I. Effective couplings (in units $10^{-5} \mathrm{GeV}^{-2}$ ) in the Fermi theory of neutrino-fermion scattering with four quark flavors at the scale $\mu=2 \mathrm{GeV}$. The error due to the uncertainty of Standard Model parameters is added in quadrature to a perturbative error of matching.

\begin{tabular}{lccccc}
\hline \hline$c_{\mathrm{L}}^{\nu_{\ell} \ell^{\prime}}, \ell=\ell^{\prime}$ & $c_{\mathrm{L}}^{\nu_{\ell} \ell^{\prime}}, \ell \neq \ell^{\prime}$ & $c_{\mathrm{R}}$ & $c_{\mathrm{L}}^{u}$ & $c_{\mathrm{R}}^{u}$ & $c_{\mathrm{L}}^{d}$ \\
\hline \hline $2.39818(33)$ & $-0.90084(32)$ & $0.76911(60)$ & $1.14065(13)$ & $-0.51173(38)$ & $-1.41478(12)$ \\
\hline \hline & $\left.\frac{\mathrm{d} \sigma_{\mathrm{LO}}^{\nu_{\ell} \ell^{\prime} \rightarrow \nu_{\ell^{\prime} \ell}}}{\mathrm{d} \omega^{\prime}}\right|_{\ell \neq \ell^{\prime}}=\frac{m}{4 \pi} c^{2} \mathrm{I}_{\mathrm{L}}$, & $(16)$ & $\bar{\omega}=\left(\frac{c_{\mathrm{L}}^{\nu_{\ell} \ell}}{c_{\mathrm{R}}}-1\right) \frac{m}{2} \cdot 17(20)$
\end{tabular}

We discuss the impact of radiative corrections on the

$$
\left.\frac{\mathrm{d} \sigma_{\mathrm{LO}}^{\bar{\nu}_{\ell} \ell \bar{\nu}_{\ell^{\prime}} \ell^{\prime}}}{\mathrm{d} \omega^{\prime}}\right|_{\ell \neq \ell^{\prime}}=\frac{m}{4 \pi} c^{2} \mathrm{I}_{\mathrm{R}}
$$

with kinematical factors:

$$
\begin{gathered}
\mathrm{I}_{\mathrm{L}}=\frac{(k \cdot p)\left(k^{\prime} \cdot p^{\prime}\right)}{m^{2} \omega^{2}}=1+\frac{m^{2}-m^{\prime 2}}{2 m \omega} \rightarrow 1, \\
\mathrm{I}_{\mathrm{R}}=\frac{\left(k \cdot p^{\prime}\right)\left(k^{\prime} \cdot p\right)}{m^{2} \omega^{2}}=\frac{\omega^{\prime 2}}{\omega^{2}}\left(1+\frac{m^{\prime 2}-m^{2}}{2 m \omega^{\prime}}\right) \rightarrow \frac{\omega^{\prime 2}}{\omega^{2}}, \\
\mathrm{I}_{\mathrm{R}}^{\mathrm{L}}=-\frac{m m^{\prime}\left(k \cdot k^{\prime}\right)}{m^{2} \omega^{2}}=-\frac{m^{\prime}}{\omega}\left(1-\frac{\omega^{\prime}}{\omega}+\frac{m^{2}-m^{\prime 2}}{2 m \omega}\right) \\
\rightarrow-\frac{m}{\omega}\left(1-\frac{\omega^{\prime}}{\omega}\right),
\end{gathered}
$$

where the limit of elastic process, i.e., $m^{\prime}=m$, is presented in the last step. The neutrino-energy spectra in Eqs. (14)(17) are equivalent to the recoil electron energy spectra due to energy conservation: $m+\omega=E^{\prime}+\omega^{\prime}$. In particular, $\mathrm{d} \sigma / \mathrm{d} E^{\prime}=\mathrm{d} \sigma / \mathrm{d} \omega^{\prime}$. We later apply this observation to compute differential cross sections with respect to total electromagnetic energy in the presence of radiative corrections. To study the angular spectrum, the differential cross section can be obtained by exploiting

$$
\mathrm{d} E^{\prime}=\frac{4 m \omega^{2}(m+\omega)^{2} \cos \Theta_{e} \mathrm{~d} \cos \Theta_{e}}{\left[(m+\omega)^{2}-\omega^{2} \cos ^{2} \Theta_{e}\right]^{2}} .
$$

We observe that the contribution from the interference term $\mathrm{I}_{\mathrm{R}}^{\mathrm{L}}$ is suppressed by the charged lepton mass. The neutrino and antineutrino scattering are related by the substitution $\mathrm{I}_{\mathrm{L}} \leftrightarrow \mathrm{I}_{\mathrm{R}}\left(k \leftrightarrow k^{\prime}\right)$ or equivalently $c_{\mathrm{L}}^{\nu_{\ell} \ell^{\prime}} \leftrightarrow c_{\mathrm{R}}$.

Note that $\nu_{\ell} \ell \rightarrow \nu_{\ell} \ell$ and $\bar{\nu}_{\ell} \ell \rightarrow \bar{\nu}_{\ell} \ell$ cross sections involving one flavor seem to be not positive definite for energies comparable with the charged lepton mass due to the helicity-flip interference term $c_{\mathrm{L}}^{\nu_{\ell} \ell} c_{\mathrm{R}}$. However, the cross section is always positive in the physical region of scattering $m \omega /(m+2 \omega)<\omega^{\prime}<\omega$ and can vanish only in the case of forward recoil electrons with maximum energy $E^{\prime}=m+2 \omega^{2} /(m+2 \omega)$ [93-96] in the scattering of an electron antineutrino of energy $\bar{\omega}$ : cancellation (22) in Sec. V D.

\section{Effective neutrino-lepton and neutrino-quark interactions beyond leading order}

Higher-order electroweak and QCD contributions modify couplings in the effective Lagrangian of Eq. (12). The evaluation of virtual NLO corrections to elastic neutrinocharged lepton scattering also involves interaction with quarks and gluons; see Secs. III B and III C. The relevant neutral current part of the effective neutrino-quark Lagrangian is

$$
\mathcal{L}_{\text {eff }}^{q}=-\sum_{\ell, q} \bar{\nu}_{\ell} \gamma^{\mu} \mathrm{P}_{\mathrm{L}} \nu_{\ell} \bar{q} \gamma_{\mu}\left(c_{\mathrm{L}}^{q} \mathrm{P}_{\mathrm{L}}+c_{\mathrm{R}}^{q} \mathrm{P}_{\mathrm{R}}\right) q,
$$

with (neutrino flavor independent) left- and right-handed couplings $c_{\mathrm{L}}^{q}$ and $c_{\mathrm{R}}^{q}$, respectively. At tree level,

$c_{\mathrm{L}}^{q}=2 \sqrt{2} \mathrm{G}_{\mathrm{F}}\left(T_{q}^{3}-Q_{q} \sin ^{2} \theta_{W}\right), \quad c_{\mathrm{R}}^{q}=-2 \sqrt{2} \mathrm{G}_{\mathrm{F}} Q_{q} \sin ^{2} \theta_{W}$,

where $T_{q}^{3}$ denotes the quark isospin $(+1 / 2$ for $q=u, c$, $-1 / 2$ for $q=d, s)$ and $Q_{q}$ its electric charge in units of the positron charge $(+2 / 3$ for $q=u, c,-1 / 3$ for $q=d, s)$. For numerical analysis, we employ low-energy effective couplings from Ref. [47]. For definiteness, we take inputs in four-flavor QCD $\left(n_{f}=4\right)$ at renormalization scale $\mu=2 \mathrm{GeV}$ in the $\overline{\mathrm{MS}}$ scheme and do not distinguish between couplings to $u(d)$ and $c(s)$ quarks. ${ }^{2}$

The effective Lagrangians of Eqs. (12) and (23), and the corresponding charged current quark operators [47], determine neutrino scattering rates at $\mathrm{GeV}$ energy scales, up to corrections suppressed by powers of electroweak scale particle masses. Electroweak scale physics is encoded in

\footnotetext{
${ }^{2}$ In Ref. [47], one-loop matching to the Standard Model is performed at the electroweak scale accounting for the leading QCD corrections with one exchanged gluon inside quark loops and neglecting masses of all fermions except the top quark compared to the electroweak scale. The matching is accompanied by renormalization group evolution to $\mathrm{GeV}$ scales to resum large electroweak logarithms in the effective couplings. The relation of the couplings in Table I to various definitions of $\mathrm{G}_{\mathrm{F}}$ and $\sin ^{2} \theta_{W}$ is discussed in Ref. [47].
} 

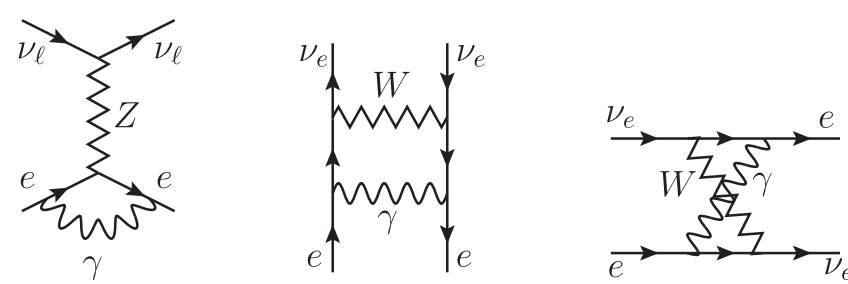

FIG. 3. Virtual corrections to elastic neutrino-electron scattering in the Standard Model corresponding to the vertex correction in effective theory.

the values of the operator coefficients, summarized in Table I. Real photon radiation and virtual corrections involving the photon and other light particles must still be calculated within the effective theory.

\section{VIRTUAL QED CORRECTIONS}

In this section, we present virtual corrections, considering QED vertex corrections involving virtual photons in Sec. III A and closed fermion loop contributions from leptons and heavy quarks in Sec. III B. We estimate the correction coming from light-quark loops in Sec. III C.

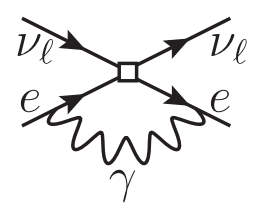

FIG. 4. QED vertex correction to elastic neutrino-electron scattering in effective theory.

\section{A. QED vertex correction}

We consider one-loop virtual corrections in elastic (anti)neutrino-electron scattering $\nu_{\ell} e \rightarrow \nu_{\ell} e\left(\bar{\nu}_{\ell} e \rightarrow \bar{\nu}_{\ell} e\right)$. Within the Standard Model, the vertex correction is given by the diagrams in Fig. 3, while only the single diagram in Fig. 4 contributes in the effective theory. The usual field renormalization factors must be applied to external legs.

First, we evaluate the one-loop vertex correction to the matrix element of left-handed (L) and right-handed (R) charged lepton currents $\mathrm{J}_{\mu}^{\mathrm{L}, \mathrm{R}}=\bar{e}\left(p^{\prime}\right) \gamma_{\mu} \mathrm{P}_{\mathrm{L}, \mathrm{R}} e(p)$ from Eq. (12). We perform the integration in $d=4-2 \varepsilon$ dimensions of spacetime to regularize the ultraviolet divergence,

$$
\delta \mathbf{J}_{\mu}^{\mathrm{L}, \mathrm{R}}=-e^{2} \int \frac{i \mathrm{~d}^{d} L}{(2 \pi)^{d}} \frac{\bar{e}\left(p^{\prime}\right) \gamma^{\lambda}\left(\not p^{\prime}-\not L+m\right) \gamma_{\mu} \mathrm{P}_{\mathrm{L}, \mathrm{R}}(\not p-\not L+m) \gamma^{\rho} e(p)}{\left(L^{2}-\lambda^{2}\right)\left((p-L)^{2}-m^{2}\right)\left(\left(p^{\prime}-L\right)^{2}-m^{2}\right)}\left(g_{\lambda \rho}-\left(1-\xi_{\gamma}\right) \frac{L_{\lambda} L_{\rho}}{L^{2}-a \xi_{\gamma} \lambda^{2}}\right),
$$

where $\not k \equiv k_{\mu} \gamma^{\mu}$ for any four-vector $k, \xi_{\gamma}$ is the photon gauge parameter, and $a$ is an arbitrary constant associated with the photon mass regulator. The small photon mass $\lambda$ is introduced to regulate infrared (IR) divergences. The corresponding field renormalization factor of external charged leptons is

$$
Z_{\ell}=1-\frac{\alpha}{4 \pi} \frac{\xi_{\gamma}}{\varepsilon}-\frac{\alpha}{4 \pi}\left(\ln \frac{\mu^{2}}{m^{2}}+2 \ln \frac{\lambda^{2}}{m^{2}}+4\right)+\frac{\alpha}{4 \pi}\left(1-\xi_{\gamma}\right)\left(\ln \frac{\mu^{2}}{\lambda^{2}}+1+\frac{a \xi_{\gamma} \ln a \xi_{\gamma}}{1-a \xi_{\gamma}}\right) .
$$

Neglecting Lorentz structures whose contractions with the neutrino current vanish at $m_{\nu}=0$, the resulting correction can be expressed as ${ }^{3}$

$$
\left(Z_{\ell}-1\right) \mathbf{J}_{\mu}^{\mathrm{L}, \mathrm{R}}+\delta \mathbf{J}_{\mu}^{\mathrm{L}, \mathrm{R}}=\frac{\alpha}{\pi}\left(f_{1} \mathbf{J}_{\mu}^{\mathrm{L}, \mathrm{R}}+f_{2} \mathrm{j}_{\mu}^{\mathrm{L}, \mathrm{R}}\right),
$$

in terms of form factors $f_{1}$ and $f_{2}$, and the additional currents $\mathrm{j}_{\mu}^{\mathrm{L}}$ and $\mathrm{j}_{\mu}^{\mathrm{R}}$ :

\footnotetext{
${ }^{3}$ Note that the vertex correction can be expressed as a modification of vector and axial currents:

$$
\begin{gathered}
\bar{e}\left(p^{\prime}\right) \gamma_{\mu} e(p) \rightarrow \bar{e}\left(p^{\prime}\right) \gamma_{\mu} e(p)+\frac{\alpha}{\pi} \bar{e}\left(p^{\prime}\right)\left(f_{1} \gamma_{\mu}+f_{2} \frac{i \sigma_{\mu \nu} q^{\nu}}{2 m}\right) e(p), \\
\bar{e}\left(p^{\prime}\right) \gamma_{\mu} \gamma_{5} e(p) \rightarrow \bar{e}\left(p^{\prime}\right) \gamma_{\mu} \gamma_{5} e(p)+\frac{\alpha}{\pi}\left(f_{1}-f_{2}\right) \bar{e}\left(p^{\prime}\right) \gamma_{\mu} \gamma_{5} e(p) .
\end{gathered}
$$

$$
\begin{aligned}
& \mathrm{j}_{\mu}^{\mathrm{L}}=\frac{1}{2} \bar{e}\left(p^{\prime}\right)\left(\gamma_{\mu} \gamma_{5}+\frac{i \sigma_{\mu \nu} q^{\nu}}{2 m}\right) e(p), \\
& \mathrm{j}_{\mu}^{\mathrm{R}}=\frac{1}{2} \bar{e}\left(p^{\prime}\right)\left(-\gamma_{\mu} \gamma_{5}+\frac{i \sigma_{\mu \nu} q^{\nu}}{2 m}\right) e(p) .
\end{aligned}
$$

Here $\sigma_{\mu \nu}=\frac{i}{2}\left[\gamma_{\mu}, \gamma_{\nu}\right]$.

Using Eqs. (25) and (26), the UV finite and gaugeindependent virtual correction is given in Eq. (29) by oneloop QED form factors [97,98]:

$$
\begin{aligned}
f_{1}(\beta)= & -\frac{1}{2 \beta}\left(\beta-\frac{1}{2} \ln \frac{1+\beta}{1-\beta}\right) \ln \frac{\lambda^{2}}{m^{2}} \\
& +\frac{1}{\beta}\left[\frac{3+\rho}{8} \ln \frac{1+\beta}{1-\beta}-\frac{1}{8} \ln \frac{1+\beta}{1-\beta} \ln \left(2 \frac{1+\rho}{\rho}\right)\right] \\
& -\frac{1}{2 \beta}\left(\operatorname{Li}_{2} \frac{\beta-1+\rho}{2 \beta}-\mathrm{Li}_{2} \frac{\beta+1-\rho}{2 \beta}\right)-1,
\end{aligned}
$$




$$
f_{2}(\beta)=\frac{\rho}{4 \beta} \ln \frac{1+\beta}{1-\beta},
$$

which are expressed in terms of the recoil electron velocity $\beta$ and the parameter $\rho$ :

$$
\beta=\sqrt{1-\frac{m^{2}}{E^{\prime 2}}}, \quad \rho=\sqrt{1-\beta^{2}}=\frac{m}{E^{\prime}} .
$$

The vertex correction (29) to the unpolarized cross section can be expressed as a sum of factorizable and nonfactorizable terms:

$$
\mathrm{d} \sigma_{v}^{\nu_{\ell} e \rightarrow \nu_{\ell} e}=\frac{\alpha}{\pi} \delta_{v} \mathrm{~d} \sigma_{\mathrm{LO}}^{\nu_{\ell} e \rightarrow \nu_{\ell} e}+\mathrm{d} \sigma_{v, \mathrm{NF}}^{\nu_{\ell} e \rightarrow \nu_{\ell} e} .
$$

The factorizable correction is given by

$$
\delta_{v}=2 f_{1} .
$$

The nonfactorizable term $\mathrm{d} \sigma_{v, \mathrm{NF}}^{\nu_{\ell} \boldsymbol{\nu}_{\ell} e}$ is obtained by modifying kinematical factors $\mathrm{I}_{i}$ in Eqs. (14) and (15) as $\mathrm{I}_{i} \rightarrow$ $\mathrm{I}_{i}+\frac{\alpha}{\pi} f_{2} \delta^{v} \mathrm{I}_{i}$ where

$$
\begin{gathered}
\delta^{v} \mathrm{I}_{\mathrm{L}}=\delta^{v} \mathrm{I}_{\mathrm{R}}=\frac{1}{2} \mathrm{I}_{\mathrm{R}}^{\mathrm{L}}-\frac{\omega^{\prime}}{\omega}, \\
\delta^{v} \mathrm{I}_{\mathrm{R}}^{\mathrm{L}}=2\left(\mathrm{I}_{\mathrm{L}}+\mathrm{I}_{\mathrm{R}}-\frac{\omega^{\prime}}{\omega}\right)-\mathrm{I}_{\mathrm{R}}^{\mathrm{L}} .
\end{gathered}
$$

The resulting vertex correction to the unpolarized cross section of Eq. (35) is in agreement with Refs. [29,37]. In the limit of a massless electron, the Pauli form factor vanishes, $f_{2}(\beta) \rightarrow 0$, and the correction becomes exactly factorizable.

\section{B. Closed fermion loops: Leptons and heavy quarks}

In addition to the corrections involving virtual photons in Sec. III A, we must account for the corrections with a closed fermion loop of Fig. 5. These corrections correspond to the diagram of penguin type and the effects of $\gamma-Z$ mixing in the Standard Model; cf. Fig. 6. They represent the EFT determination of the kinematical dependence of electroweak corrections; cf. Refs. [25,27].

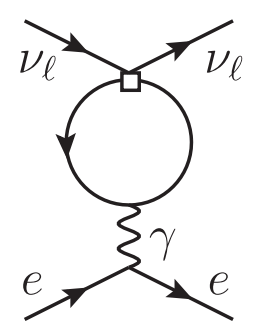

FIG. 5. Long-range dynamics in elastic neutrino-electron scattering in the effective theory. Loops with all interacting fields in the theory are summed up.
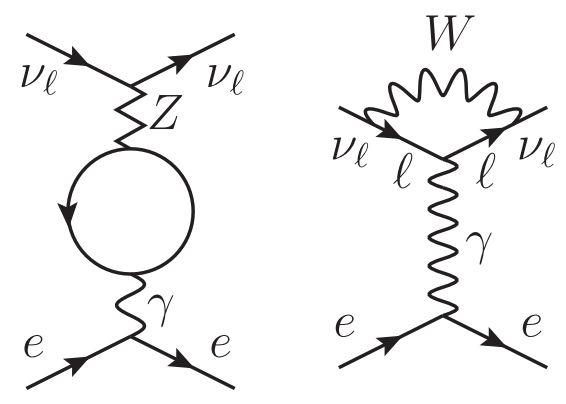

FIG. 6. Standard Model diagrams giving rise to long-range dynamics in EFT: $\gamma-Z$ mixing and penguin-type diagram.

In this section, we consider the loop contribution from an arbitrary fermion with mass $m_{f}$ and charge $Q_{f}$ (in units of the positive positron charge) and effective left- and righthanded couplings $c_{\mathrm{L}}^{f}$ and $c_{\mathrm{R}}^{f}$, respectively, as in Eqs. (12) and (23). Note that the coupling $c_{\mathrm{L}}^{f}$ for charged leptons $(f=\ell)$ depends on the neutrino flavor. This perturbative treatment applies to loops involving charged leptons or heavy quarks $\left(m_{f} \gg \Lambda_{\mathrm{QCD}}\right)$. Light quarks require a nonperturbative treatment, as discussed in Sec. III C below. Starting from the $n_{f}=4$ flavor theory discussed in Sec. II C, we treat the charm quark as heavy and the up, down, and strange quarks as light.

The correction can be expressed as a modification of electron left- and right-handed currents, $c_{\mathrm{L}, \mathrm{R}} \mathrm{J}_{\mu}^{\mathrm{L}, \mathrm{R}} \rightarrow$ $c_{\mathrm{L}, \mathrm{R}} \mathrm{J}_{\mu}^{\mathrm{L}, \mathrm{R}}+c_{\mathrm{L}, \mathrm{R}}^{f} \delta \mathbf{J}_{\mu}^{\mathrm{L}, \mathrm{R}}$,

$$
\begin{aligned}
\delta \mathrm{J}_{\mu}^{\mathrm{L}, \mathrm{R}}= & Q_{f} e^{2} \bar{e}\left(p^{\prime}\right) \gamma^{\lambda} e(p) \frac{-g_{\lambda \rho}}{q^{2}} \\
& \times \int \frac{i \mathrm{~d}^{d} L}{(2 \pi)^{d}} \frac{\operatorname{Tr}\left[\gamma^{\rho}\left(\not L+m_{f}\right) \gamma_{\mu} \mathrm{P}_{\mathrm{L}, \mathrm{R}}\left(\not L-\not 1+m_{f}\right)\right]}{\left(L^{2}-m_{f}^{2}\right)\left((L-q)^{2}-m_{f}^{2}\right)},
\end{aligned}
$$

and does not depend on the photon gauge. Corrections to either left- or right-handed currents are vectorlike and may be written

$$
\delta \mathbf{J}_{\mu}^{\mathrm{L}}=\delta \mathbf{J}_{\mu}^{\mathrm{R}}=Q_{f} \frac{\alpha}{2 \pi} \Pi\left(q^{2}, m_{f}\right)\left(\mathbf{J}_{\mu}^{\mathrm{L}}+\mathbf{J}_{\mu}^{\mathrm{R}}\right) .
$$

At renormalization scale $\mu$ in the $\overline{\mathrm{MS}}$ scheme, the form factor $\Pi$ is

$$
\begin{aligned}
\Pi\left(q^{2}, m_{f}\right)= & \frac{1}{3} \ln \frac{\mu^{2}}{m_{f}^{2}}+\frac{5}{9}+\frac{4 m_{f}^{2}}{3 q^{2}}+\frac{1}{3}\left(1+\frac{2 m_{f}^{2}}{q^{2}}\right) \\
& \times \sqrt{1-\frac{4 m_{f}^{2}}{q^{2}}} \ln \frac{\sqrt{1-\frac{4 m_{f}^{2}}{q^{2}}}-1}{\sqrt{1-\frac{4 m_{f}^{2}}{q^{2}}}+1},
\end{aligned}
$$

and corresponds to vacuum polarization in QED [99-103]. 
The resulting "dynamical" correction to the unpolarized cross section, $\mathrm{d} \sigma_{\mathrm{dyn}}^{\nu_{e} e \nu_{\ell} e}$, can be expressed in the following form:

$$
\mathrm{d} \sigma_{\mathrm{dyn}}^{\nu_{e} e \rightarrow \nu_{e} e}=\frac{\alpha}{\pi} \sum_{f \neq u d s} Q_{f} \Pi\left(q^{2}, m_{f}\right) \mathrm{d} \tilde{\sigma}_{\mathrm{dyn}, \mathrm{f}}^{\nu_{\ell} e \rightarrow \nu_{\ell} e}+\mathrm{d} \sigma_{\mathrm{dyn}, u d s}^{\nu_{\ell} e \rightarrow \nu_{e} e} .
$$

The contribution from three light flavors $\mathrm{d} \sigma_{\mathrm{dyn}, u d s}^{\nu_{\ell} e \rightarrow \nu_{\ell} e}$ is discussed below in Sec. III C. The reduced cross section $\mathrm{d} \tilde{\sigma}_{\mathrm{dyn}, \mathrm{f}}^{\nu_{\ell} e \nu_{\ell} e}$ is obtained by replacing $\nu_{\ell} e$ couplings in Eqs. (14) and (15) as

$$
\begin{gathered}
\left(c_{\mathrm{L}}^{\nu_{\ell} \ell^{\prime}}\right)^{2} \rightarrow c_{\mathrm{L}}^{\nu_{\ell} \ell^{\prime}}\left(c_{\mathrm{L}}^{f}+c_{\mathrm{R}}^{f}\right), \\
\left(c_{\mathrm{R}}\right)^{2} \rightarrow c_{\mathrm{R}}\left(c_{\mathrm{L}}^{f}+c_{\mathrm{R}}^{f}\right), \\
c_{\mathrm{L}}^{\nu_{\ell} \ell^{\prime}} c_{\mathrm{R}} \rightarrow \frac{1}{2}\left(c_{\mathrm{L}}^{\nu_{\ell} \ell^{\prime}}+c_{\mathrm{R}}\right)\left(c_{\mathrm{L}}^{f}+c_{\mathrm{R}}^{f}\right) .
\end{gathered}
$$

The sum in Eq. (42) extends over all charged leptons $(e, \mu, \tau)$ and heavy quarks $(c)$ in the theory (a factor $N_{c}=3$ is obtained in the sum over colors for heavy quarks). We also include QCD corrections due to exchanged gluons inside the quark loop; see Refs. [104-107] and Appendix A for exact expressions.

The momentum transfer in elastic neutrino-electron scattering is suppressed by the electron mass,

$$
0 \leq-q^{2}<2 m \omega .
$$

For neutrino beam energies smaller than $10 \mathrm{GeV}$, this implies $\left|q^{2}\right| \lesssim 0.01 \mathrm{GeV}^{2}$. Consequently, the contribution of loops with heavy quarks can be well approximated substituting $\Pi\left(q^{2}, m_{f}\right) \rightarrow \Pi\left(0, m_{f}\right)$.

\section{Light-quark contribution}

At small $q^{2}$, QCD perturbation theory cannot be applied to evaluate the light-quark contribution in Fig. 5. We instead evaluate this contribution by relating it to measured experimental quantities.

For $\mathrm{GeV}$ energy neutrino beams, momenta in the range (46) are small compared to hadronic mass scales, and we thus evaluate the relevant hadronic tensor at $q^{2}=0$. Neglecting NLO electroweak corrections to the quark coefficients of Eq. (23), the light-quark contribution in Eq. (42) may be written as

$$
\mathrm{d} \sigma_{\mathrm{dyn}, u d s}^{\nu_{\ell} e \rightarrow \nu_{\ell} e}=\frac{\alpha}{\pi}\left(\hat{\Pi}_{3 \gamma}^{(3)}(0)-2 \sin ^{2} \theta_{W} \hat{\Pi}_{\gamma \gamma}^{(3)}(0)\right) \mathrm{d} \tilde{\sigma}_{\mathrm{dyn}, u d s}^{\nu_{\ell} e \rightarrow \nu_{\ell} e} .
$$

The reduced cross section $\mathrm{d} \tilde{\sigma}_{\mathrm{dyn}, u d s}^{\nu_{\ell} e \rightarrow \nu_{\ell} e}$ is obtained replacing $\nu_{\ell} e$ couplings in Eqs. (14) and (15) as

$$
\begin{aligned}
\left(c_{\mathrm{L}}^{\nu_{\ell} \ell^{\prime}}\right)^{2} & \rightarrow 2 \sqrt{2} \mathrm{G}_{\mathrm{F}} c_{\mathrm{L}}^{\nu_{\ell} \ell^{\prime}}, \quad c_{\mathrm{R}}^{2} \rightarrow 2 \sqrt{2} \mathrm{G}_{\mathrm{F}} c_{\mathrm{R}}, \\
c_{\mathrm{L}}^{\nu_{\ell} \ell^{\prime}} c_{\mathrm{R}} & \rightarrow \sqrt{2} \mathrm{G}_{\mathrm{F}}\left(c_{\mathrm{L}}^{\nu_{\ell} \ell^{\prime}}+c_{\mathrm{R}}\right) .
\end{aligned}
$$

The quantity $\Pi_{\gamma \gamma}$ is defined by the vacuum correlation function,

$$
\begin{aligned}
& \left(q^{\mu} q^{\nu}-q^{2} g^{\mu \nu}\right) \Pi_{\gamma \gamma}\left(q^{2}\right) \\
& \quad=4 i \pi^{2} \int \mathrm{d}^{d} x e^{i q \cdot x}\left\langle 0\left|T\left\{J_{\gamma}^{\mu}(x) J_{\gamma}^{\nu}(0)\right\}\right| 0\right\rangle,
\end{aligned}
$$

where $J_{\gamma}^{\mu}=\sum_{q} Q_{q} \bar{q} \gamma^{\mu} q$ is the quark electromagnetic current. Similarly, $\Pi_{3 \gamma}$ is given by

$$
\begin{aligned}
& \left(q^{\mu} q^{\nu}-q^{2} g^{\mu \nu}\right) \Pi_{3 \gamma}\left(q^{2}\right) \\
& \quad=4 i \pi^{2} \int \mathrm{d}^{d} x e^{i q \cdot x}\left\langle 0\left|T\left\{J_{3}^{\mu}(x) J_{\gamma}^{\nu}(0)\right\}\right| 0\right\rangle
\end{aligned}
$$

where $J_{3}^{\mu}=\sum_{q} T_{q}^{3} \bar{q} \gamma^{\mu} q$ is (the third component of) the quark isospin current. The current-current correlation functions $\hat{\Pi}_{i j}^{(3)}(0)$ are evaluated at $q^{2}=0$ for $n_{f}=3$ flavors, in the $\overline{\mathrm{MS}}$ scheme.

Unlike the light-quark contribution to the photon propagator, involving only $\hat{\Pi}_{\gamma \gamma}$, the correction to neutral current neutrino-electron scattering involves also $\hat{\Pi}_{3 \gamma}$ and cannot be directly related to the total hadron production cross section in $e^{+} e^{-}$collisions. However, an approximate relation between $\hat{\Pi}_{\gamma \gamma}^{(3)}$ and $\hat{\Pi}_{3 \gamma}^{(3)}$ holds in the limit of $\mathrm{SU}(3)_{f}$ flavor symmetry for three light quarks [108,109]. In general, the flavor sums read

$$
\begin{aligned}
\hat{\Pi}_{\gamma \gamma}^{(3)}= & \sum_{i, j} Q_{i} Q_{j} \Pi^{i j}=\frac{4}{9} \Pi^{u u}+\frac{1}{9} \Pi^{d d}+\frac{1}{9} \Pi^{s s}-\frac{4}{9} \Pi^{u d} \\
& -\frac{4}{9} \Pi^{u s}+\frac{2}{9} \Pi^{d s}
\end{aligned}
$$

$$
\begin{aligned}
\hat{\Pi}_{3 \gamma}^{(3)}= & \sum_{i, j} T_{i}^{3} Q_{j} \Pi^{i j}=\frac{1}{2}\left(\frac{2}{3} \Pi^{u u}+\frac{1}{3} \Pi^{d d}+\frac{1}{3} \Pi^{s s}-\Pi^{u d}\right. \\
& \left.-\Pi^{u s}+\frac{2}{3} \Pi^{d s}\right)
\end{aligned}
$$

$\mathrm{SU}(3)_{f}$ symmetry implies $\Pi^{u u}=\Pi^{d d}=\Pi^{s s}$ and $\Pi^{u d}=$ $\Pi^{u s}=\Pi^{d s}$, and consequently, the simple relation [108] $\hat{\Pi}_{3 \gamma}^{(3)}(0) \approx \hat{\Pi}_{\gamma \gamma}^{(3)}(0)$. This allows us to express the entire light-quark contribution to the unpolarized cross section $\mathrm{d} \sigma_{\mathrm{uds}}^{\nu_{\ell} e \rightarrow \nu_{\ell} e}$ in terms of the single observable $\hat{\Pi}_{\gamma \gamma}^{(3)}(0)$.

For numerical evaluation, we use the dispersive analysis of $e^{+} e^{-}$cross-section data and measurements of hadronic $\tau$ decays combined with a perturbative treatment of the highenergy contribution in Refs. [110-112], 


$$
\left.\hat{\Pi}_{\gamma \gamma}^{(3)}(0)\right|_{\mu=2 \mathrm{GeV}}=3.597(21) .
$$

To estimate uncertainty due to the $\mathrm{SU}(3)_{f}$ symmetry approximation, we may consider an alternative $\mathrm{SU}(2)_{f}$ ansatz that sets $\Pi^{u u}=\Pi^{d d}, \Pi^{s s}=0$ and neglects disconnected, Okubo, Zweig and Iizuka (OZI)-suppressed terms $\Pi^{u d}=\Pi^{u s}=\Pi^{d s}=0$. The flavor sums (51) and (52) then yield $\hat{\Pi}_{3 \gamma}^{(3)}=9 \hat{\Pi}_{\gamma \gamma}^{(3)} / 10$, only a $10 \%$ correction to the $\mathrm{SU}(3)_{f}$ symmetry limit. In the final error budget, we consider a more conservative $20 \%$ uncertainty on this relation,

$$
\hat{\Pi}_{3 \gamma}^{(3)}(0)=(1 \pm 0.2) \hat{\Pi}_{\gamma \gamma}^{(3)}(0) .
$$

Renormalization scale dependence of the light-quark contribution (47) is perturbatively calculable. For $\mu \neq 2 \mathrm{GeV}$, the additional correction corresponds with $3 \Pi\left(0, m_{f}=\right.$ $2 \mathrm{GeV}$ ) of Eq. (41) for each quark (accounting for $N_{c}=3$ quark colors).

The replacement $\Pi\left(q^{2}\right) \rightarrow \Pi(0)$ introduces an error of relative order $m \omega / m_{\rho}^{2} \lesssim 10^{-3}$ for $\omega \lesssim \mathrm{GeV}$, where we use $m_{\rho}=770 \mathrm{MeV}$ as a typical hadronic scale. This regime includes neutrinos of energy up to the $\mathrm{TeV}$ range produced at modern high-energy accelerators, and the uncertainty is contained in the error budgets (53) and (54). At much higher neutrino energies where $q^{2}$ corrections are appreciable but still in the nonperturbative domain, the same $\mathrm{SU}(3)_{f}$ approximation [at momentum transfer $q^{2} \neq 0$ in Eq. (54)] can be used to describe the light-quark contribution. $^{4}$

\section{REAL PHOTON EMISSION}

Let us consider one-photon bremsstrahlung. Section IV A provides basic expressions for this process. We then study relevant differential observables accounting for both soft and hard photons. We start with the electron energy, electron angle, and photon energy triple-differential cross section in Sec. IV B. Integrating over one energy variable, we obtain double-differential distributions in Secs. IV C and IV D. The double-differential cross section with respect to two energy variables is described in Sec. IV E. We provide the distribution with respect to the photon energy and photon angle in Sec. IV F. Integrating it over the photon angle, we provide the photon energy spectrum in Sec. IV G. Finally, we discuss the real soft-photon correction to elastic neutrino-electron scattering and present electron and electromagnetic energy spectra in Secs. IV H and IV I, respectively. We also provide the absolute scattering cross section in Sec. IV J. Throughout this section, we present all expressions in the limit of small electron mass and provide expressions for general mass in the Appendix. For the

\footnotetext{
${ }^{4}$ See Ref. [113] for a discussion of $\hat{\Pi}_{\gamma \gamma}\left(q^{2}\right)-\hat{\Pi}_{\gamma \gamma}(0)$.
}
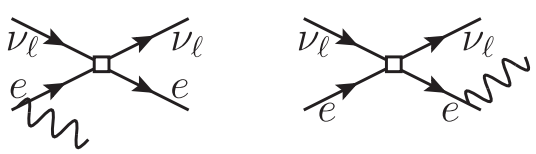

FIG. 7. One-photon bremsstrahlung in elastic neutrino-electron scattering.

energy spectra in Secs. IV H and IV I, we provide a general discussion of momentum regions at arbitrary mass, but present the massless limit and relegate details to the Appendix.

\section{A. Radiation of one photon}

The one-photon bremsstrahlung amplitude $\mathrm{T}^{1 \gamma}$ (cf. Fig. 7) contains terms corresponding to radiation from the initial electron $\mathrm{T}_{i}^{1 \gamma}$ and from the final electron $\mathrm{T}_{f}^{1 \gamma}$,

$$
\mathrm{T}^{1 \gamma}=\mathrm{T}_{i}^{1 \gamma}+\mathrm{T}_{f}^{1 \gamma}
$$

The amplitude $\mathrm{T}_{i}^{1 \gamma}$ is obtained from the tree-level amplitude with the substitution

$$
e(p) \rightarrow e \varepsilon_{\rho}^{*} \frac{\not p-\not k_{\gamma}+m}{\left(p-k_{\gamma}\right)^{2}-m^{2}} \gamma^{\rho} e(p),
$$

where $k_{\gamma}$ is a photon momentum and $\varepsilon_{\rho}^{*}$ is the photon polarization vector. The amplitude $\mathrm{T}_{f}^{1 \gamma}$ is obtained from the tree-level amplitude with the substitution

$$
\bar{e}\left(p^{\prime}\right) \rightarrow e \varepsilon_{\rho}^{*} \bar{e}\left(p^{\prime}\right) \gamma^{\rho} \frac{\not p^{\prime}+\not k_{\gamma}+m^{\prime}}{\left(p^{\prime}+k_{\gamma}\right)^{2}-m^{\prime 2}}
$$

Evaluating the spin-averaged squared matrix element, $\sum_{\text {spin }}\left|\mathrm{T}^{1 \gamma}\right|^{2}$, we obtain for the bremsstrahlung cross sections:

$$
\begin{aligned}
\mathrm{d} \sigma_{\mathrm{LO}}^{\nu_{\ell} e \rightarrow \nu_{\ell} e \gamma} & =\frac{\alpha}{4 \pi} \frac{m \omega}{\pi^{3}}\left[\left(c_{\mathrm{L}}^{\nu_{\ell} e}\right)^{2} \tilde{\mathrm{I}}_{\mathrm{L}}+c_{\mathrm{R}}^{2} \tilde{\mathrm{I}}_{\mathrm{R}}+c_{\mathrm{L}}^{\nu_{\ell} e} c_{\mathrm{R}} \tilde{\mathrm{I}}_{\mathrm{R}}^{\mathrm{L}}\right], \\
\mathrm{d} \sigma_{\mathrm{LO}}^{\bar{\nu}_{\ell} e \rightarrow \bar{\nu}_{\ell} e \gamma} & =\frac{\alpha}{4 \pi} \frac{m \omega}{\pi^{3}}\left[\left(c_{\mathrm{L}}^{\nu_{\ell} e}\right)^{2} \tilde{\mathrm{I}}_{\mathrm{R}}+c_{\mathrm{R}}^{2} \tilde{\mathrm{I}}_{\mathrm{L}}+c_{\mathrm{L}}^{\nu_{\ell} e} c_{\mathrm{R}} \tilde{\mathrm{I}}_{\mathrm{R}}^{\mathrm{L}}\right],
\end{aligned}
$$

where terms $\tilde{\mathrm{I}}_{i}$ contain the phase-space integration

$$
\tilde{\mathrm{I}}_{i}=\int \frac{R_{i}}{m^{2} \omega^{2}} \delta^{4}\left(k+p-k_{\gamma}-k^{\prime}-p^{\prime}\right) \frac{\mathrm{d}^{3} \vec{k}_{\gamma}}{2 k_{\gamma}} \frac{\mathrm{d}^{3} \vec{k}^{\prime}}{2 \omega^{\prime}} \frac{\mathrm{d}^{3} \vec{p}^{\prime}}{2 E^{\prime}},
$$

and kinematical factors $R_{i}$ are expressed in terms of particle momenta as 


$$
\begin{aligned}
& R_{\mathrm{L}}=-\mathrm{I}_{\mathrm{L}}\left[\frac{p^{\mu}}{\left(p \cdot k_{\gamma}\right)}-\frac{p^{\prime \mu}}{\left(p^{\prime} \cdot k_{\gamma}\right)}\right]^{2} m^{2} \omega^{2}+\frac{\left(k \cdot p^{\prime}\right)\left(k^{\prime} \cdot p^{\prime}\right)}{\left(k_{\gamma} \cdot p^{\prime}\right)}-\frac{(k \cdot p)\left(k^{\prime} \cdot p\right)}{\left(k_{\gamma} \cdot p\right)}+\frac{(k \cdot p)\left(k^{\prime} \cdot p^{\prime}\right)}{\left(k_{\gamma} \cdot p^{\prime}\right)}-\frac{(k \cdot p)\left(k^{\prime} \cdot p^{\prime}\right)}{\left(k_{\gamma} \cdot p\right)} \\
&+\frac{\left(k^{\prime} \cdot p^{\prime}\right)\left(k \cdot k_{\gamma}\right)}{\left(k_{\gamma} \cdot p\right)}\left(1+\frac{m^{2}}{\left(k_{\gamma} \cdot p\right)}-\frac{\left(p \cdot p^{\prime}\right)}{\left(k_{\gamma} \cdot p^{\prime}\right)}\right)+\frac{(k \cdot p)\left(k^{\prime} \cdot k_{\gamma}\right)}{\left(k_{\gamma} \cdot p^{\prime}\right)}\left(1-\frac{m^{\prime 2}}{\left(k_{\gamma} \cdot p^{\prime}\right)}+\frac{\left(p \cdot p^{\prime}\right)}{\left(k_{\gamma} \cdot p\right)}\right), \\
& R_{\mathrm{R}}=-\mathrm{I}_{\mathrm{R}}\left[\frac{p^{\mu}}{\left(p \cdot k_{\gamma}\right)}-\frac{p^{\prime \mu}}{\left(p^{\prime} \cdot k_{\gamma}\right)}\right]^{2} m^{2} \omega^{2}+\frac{\left(k \cdot p^{\prime}\right)\left(k^{\prime} \cdot p^{\prime}\right)}{\left(k_{\gamma} \cdot p^{\prime}\right)}-\frac{(k \cdot p)\left(k^{\prime} \cdot p\right)}{\left(k_{\gamma} \cdot p\right)}+\frac{\left(k^{\prime} \cdot p\right)\left(k \cdot p^{\prime}\right)}{\left(k_{\gamma} \cdot p^{\prime}\right)}-\frac{\left(k^{\prime} \cdot p\right)\left(k \cdot p^{\prime}\right)}{\left(k_{\gamma} \cdot p\right)} \\
&+\frac{\left(k \cdot p^{\prime}\right)\left(k^{\prime} \cdot k_{\gamma}\right)}{\left(k_{\gamma} \cdot p\right)}\left(1+\frac{m^{2}}{\left(k_{\gamma} \cdot p\right)}-\frac{\left(p \cdot p^{\prime}\right)}{\left(k_{\gamma} \cdot p^{\prime}\right)}\right)+\frac{\left(k^{\prime} \cdot p\right)\left(k \cdot k_{\gamma}\right)}{\left(k_{\gamma} \cdot p^{\prime}\right)}\left(1-\frac{m^{\prime 2}}{\left(k_{\gamma} \cdot p^{\prime}\right)}+\frac{\left(p \cdot p^{\prime}\right)}{\left(k_{\gamma} \cdot p\right)}\right), \\
& R_{\mathrm{R}}^{\mathrm{L}}=-\mathrm{I}_{\mathrm{R}}^{\mathrm{L}}\left[\frac{p^{\mu}}{\left(p \cdot k_{\gamma}\right)}-\frac{p^{\prime \mu}}{\left(p^{\prime} \cdot k_{\gamma}\right)}\right]^{2} m^{2} \omega^{2}-\frac{2 m m^{\prime}\left(k \cdot k_{\gamma}\right)\left(k^{\prime} \cdot k_{\gamma}\right)}{\left(p \cdot k_{\gamma}\right)\left(p^{\prime} \cdot k_{\gamma}\right)} .
\end{aligned}
$$

Kinematical factors $I_{L}, I_{R}, I_{R}^{L}$ are given in terms of momentum invariants in Eqs. (18)-(20) and are evaluated in the kinematics of $2 \rightarrow 3$ scattering. Neutrino and antineutrino scattering are related by the substitution $R_{\mathrm{L}} \leftrightarrow R_{\mathrm{R}}$ (equivalently, $k \leftrightarrow k^{\prime}$ ). The IR-divergent parts of $R_{\mathrm{L}}$ and $R_{\mathrm{R}}$ correspond to integrals $R$ and $\hat{R}$ in Ref. [25], respectively.

\section{B. Triple-differential distribution}

We evaluate the bremsstrahlung cross section using the integration technique of Ref. [22] and provide expressions for the triple-differential cross section with respect to electron angle, electron energy, and photon energy keeping all electron mass terms in Appendix B. In the limit of small electron mass, ${ }^{5}$ the result can be approximated by the following substitutions in Eqs. (58) and (59), ${ }^{6}$

$$
\begin{aligned}
& \tilde{\mathrm{I}}_{\mathrm{L}} \underset{\omega \gg m}{\longrightarrow} {\left[\frac{\left(\omega-\omega^{\prime}\right)\left(E^{\prime 2}(2-\tilde{z})^{2}+\omega^{2}\right)}{2\left|\omega-\left(\omega-\omega^{\prime}\right)(2-\tilde{z})\right|}-\frac{E^{\prime}\left(E^{\prime 4}(2-\tilde{z})^{2}+E^{\prime 2} \omega^{2}(3 \tilde{z}-5)+E^{\prime} \omega^{3}(1-\tilde{z})+\omega^{4}\right)}{2\left(\omega-E^{\prime}\right)^{3}}\right.} \\
&+\frac{E^{\prime 2} \omega^{\prime}\left(2 E^{\prime 3}(1-\tilde{z})(2-\tilde{z})+E^{\prime 2} \omega(13+2 \tilde{z}(2 \tilde{z}-7))+2 E^{\prime} \omega^{2}(4 \tilde{z}-7)+3 \omega^{3}\right)}{2\left(\omega-E^{\prime}\right)^{4}} \\
&\left.-\frac{E^{\prime 2} \omega^{\prime 2}\left(E^{\prime 3}(1-\tilde{z})(2-\tilde{z})+E^{\prime 2} \omega(8+\tilde{z}(4 \tilde{z}-11))+E^{\prime} \omega^{2}\left(\tilde{z}^{2}+\tilde{z}-4\right)+\omega^{3} \tilde{z}\right)}{2\left(\omega-E^{\prime}\right)^{5}}\right] \mathcal{D}, \\
& \tilde{\mathrm{I}}_{\mathrm{R}} \underset{\omega \gg m}{\longrightarrow} \frac{E^{\prime 2}(1-\tilde{z})^{2}+\omega^{\prime 2}}{2}\left[\frac{\omega-\omega^{\prime}}{\left|\omega-\left(\omega-\omega^{\prime}\right)(2-\tilde{z})\right|}-\frac{E^{\prime}}{\omega-E^{\prime}}\right] \mathcal{D}, \\
& \tilde{\mathrm{I}}_{\mathrm{R}}^{\mathrm{L}} \underset{\omega \gg m}{\longrightarrow} m\left[\frac{E^{\prime 2}(2-\tilde{z})(\tilde{z}-1)+E^{\prime}\left(3 \omega^{\prime}-\left(\omega+\omega^{\prime}\right) \tilde{z}\right)-\omega \omega^{\prime}}{\left|\omega-\left(\omega-\omega^{\prime}\right)(2-\tilde{z})\right|}-\frac{E^{\prime}\left(\omega-E^{\prime}(3-\tilde{z})\right)}{\omega-E^{\prime}}\right] \mathcal{D},
\end{aligned}
$$

with the phase-space factor

$$
\mathcal{D}=\frac{\pi^{2}}{\omega^{3}} \frac{\mathrm{d} \tilde{z} \mathrm{~d} E^{\prime} \mathrm{d} k_{\gamma}}{k_{\gamma}},
$$

where $\omega^{\prime}=\omega-k_{\gamma}-E^{\prime}$ and the variable $\tilde{z} \leq 1$ is introduced to emphasize the forward direction of the relativistic electron:

\footnotetext{
${ }^{5}$ In the following, we denote the limit of small electron mass compared to all other relevant energy scales as $\omega \gg m$.

${ }^{6}$ Note that suppressed terms in the lepton mass expansion of $\tilde{\mathrm{I}}_{\mathrm{L}}$ and $\tilde{\mathrm{I}}_{\mathrm{R}}$ contribute to the cross section at the same order as $\tilde{\mathrm{I}}_{\mathrm{R}}^{\mathrm{L}}$. For a consistent power counting, one has either to neglect the interference term completely or to expand $\tilde{\mathrm{I}}_{\mathrm{L}}$ and $\tilde{\mathrm{I}}_{\mathrm{R}}$ further.
}

$$
1-\cos \theta_{e} \equiv \frac{m}{\omega}(1-\tilde{z}) .
$$

Note the difference between the electron scattering angle in the elastic process $\left[\Theta_{e}\right.$ of Eq. (6)] and in the scattering process with radiation $\left(\theta_{e}\right)$. At $m \rightarrow 0$, the physical region of kinematical variables is given by

$$
0 \leq E^{\prime} \leq \omega, \quad 2-\frac{\omega}{E^{\prime}} \leq \tilde{z} \leq 1, \quad 0 \leq k_{\gamma} \leq \omega-E^{\prime} .
$$

In the vicinity of the elastic peak,

$$
\tilde{z} \rightarrow \tilde{Z}=1-\frac{\omega^{\prime}}{\omega-\omega^{\prime}},
$$


the cross section of Eqs. (64)-(66) diverges. The small mass approximation in Eqs. (64)-(66) is valid only away from this region:

$$
|\tilde{z}-\tilde{Z}| \gg \frac{m}{E^{\prime}} \frac{k_{\gamma}^{2}}{\left(E^{\prime}+k_{\gamma}\right)^{2}} \frac{\omega^{\prime}}{\omega-\omega^{\prime}} .
$$

For a correct description in the elastic peak region, and to obtain distributions (such as energy spectra) that involve integration through this region, expressions with an electron mass of Appendix B must be used.

\section{Double-differential distribution in electron energy and electron angle}

Integrating the triple-differential distribution over the photon energy $k_{\gamma}$, we obtain the double-differential cross section with respect to the recoil electron energy and electron angle. We provide the double-differential distribution in electron energy and electron angle keeping all electron mass terms in Appendix C. In the limit of small electron mass, the cross section is given by the following substitutions in Eqs. (58) and (59) ${ }^{6}$ :

$$
\tilde{\mathrm{I}}_{i} \underset{\omega \gg m}{\longrightarrow} \frac{\pi^{2}}{\omega^{2}}\left(a_{i}+b_{i} \ln \frac{m}{2 E^{\prime}}+c_{i} \ln \frac{E^{\prime}+\omega(1-z)}{\omega-E^{\prime}}+d_{i} \ln \frac{2\left(E^{\prime}-\omega\right)^{2}}{m\left(\omega z-E^{\prime}\right)}\right) \frac{\mathrm{d} z \mathrm{~d} E^{\prime}}{\omega-E^{\prime}},
$$

with the coefficients $a_{i}, b_{i}, c_{i}$, and $d_{i}$,

$$
\begin{aligned}
& a_{\mathrm{L}}=\frac{\omega^{4}\left(E^{\prime}(z(3-2(7-2 z) z)+16)-\omega(8-z(8-(7-3 z) z))\right)}{4\left(\omega-E^{\prime}\right)^{2}\left(\omega z-E^{\prime}\right)} \\
& +\frac{E^{\prime 2} \omega\left(E^{\prime 2}(4-z)+E^{\prime} \omega(2-(9-2 z) z)-\omega^{2}(4-z)(5-z(z+3))\right)}{4\left(\omega-E^{\prime}\right)^{2}\left(\omega z-E^{\prime}\right)}, \\
& a_{\mathrm{R}}=\frac{-\omega^{6}(1-z)^{2}(8-z(16-(15-4 z) z))-E^{\prime} \omega^{5}\left(8-z\left(35-z\left(4 z^{3}-14 z^{2}+z+36\right)\right)\right)-4 E^{\prime 2} \omega^{4}}{4\left(E^{\prime}+\omega(1-z)\right)^{3}\left(\omega z-E^{\prime}\right)} \\
& +\frac{E^{\prime 4} \omega^{2}(6+(2-5 z) z)+3 E^{\prime 3} \omega^{3}(6-(2-z)(8-z) z)-E^{\prime 2} \omega^{4} z(24-z(66-(46-9 z) z))}{4\left(E^{\prime}+\omega(1-z)\right)^{3}\left(\omega z-E^{\prime}\right)} \\
& -\frac{E^{\prime 5} \omega(4-3 z)}{4\left(E^{\prime}+\omega(1-z)\right)^{3}\left(\omega z-E^{\prime}\right)}, \\
& a_{\mathrm{R}}^{\mathrm{L}}=m \omega \frac{E^{\prime}\left(\omega(2-(2-z) z)-E^{\prime} z\right)}{\left(E^{\prime}+\omega(1-z)\right)\left(\omega z-E^{\prime}\right)}, \\
& b_{\mathrm{L}}=-\frac{\left(\omega-E^{\prime}\right)\left(\left(E^{\prime}+\omega(1-z)\right)^{2}+\omega^{2}\right)}{\omega z-E^{\prime}} \text {, } \\
& b_{\mathrm{R}}=-\frac{\left(\omega-E^{\prime}\right)\left(\left(\omega-E^{\prime}\right)^{2}+\omega^{2}(1-z)^{2}\right)}{\omega z-E^{\prime}}, \\
& b_{\mathrm{R}}^{\mathrm{L}}=m \frac{2\left(\omega-E^{\prime}\right)\left(E^{\prime 2}+\left(\omega z-E^{\prime}\right)^{2}\right)}{E^{\prime}\left(\omega z-E^{\prime}\right)}, \\
& c_{\mathrm{L}}=\frac{\left(\omega-E^{\prime}\right)\left(\left(E^{\prime}+\omega(1-z)\right)^{2}+\omega^{2}\right)}{E^{\prime}+\omega(1-z)}, \\
& c_{\mathrm{R}}=\frac{\left(\omega-E^{\prime}\right)\left(\left(\omega-E^{\prime}\right)^{2}+\omega^{2}(1-z)^{2}\right)}{E^{\prime}+\omega(1-z)}+\frac{E^{\prime} \omega\left(\omega-E^{\prime}\right)\left(-2 \omega^{2}(1-z)-E^{\prime}\left(\omega z-E^{\prime}\right)\right)}{\left(E^{\prime}+\omega(1-z)\right)^{3}}, \\
& c_{\mathrm{R}}^{\mathrm{L}}=m \frac{2\left(\omega-E^{\prime}\right)\left(\omega z-2 E^{\prime}\right)}{E^{\prime}+\omega(1-z)}, \\
& d_{\mathrm{L}}=\left(\omega-E^{\prime}\right)\left(E^{\prime}+\omega(1-z)\right)-\frac{E^{\prime 3} \omega}{2\left(\omega-E^{\prime}\right)^{2}}+\frac{\omega^{3}\left(2 E^{\prime 2}(3-z)-4 E^{\prime} \omega-\omega^{2}(2-z(6-(4-z) z))\right)}{2\left(E^{\prime}-\omega\right)^{2}\left(E^{\prime}+\omega(1-z)\right)} \text {, } \\
& d_{\mathrm{R}}=\frac{\left(E^{\prime}-\omega\right)^{2}}{\left(E^{\prime}+\omega(1-z)\right)^{2}} d_{\mathrm{L}}-\frac{\omega\left(\omega z-E^{\prime}\right)^{2}\left(\left(\omega z-E^{\prime}\right)^{2}+2 \omega^{2}(1-z)\right)(2-z)}{2\left(E^{\prime}+\omega(1-z)\right)^{3}}, \\
& d_{\mathrm{R}}^{\mathrm{L}}=m \frac{\left(\omega z-2 E^{\prime}\right)^{2}}{E^{\prime}+\omega(1-z)} \text {. }
\end{aligned}
$$


The variable $z \leq 1$ is introduced to emphasize the forward direction of the relativistic electron,

$$
1-\cos \theta_{e} \equiv \frac{m}{E^{\prime}}(1-z)
$$

At $m \rightarrow 0$, the physical region of kinematical variables is given by

$$
m \leq E^{\prime} \leq \omega, \quad \frac{E^{\prime}}{\omega} \leq z \leq 1
$$

\section{Double-differential distribution in electromagnetic energy and electron angle}

To obtain the distribution with respect to the electromagnetic energy and electron angle, we use the neutrino energy $\omega^{\prime}$ instead of $k_{\gamma}$ in the triple-differential cross section, change the integration order, and integrate first over the electron energy. The final neutrino energy determines the total electromagnetic energy $E_{\mathrm{EM}}: E_{\mathrm{EM}}=E^{\prime}+k_{\gamma}=m+\omega-\omega^{\prime}$ and can be used to obtain $E_{\mathrm{EM}}$ distributions since $\mathrm{d} E_{\mathrm{EM}}=-\mathrm{d} \omega^{\prime}$.

In the limit of small electron mass, the neutrino energy and electron angle distribution is given by the following substitutions in Eqs. (58) and (59)':

$$
\tilde{\mathrm{I}}_{i} \underset{\omega \gg m}{\longrightarrow} \frac{\pi^{2}}{\omega^{3}}\left(a_{i}+\frac{b_{i}}{\left|\omega-\left(\omega-\omega^{\prime}\right)(2-\tilde{z})\right|}+c_{i} \ln \frac{1-\tilde{z}}{2-\tilde{z}}+\left(d_{i}+\frac{e_{i}}{\left|\omega-\left(\omega-\omega^{\prime}\right)(2-\tilde{z})\right|}\right) \ln \frac{\left|\frac{1-\tilde{z}}{2-\tilde{z}}-\frac{\omega^{\prime}}{\omega}\right|}{1-\frac{\omega^{\prime}}{\omega}}\right) \mathrm{d} \tilde{z} \mathrm{~d} \omega^{\prime},
$$

with the coefficients $a_{i}, b_{i}, c_{i}, d_{i}$, and $e_{i}$,

$$
\begin{aligned}
& a_{\mathrm{L}}=\frac{\left(2 \omega^{3}(1-\tilde{z})-\omega^{2} \omega^{\prime}(1-4 \tilde{z})-9 \omega \omega^{\prime 2}(5-2 \tilde{z})-\omega^{\prime 3}(23-18 \tilde{z})\right) \omega}{4 \omega^{\prime 2}}, \\
& a_{\mathrm{R}}=\frac{(1-\tilde{z})^{2}\left(-\omega(9-4 \tilde{z})+2 \omega^{\prime}(2-\tilde{z})\right) \omega}{4(2-\tilde{z})^{2}}, \\
& a_{\mathrm{R}}^{\mathrm{L}}=\frac{3-\tilde{z}}{2-\tilde{z}} m \omega, \\
& b_{\mathrm{L}}=\frac{1}{4} \omega\left(\omega-\omega^{\prime}\right)\left(-\omega(5-2 \tilde{z})+2 \omega^{\prime}(2-\tilde{z})\right) \text {, } \\
& b_{\mathrm{R}}=\frac{(1-\tilde{z})^{2}}{(2-\tilde{z})^{2}} b_{\mathrm{L}} \text {, } \\
& b_{\mathrm{R}}^{\mathrm{L}}=\frac{\omega(5-(5-2 \tilde{z}) \tilde{z})-2 \omega^{\prime}(5-(4-\tilde{z}) \tilde{z})}{2(2-\tilde{z})} m \omega, \\
& c_{\mathrm{L}}=-\frac{\omega\left(\omega^{3}(1-\tilde{z})+2 \omega^{2} \omega^{\prime} \tilde{z}+\omega \omega^{\prime 2}(31-(37-10 \tilde{z}) \tilde{z})+\omega^{\prime 3}(18-(26-9 \tilde{z}) \tilde{z})\right)}{2 \omega^{\prime 2}}-\frac{\omega^{2}\left(\omega-\omega^{\prime}\right)^{3}(1-\tilde{z})^{2}}{2 \omega^{\prime 3}} \text {, } \\
& c_{\mathrm{R}}=-\frac{\omega\left(\omega^{2}(1-\tilde{z})^{2}+\omega^{\prime 2}\right)}{2 \omega^{\prime}}, \\
& c_{\mathrm{R}}^{\mathrm{L}}=(2-\tilde{z}) \frac{\omega}{\omega^{\prime}} m \omega \\
& d_{\mathrm{L}}=\frac{\left(\omega-\omega^{\prime}\right)\left(\omega^{4}(1-\tilde{z})^{2}-\omega^{3} \omega^{\prime}(1-(3-2 \tilde{z}) \tilde{z})+\omega^{2} \omega^{\prime 2}(2-(1-\tilde{z}) \tilde{z})-\omega \omega^{\prime 3}(3-\tilde{z})+\omega^{\prime 4}(2-\tilde{z})\right)}{2 \omega^{\prime 3}}, \\
& d_{\mathrm{R}}=\frac{\left(\omega-\omega^{\prime}\right)\left(\left(\omega-\omega^{\prime}\right)^{2}(1-\tilde{z})^{2}+\omega^{\prime 2}\right)}{2 \omega^{\prime}}, \\
& d_{\mathrm{R}}^{\mathrm{L}}=\frac{m\left(\omega-\omega^{\prime}\right)\left(-\omega(2-\tilde{z})+\omega^{\prime}(3-\tilde{z})\right)}{\omega^{\prime}}, \\
& e_{\mathrm{L}}=-\frac{1}{2}\left(\omega-\omega^{\prime}\right)\left(\left(\omega-\omega^{\prime}\right)^{2}(2-\tilde{z})^{2}+\omega^{2}\right), \\
& e_{\mathrm{R}}=-\omega^{\prime} d_{\mathrm{R}} \text {, } \\
& e_{\mathrm{R}}^{\mathrm{L}}=m\left(\omega^{2}(2-(2-\tilde{z}) \tilde{z})-2 \omega \omega^{\prime}(3-(3-\tilde{z}) \tilde{z})+\omega^{\prime 2}(5-(4-\tilde{z}) \tilde{z})\right) \text {. }
\end{aligned}
$$


This approximation is valid only away from the elastic peak [cf. Eq. (70)] when

$$
|\tilde{z}-\tilde{Z}| \gg \frac{\omega^{\prime}}{\omega-\omega^{\prime}} \text {. }
$$

At $m \rightarrow 0$, the physical region is given by

$$
0 \leq \omega^{\prime} \leq \omega, \quad 1-\frac{\omega}{m} \leq \tilde{z} \leq 1 .
$$

We discuss the double-differential distribution in electromagnetic energy and electron angle keeping all electron mass terms in Appendix D.

\section{E. Double-differential distribution in photon energy and electron energy}

To obtain the distribution with respect to photon energy and electron energy, we can change the integration order and integrate the triple-differential cross section first over the electron scattering angle. In the limit of small electron mass, the leading terms of the photon energy and electron energy distribution are given by the following substitutions in Eqs. (58) and $(59)^{6}$ :

$$
\begin{aligned}
& \tilde{\mathrm{I}}_{\mathrm{L}} \underset{\omega \gg m}{\longrightarrow}\left(\frac{-29 E^{\prime 2}+8 E^{\prime} k_{\gamma}\left(\frac{\omega^{\prime}}{\omega}-3\right)+k_{\gamma}^{2}\left(\frac{\omega^{\prime 2}}{\omega^{2}}-6\right)}{12 E_{\mathrm{EM}}^{2}}+\frac{1}{2}\left(1+\frac{E^{\prime 2}}{E_{\mathrm{EM}}^{2}}\right) \ln \frac{2 E^{\prime} E_{\mathrm{EM}}}{m k_{\gamma}}\right) \mathcal{D}_{\gamma}, \\
& \tilde{\mathrm{I}}_{\mathrm{R}} \underset{\omega \gg m}{\longrightarrow}\left(\frac{-29 E^{\prime 2}+8 E^{\prime} k_{\gamma}\left(\frac{\omega}{\omega^{\prime}}-3\right)+k_{\gamma}^{2}\left(\frac{\omega^{2}}{\omega^{\prime 2}}-6\right)}{12 E_{\mathrm{EM}}^{2}}+\frac{1}{2}\left(1+\frac{E^{\prime 2}}{E_{\mathrm{EM}}^{2}}\right) \ln \frac{2 E^{\prime} E_{\mathrm{EM}}}{m k_{\gamma}}\right) \frac{\omega^{\prime 2}}{\omega^{2}} \mathcal{D}_{\gamma}, \\
& \tilde{\mathrm{I}}_{\mathrm{R}}^{\mathrm{L}} \underset{\omega \gg m}{\longrightarrow}\left(\frac{E^{\prime 2}\left(4 \frac{E_{\mathrm{EM}}^{2}}{\omega \omega^{\prime}}-1\right)-E^{\prime} k_{\gamma}\left(\frac{\omega}{\omega^{\prime}}-3\right)\left(\frac{\omega^{\prime}}{\omega}-3\right)+3 k_{\gamma}^{2}}{2 E_{\mathrm{EM}}^{2}}-\left(\frac{E^{\prime} E_{\mathrm{EM}}}{\omega \omega^{\prime}}+\frac{k_{\gamma}^{2}}{E_{\mathrm{EM}}^{2}}\right) \ln \frac{2 E^{\prime} E_{\mathrm{EM}}}{m k_{\gamma}}\right) \frac{m}{E_{\mathrm{EM}}} \frac{\omega^{\prime}}{\omega} \mathcal{D}_{\gamma},
\end{aligned}
$$

valid in the physical region, $0 \leq E^{\prime}+k_{\gamma} \leq \omega$, with the phase-space factor $\mathcal{D}_{\gamma}$,

$$
\mathcal{D}_{\gamma}=\pi^{2} \frac{\mathrm{d} k_{\gamma}}{k_{\gamma}} \frac{\mathrm{d} E^{\prime}}{\omega}
$$

We discuss the double-differential distribution in photon energy and electron energy keeping all electron mass terms in Appendix E.

\section{F. Double-differential distribution in photon energy and photon angle}

Besides the electron angle, the photon scattering angle $\theta_{\gamma}$ can be measured in principle. We consider the distribution with respect to the photon energy and the photon angle in the following. We present the double-differential distribution in photon energy and photon angle keeping all electron mass terms in Appendix F.

In the limit of small electron mass, the cross section is given by the following substitutions in Eqs. (58) and (59) ${ }^{6}$ :

$$
\tilde{\mathrm{I}}_{i} \underset{\omega \gg m}{\longrightarrow} \frac{\pi^{2}}{\omega^{3}}\left(a_{i}+b_{i} \ln \frac{m / 2}{\omega-k_{\gamma}(2-\bar{z})}\right) \frac{\mathrm{d} k_{\gamma}}{2 k_{\gamma}} \frac{\mathrm{d} \bar{z}}{(2-\bar{z})^{2}},
$$

with coefficients

$$
\begin{aligned}
a_{\mathrm{L}}= & -k_{\gamma}^{3}(1-\bar{z})(2-\bar{z})^{3}+k_{\gamma}^{2} \omega(2-3 \bar{z})(2-\bar{z})^{2}+4 k_{\gamma} \omega^{2} \bar{z}(2-\bar{z})-2 \omega^{3}(1+\bar{z}), \\
a_{\mathrm{R}}= & \frac{k_{\gamma}^{2}\left(-2 k_{\gamma}(1-\bar{z})(2-(2-\bar{z}) \bar{z})(2-\bar{z})-\omega(2-(2+(7-6 \bar{z}) \bar{z}) \bar{z})\right)}{6} \\
& +\frac{\omega^{2}\left(k_{\gamma}(2-\bar{z})(10-(24-(9+4 \bar{z}) \bar{z}) \bar{z})-\omega(12-(30-(15+2 \bar{z}) \bar{z}) \bar{z})\right)}{3(2-\bar{z})^{2}},
\end{aligned}
$$




$$
\begin{aligned}
a_{\mathrm{R}}^{\mathrm{L}} & =\frac{m\left(k_{\gamma}^{2}(3-2 \bar{z})(1-\bar{z})(2-\bar{z})^{2}-k_{\gamma} \omega(6-\bar{z}) \bar{z}(2-\bar{z})+\omega^{2}(6-\bar{z}) \bar{z}\right)}{2-\bar{z}}, \\
b_{\mathrm{L}} & =-\omega\left(k_{\gamma}^{2}(2-\bar{z})^{2}-2 k_{\gamma} \omega(2-\bar{z})+2 \omega^{2}\right), \\
b_{\mathrm{R}} & =\frac{(1-\bar{z})^{2}}{(2-\bar{z})^{2}} b_{\mathrm{L}}, \\
b_{\mathrm{R}}^{\mathrm{L}} & =\frac{2 m\left(k_{\gamma}^{2}(1-\bar{z})(2-\bar{z})^{3}-k_{\gamma} \omega(2-\bar{z})+\omega^{2}\right)}{2-\bar{z}},
\end{aligned}
$$

where the variable $\bar{z} \leq 1$ is introduced to emphasize the forward direction of the photon,

$$
1-\cos \theta_{\gamma} \equiv \frac{m}{\omega}(1-\bar{z}) .
$$

The photon angle with respect to the neutrino beam direction is bounded as

$$
\cos \theta_{\gamma} \geq 1-\frac{m}{k_{\gamma}}\left(1-\frac{k_{\gamma}}{\omega}\right),
$$

while the physical region for the photon energy is $0 \leq k_{\gamma} \leq \omega$.

\section{G. Photon energy spectrum}

Integrating the double-differential distribution in photon and electron energies over the electron energy, or the double-differential distribution in the photon energy and photon scattering angle over the angle, we obtain the photon energy spectrum. We present the photon energy spectrum keeping all electron mass terms in Appendix G. The leading terms in the electron mass expansion are given by the following substitutions in Eqs. (58) and (59):

$$
\begin{aligned}
& \tilde{\mathrm{I}}_{\mathrm{L}} \underset{\omega \gg m}{\longrightarrow} \frac{\pi^{2}}{\omega} g_{\mathrm{L}}\left(\frac{k_{\gamma}}{\omega}\right) \mathrm{d} k_{\gamma}, \\
& \tilde{\mathrm{I}}_{\mathrm{R}} \underset{\omega \gg m}{\longrightarrow} \frac{\pi^{2}}{\omega} g_{\mathrm{R}}\left(\frac{k_{\gamma}}{\omega}\right) \mathrm{d} k_{\gamma}, \\
& \tilde{\mathrm{I}}_{\mathrm{R}}^{\mathrm{L}} \underset{\omega \gg m}{\longrightarrow} \frac{\pi^{2}}{\omega} \frac{m}{\omega} g_{\mathrm{R}}^{\mathrm{L}}\left(\frac{k_{\gamma}}{\omega}\right) \mathrm{d} k_{\gamma},
\end{aligned}
$$

with functions $g_{\mathrm{L}}(x), g_{\mathrm{R}}(x)$, and $g_{\mathrm{R}}^{\mathrm{L}}(x)$ derived first in the present paper ${ }^{6}$,

$$
\begin{aligned}
g_{\mathrm{L}}(x)= & \frac{(1-x)\left(x^{2}-20 x-53\right)}{12 x}-\left(3+\frac{1}{x}\right) \ln x-\frac{x^{2}+x-2}{2 x} \ln \frac{2 \omega(1-x)}{m}+\ln \frac{2 \omega}{m} \ln x+\frac{\pi^{2}}{6}-\mathrm{Li}_{2} x, \\
g_{\mathrm{R}}(x)= & -\frac{(1-x)\left(37 x^{2}+223 x+73\right)}{36 x}-\left(\frac{1}{3 x}+\frac{9+5 x}{2}\right) \ln x+\frac{(1-x)\left(x^{2}+4 x+1\right)}{3 x} \ln \frac{2 \omega(1-x)}{m} \\
& +\left(\ln \frac{2 \omega}{m} \ln x+\frac{\pi^{2}}{6}-\mathrm{Li}_{2} x\right)(1+x), \\
g_{\mathrm{R}}^{\mathrm{L}}(x)= & \frac{(1-x)(11-13 x)}{4 x}+\frac{1-2 x}{2 x} \ln x-\frac{(1-x)^{2}}{x} \ln \frac{2 \omega(1-x)}{m} .
\end{aligned}
$$

The integral of the photon energy spectrum obtained from Eqs. (88)-(90) is infrared divergent if extended to arbitrary small photon energy. The total NLO cross section is obtained by implementing an infrared regulator and including the (separately infrared divergent) virtual correction from Sec. III.

\section{H. Electron energy spectrum}

All of our following calculations for neutrino and antineutrino scattering contain the same IR contribution arising from the soft-photon phase space, when the elastic process (without radiation) and scattering with bremsstrahlung are experimentally indistinguishable. The soft-photon contribution has to be accounted for in differential cross sections with respect to one kinematical variable (except for the photon energy spectrum of Sec. IV G, where one simply evaluates the spectrum above a chosen minimum photon energy). The amplitude $\mathrm{T}_{\text {soft }}^{1 \gamma}$ for the radiation of one soft photon with energy $k_{\gamma} \leq \varepsilon$, where $\varepsilon \ll m, \omega$ denotes a cutoff regulator, can be expressed in factorizable form as 


$$
\mathrm{T}_{\mathrm{soft}}^{1 \gamma}=\left[\frac{\left(\varepsilon^{*} \cdot p^{\prime}\right)}{\left(k_{\gamma} \cdot p^{\prime}\right)}-\frac{\left(\varepsilon^{*} \cdot p\right)}{\left(k_{\gamma} \cdot p\right)}\right] e \mathrm{~T}
$$

where $\mathrm{T}$ corresponds to the amplitude without radiation. The corresponding contribution $\mathrm{d} \sigma_{\text {soft }}^{\nu_{t} e \rightarrow \nu_{t} e \gamma}$ to the bremsstrahlung spectrum is given by

$$
\mathrm{d} \sigma_{\mathrm{soft}}^{\nu_{\ell} e \rightarrow \nu_{\ell} e \gamma}=\frac{\alpha}{\pi} \delta_{s} \mathrm{~d} \sigma_{\mathrm{LO}}^{\nu_{\ell} e \rightarrow \nu_{\ell} e},
$$

with the soft correction factor $\delta_{s}[21,25,29,37]$,

$$
\begin{aligned}
\delta_{s}= & \frac{1}{\beta}\left(\operatorname{Li}_{2} \frac{1-\beta}{1+\beta}-\frac{\pi^{2}}{6}\right)-\frac{2}{\beta}\left(\beta-\frac{1}{2} \ln \frac{1+\beta}{1-\beta}\right) \ln \frac{2 \varepsilon}{\lambda} \\
& +\frac{1}{2 \beta} \ln \frac{1+\beta}{1-\beta}\left(1+\ln \frac{\rho(1+\beta)}{4 \beta^{2}}\right)+1 .
\end{aligned}
$$

The velocity $\beta$ of Eq. (34) (and $\rho=\sqrt{1-\beta^{2}}$ ),

$$
\beta=\sqrt{1-\frac{m^{2}}{\bar{E}^{2}}}
$$

now describes either electron or electromagnetic energy spectra and $\bar{E}$ stands for the corresponding energy, i.e., $\bar{E}=E^{\prime}$ or $\bar{E}=E_{\mathrm{EM}}$. Note the exact cancellation of the IR divergence in the sum of the vertex correction and the softphoton emission; i.e., $\delta_{s}+\delta_{v}$ does not depend on the fictitious photon mass $\lambda$ [114-117]. The correction of Eq. (96) comes entirely from the first (factorizable) terms in Eqs. (61)-(63) and still contains an unphysical dependence on the photon energy cutoff $\varepsilon$.

For further evaluation of the electron angle distributions, we introduce the four-vector $l$ [22],

$$
l=k+p-p^{\prime}=\left(l_{0}, \vec{f}\right)
$$

with the laboratory frame values,

$$
\begin{gathered}
l_{0}=m+\omega-E^{\prime} \\
f^{2}=|\vec{f}|^{2}=\omega^{2}+\beta^{2} E^{\prime 2}-2 \omega \beta E^{\prime} \cos \theta_{e} .
\end{gathered}
$$

Besides the soft-photon correction, the first factorizable terms in Eqs. (61)-(63) contribute from the region $k_{\gamma} \geq \varepsilon$. It is convenient to split this contribution into two parts. There are no restrictions on the phase-space integration in region I: $l^{2}=l_{0}^{2}-f^{2} \geq 2 \varepsilon\left(l_{0}+f\right)$. In region II: $l^{2} \leq 2 \varepsilon\left(l_{0}+f\right)$, which includes the region of scattering with elastic kinematics, the phase space of the final photon is bounded by

$$
\cos \gamma \geq \frac{1}{f}\left(l_{0}-\frac{l^{2}}{2 \varepsilon}\right)
$$

where $\gamma$ is the angle between $\vec{f}$ and $\vec{k}_{\gamma}$. The bremsstrahlung contribution from region I, $\mathrm{d} \sigma_{\mathrm{I}}^{\nu_{\ell} e \rightarrow \nu_{\ell} e \gamma}$, cancels the $\ln \varepsilon$ divergence of the soft-photon correction. It may be written as the sum of factorizable and nonfactorizable corrections,

$$
\mathrm{d} \sigma_{\mathrm{I}}^{\nu_{\ell} e \rightarrow \nu_{\ell} e \gamma}=\frac{\alpha}{\pi} \delta_{\mathrm{I}} \mathrm{d} \sigma_{\mathrm{LO}}^{\nu_{\ell} e \rightarrow \nu_{\ell} e}+\mathrm{d} \sigma_{\mathrm{I}, \mathrm{NF}}^{\nu_{\ell} e \rightarrow \nu_{e} e \gamma}
$$

The factorizable correction $\delta_{\mathrm{I}}$ is obtained from the first, factorizable, terms in Eqs. (61)-(63), evaluating kinematical factors $I_{L}, I_{R}, I_{R}^{L}$ in the kinematics of the elastic $2 \rightarrow 2$ process,

$$
\delta_{\mathrm{I}}=\frac{2}{\beta}\left(\beta-\frac{1}{2} \ln \frac{1+\beta}{1-\beta}\right) \ln \frac{2(1+\beta) \varepsilon}{\beta m\left(1+\cos \delta_{0}\right)},
$$

where the angle $\delta_{0}$ is given by

$$
\cos \delta_{0}=\frac{\omega^{2}-\beta^{2} E^{\prime 2}-l_{0}^{2}}{2 \beta E^{\prime} l_{0}} .
$$

The nonfactorizable part $\mathrm{d} \sigma_{\mathrm{I}, \mathrm{NF}}^{\nu_{\ell} e \rightarrow \nu_{\ell} e \gamma}$ is discussed below. The bremsstrahlung contribution from region II can be expressed in factorizable form

$$
\mathrm{d} \sigma_{\mathrm{II}}^{\nu_{\ell} e \rightarrow \nu_{\ell} e \gamma}=\frac{\alpha}{\pi} \delta_{\mathrm{II}} \mathrm{d} \sigma_{\mathrm{LO}}^{\nu_{\ell} e \rightarrow \nu_{\ell} e},
$$

where

$$
\begin{aligned}
\delta_{\mathrm{II}}= & \frac{1}{\beta}\left(\left(\frac{1}{2}+\ln \frac{\rho\left(1+\cos \delta_{0}\right)}{4 \beta}\right) \ln \frac{1-\beta}{1+\beta}-\mathrm{Li}_{2} \frac{1-\beta}{1+\beta}\right. \\
& \left.-\mathrm{Li}_{2} \frac{\cos \delta_{0}-1}{\cos \delta_{0}+1}+\mathrm{Li}_{2}\left(\frac{\cos \delta_{0}-1}{\cos \delta_{0}+1} \frac{1+\beta}{1-\beta}\right)+\frac{\pi^{2}}{6}\right) \\
& +\ln \frac{1-\beta \cos \delta_{0}}{\rho}-1 .
\end{aligned}
$$

Consequently, the complete electron energy spectrum is given by

$$
\begin{aligned}
& \mathrm{d} \sigma_{\mathrm{LO}}^{\nu_{\ell} e \rightarrow \nu_{\ell} e \gamma}+\mathrm{d} \sigma_{\mathrm{NLO}}^{\nu_{\ell} e \rightarrow \nu_{\ell} e} \\
& =\left[1+\frac{\alpha}{\pi}\left(\delta_{v}+\delta_{s}+\delta_{\mathrm{I}}+\delta_{\mathrm{II}}\right)\right] \mathrm{d} \sigma_{\mathrm{LO}}^{\nu_{\ell} e \rightarrow \nu_{\ell} e} \\
& \quad+\mathrm{d} \sigma_{v}^{\nu_{\ell} e \rightarrow \nu_{\ell} e}+\mathrm{d} \sigma_{\mathrm{dyn}}^{\nu_{\ell} e \rightarrow \nu_{\ell} e}+\mathrm{d} \sigma_{\mathrm{NF}}^{\nu_{\ell} e \rightarrow \nu_{\ell} e \gamma}
\end{aligned}
$$

and does not depend on the unphysical parameters $\varepsilon$ and $\lambda$. We remark that although individual corrections contain double logarithms, i.e.,

$\delta_{v} \underset{\beta \rightarrow 1}{\sim}-\frac{1}{8} \ln ^{2}(1-\beta), \quad \underset{\delta_{\beta \rightarrow 1}^{\sim}}{\sim}-\frac{1}{4} \ln ^{2}(1-\beta)$,

$\delta_{\text {II }} \underset{\beta \rightarrow 1}{\sim} \frac{3}{8} \ln ^{2}(1-\beta)$,

the complete cross-section correction is free from such Sudakov double logarithms [118,119]. In Appendix H, 
we obtain the remaining nonfactorizable piece $\mathrm{d} \sigma_{\mathrm{NF}}^{\nu_{\ell} e \rightarrow \nu_{\ell} e \gamma}$ from the region of hard photons $\left(k_{\gamma} \geq \varepsilon\right)$, which contains $\mathrm{d} \sigma_{\mathrm{I}, \mathrm{NF}}^{\nu_{\ell} \rightarrow \nu_{e} e \gamma}$ as well as the contribution beyond the first factorizable terms in Eqs. (61)-(63), integrating the electron angle and electron energy distribution over the variable $f$ (equivalent to the electron scattering angle $\theta_{e}$ ), and retaining all electron mass terms.

The resulting correction to the electron energy spectrum reproduces the result of Ref. [25] in the limit $m \rightarrow 0$, $E^{\prime} / \omega=$ const. Besides the closed fermion loop contribution of Secs. III B and IIIC, it is represented by the following substitutions in Eqs. (58) and (59):

$$
\begin{aligned}
& \tilde{\mathrm{I}}_{\mathrm{L}} \underset{\omega \gg m}{\longrightarrow} \frac{\pi^{2}}{\omega} f_{-}\left(\frac{E^{\prime}}{\omega}\right) \mathrm{d} E^{\prime}, \\
& \tilde{\mathrm{I}}_{\mathrm{R}} \underset{\omega \gg m}{\longrightarrow} \frac{\pi^{2}}{\omega}\left(1-\frac{E^{\prime}}{\omega}\right)^{2} f_{+}\left(\frac{E^{\prime}}{\omega}\right) \mathrm{d} E^{\prime}, \\
& \tilde{\mathrm{I}}_{\mathrm{R}}^{\mathrm{L}} \underset{\omega \gg m}{\longrightarrow}-\frac{\pi^{2}}{\omega} \frac{m}{\omega} \frac{E^{\prime}}{\omega} f_{+}^{-}\left(\frac{E^{\prime}}{\omega}\right) \mathrm{d} E^{\prime},
\end{aligned}
$$

with functions $f_{-}(x), f_{+}(x)$ [25], and $f_{+}^{-}(x)$ derived first in the present paper,

$$
\begin{aligned}
f_{-}(x)= & -\frac{2}{3} \ln \frac{2 \omega}{m}+\left(\ln \frac{1-x}{\sqrt{x}}+\frac{x}{2}+\frac{1}{4}\right) \ln \frac{2 \omega}{m}-\frac{1}{2}\left(\mathrm{Li}_{2}(x)-\frac{\pi^{2}}{6}\right)+\frac{x^{2}}{24}-\frac{11 x}{12}-\frac{47}{36} \\
& -\frac{1}{2} \ln ^{2} \frac{1-x}{x}-\left(\frac{x}{2}+\frac{23}{12}\right) \ln (1-x)+x \ln x, \\
(1-x)^{2} f_{+}(x)= & -\frac{2}{3}(1-x)^{2} \ln \frac{2 \omega}{m}+\left(\frac{x-1}{2}+(1-x)^{2} \ln (1-x)\right) \ln \frac{2 \omega}{m}-\frac{(1-x)^{2}}{2} \ln \frac{1-x}{x^{2}} \ln (1-x) \\
& +\left((1-x) x-\frac{1}{2}\right)\left(\operatorname{Li}_{2}(x)+\ln \frac{2 \omega x}{m} \ln x-\frac{\pi^{2}}{6}\right)+\left(x^{2}+\frac{x}{2}-\frac{3}{4}\right) \ln x \\
& -\frac{31-49 x}{72}(1-x)+\frac{1-x}{3}\left(5 x-\frac{7}{2}\right) \ln (1-x), \\
-x f_{+}^{-}(x)= & 2+2 \ln x+\left(x-\ln x-\frac{1}{2}\right) \ln \frac{2 \omega x}{m}+\left(\frac{3}{2} x+\frac{1}{2}-x \ln \frac{2 \omega x}{m}\right) \ln \frac{1-x}{x}+\frac{1}{2} x \ln ^{2}(1-x) \\
& +(x-1)\left(\operatorname{Li}_{2}(x)-\frac{\pi^{2}}{6}+\frac{5}{4}\right) .
\end{aligned}
$$

We observe that in exactly forward kinematics at electron threshold, when $E^{\prime}=m$, the energy spectrum is given by the nonfactorizable contribution from the electromagnetic vertex and closed fermion loops,

$$
\begin{aligned}
& \mathrm{d} \sigma_{\mathrm{LO}}^{\nu_{\ell} e \rightarrow \nu_{\ell} e \gamma}+\mathrm{d} \sigma_{\mathrm{NLO}}^{\nu_{\ell} e \rightarrow \nu_{\ell} e} \underset{E^{\prime} \rightarrow m}{\longrightarrow} \mathrm{d} \sigma_{\mathrm{NLO}}^{\nu_{\ell} e \rightarrow \nu_{\ell} e} \rightarrow \mathrm{d} \sigma_{\mathrm{LO}}^{\nu_{\ell} e \rightarrow \nu_{\ell} e} \\
& \quad+\mathrm{d} \sigma_{v}^{\nu_{\ell} e \rightarrow \nu_{\ell} e}+\mathrm{d} \sigma_{\mathrm{dyn}}^{\nu_{\ell} e \rightarrow \nu_{\ell} e}
\end{aligned}
$$

with $f_{2}(0)=1 / 2$ in Eqs. (35), (37), (38) and $\Pi\left(0, m_{f}\right)$, $\hat{\Pi}_{\gamma \gamma}^{(3)}(0), \hat{\Pi}_{3 \gamma}^{(3)}(0)$ of Eqs. (42), (47). This equation provides a universal limit for electron energy and electromagnetic energy spectra.

The electron energy spectrum has the following logarithmically divergent behavior near its end point $E^{\prime} \leq E_{0}^{\prime}=m+\frac{2 \omega^{2}}{m+2 \omega}$ :

$\frac{\mathrm{d} \sigma_{\mathrm{LO}}^{\nu_{\ell} e \rightarrow \nu_{\ell} e \gamma}+\mathrm{d} \sigma_{\mathrm{NLO}}^{\nu_{\ell} e \rightarrow \nu_{\ell} e}}{\mathrm{~d} \sigma_{\mathrm{LO}}^{\nu_{\ell} e \rightarrow \nu_{\ell} e}} \approx-\frac{\alpha}{\pi} \frac{2}{\beta}\left(\beta-\frac{1}{2} \ln \frac{1+\beta}{1-\beta}\right) \ln \frac{E_{0}^{\prime}-E^{\prime}}{m}$,

as determined by infrared logarithms in Eqs. (36) and (96).

\section{Electromagnetic energy spectrum}

We evaluate the bremsstrahlung cross section with respect to the sum of electron and photon energies considering the final neutrino energy spectrum instead of the electron energy spectrum [22]; see Sec. IV D for explanations. For the neutrino scattering angle distributions, we introduce the four-vector $\tilde{l}$,

$$
\tilde{l}=k+p-k^{\prime}=\left(\tilde{l}_{0}, \overrightarrow{\tilde{f}}\right)
$$

with the laboratory frame values,

$$
\begin{gathered}
\tilde{l}_{0}=E_{\mathrm{EM}} \\
\tilde{f}^{2}=|\overrightarrow{\tilde{f}}|^{2}=\omega^{2}+\omega^{\prime 2}-2 \omega \omega^{\prime} \cos \theta_{\nu}
\end{gathered}
$$

Note the difference between the neutrino scattering angle in the elastic process $\left[\Theta_{\nu}\right.$ of Eq. (3) $]$ and in the scattering with radiation $\left(\theta_{\nu}\right)$.

Below the end point of maximal electron energy, $E_{\mathrm{EM}} \leq E_{0}^{\prime}=m+\frac{2 \omega^{2}}{m+2 \omega}$, we can use the same integration 
technique as in Ref. [22]. Above the end point, the photon energy is bounded from below $k_{\gamma} \geq E_{\mathrm{EM}}-E_{0}^{\prime}$, and there is no corresponding elastic process as well as no contribution from the soft region. We consider these two regions separately in the following.

\section{Below electron end point: $E_{\mathrm{EM}} \leq E_{0}^{\prime}=m+\frac{2 \omega^{2}}{m+2 \omega}$}

The contribution from the soft-photon region $k_{\gamma} \leq \varepsilon$ is given by Eqs. (95) and (96). We split the integration region with $k_{\gamma} \geq \varepsilon$ for factorizable terms in Eqs. (61)-(63) into two regions similar to Sec. IV H. In region I: $\tilde{l}^{2}-m^{2}=$ $\tilde{l}_{0}^{2}-\tilde{f}^{2}-m^{2} \geq 2 \varepsilon\left(\tilde{l}_{0}+\tilde{f}\right)$, there are no restrictions on the phase space. In region II: $\tilde{l}^{2}-m^{2} \leq 2 \varepsilon\left(\tilde{l}_{0}+\tilde{f}\right)$, the phase space of the final neutrino is restricted to

$$
\cos \tilde{\gamma} \geq \frac{1}{\tilde{f}}\left(\tilde{l}_{0}-\frac{\tilde{l}^{2}-m^{2}}{2 \varepsilon}\right),
$$

where $\tilde{\gamma}$ is the angle between $\overrightarrow{\tilde{f}}$ and $\vec{k}_{\gamma}$. The correction factor from region II, $\delta_{\mathrm{II}}$ [cf. Eq. (105)], is given by

$$
\delta_{\mathrm{II}}=-\frac{1}{\beta}\left(\beta-\frac{1}{2} \ln \frac{1+\beta}{1-\beta}\right) \ln \frac{1+\beta}{1-\beta} .
$$

Here $\beta$ is expressed in terms of electromagnetic energy as in Eq. (97). As for the electron energy spectrum, the bremsstrahlung contribution from region I may be written as the sum of factorizable and nonfactorizable corrections; cf. Eq. (102). The factorizable correction $\delta_{\mathrm{I}}$ is obtained from the first factorizable terms in Eqs. (61)-(63), evaluating kinematical factors $\mathrm{I}_{\mathrm{L}}, \mathrm{I}_{\mathrm{R}}, \mathrm{I}_{\mathrm{R}}^{\mathrm{L}}$ in the kinematics of the elastic $2 \rightarrow 2$ process,

$$
\delta_{\mathrm{I}}=\frac{2}{\beta}\left(\beta-\frac{1}{2} \ln \frac{1+\beta}{1-\beta}\right) \ln \frac{\varepsilon}{m} .
$$

In Appendix I we evaluate the remaining nonfactorizable piece $\mathrm{d} \sigma_{\mathrm{NF}}^{\nu_{\ell} e \rightarrow \nu_{e} e \gamma}$ of the electromagnetic energy spectrum below the electron end point, performing straightforward integrations and keeping all electron mass terms. It accounts for the region of hard photons $\left(k_{\gamma} \geq \varepsilon\right)$ and contains $\mathrm{d} \sigma_{\mathrm{I}, \mathrm{NF}}^{\nu_{\ell} e \rightarrow \nu_{\ell} e \gamma}$ as well as the contribution beyond the first factorizable terms in Eqs. (61)-(63).

The resulting correction to the electromagnetic energy spectrum reproduces the result of Refs. [27,28] in the limit $m \rightarrow 0, E_{\mathrm{EM}} / \omega=$ const. Besides the closed fermion loop contribution of Secs. III B and III C, it is represented by the following substitutions in Eqs. (58) and (59):

$$
\begin{aligned}
& \tilde{\mathrm{I}}_{\mathrm{L}} \underset{\omega \gg m}{\longrightarrow} \frac{\pi^{2}}{\omega} f_{\mathrm{L}}\left(\frac{E_{\mathrm{EM}}}{\omega}\right) \mathrm{d} E_{\mathrm{EM}}, \\
& \tilde{\mathrm{I}}_{\mathrm{R}} \underset{\omega \gg m}{\longrightarrow} \frac{\pi^{2}}{\omega}\left(1-\frac{E_{\mathrm{EM}}}{\omega}\right)^{2} f_{\mathrm{R}}\left(\frac{E_{\mathrm{EM}}}{\omega}\right) \mathrm{d} E_{\mathrm{EM}},
\end{aligned}
$$

$$
\tilde{\mathrm{I}}_{\mathrm{R}}^{\mathrm{L}} \underset{\omega \gg m}{\longrightarrow}-\frac{\pi^{2}}{\omega} \frac{m}{\omega} \frac{E_{\mathrm{EM}}}{\omega} f_{\mathrm{R}}^{\mathrm{L}}\left(\frac{E_{\mathrm{EM}}}{\omega}\right) \mathrm{d} E_{\mathrm{EM}},
$$

with functions $f_{\mathrm{L}}(x), f_{\mathrm{R}}(x)$ [27,28], and $f_{\mathrm{R}}^{\mathrm{L}}(x)$ derived first in the present work ${ }^{6}$,

$$
\begin{aligned}
& f_{\mathrm{L}}(x)=\frac{3 x^{2}-30 x+23}{72}-\frac{2}{3} \ln \frac{2 \omega x}{m}-\frac{\pi^{2}}{6}, \\
& f_{\mathrm{R}}(x)=\frac{-4 x^{2}-16 x+23}{72(1-x)^{2}}-\frac{2}{3} \ln \frac{2 \omega x}{m}-\frac{\pi^{2}}{6}, \\
& f_{\mathrm{R}}^{\mathrm{L}}(x)=\frac{x^{2}+3 x-3}{4 x^{2}}-\frac{3}{2} \ln \frac{2 \omega x}{m}-\frac{\pi^{2}}{6} .
\end{aligned}
$$

In exactly forward kinematics at electromagnetic energy threshold when $E_{\mathrm{EM}}=m$, the electromagnetic energy spectrum coincides with the electron energy spectrum; see Eq. (115).

Just below electron end point $\left(E_{\mathrm{EM}}<E_{0}^{\prime}=m+\right.$ $\left.\frac{2 \omega^{2}}{m+2 \omega} \approx \omega\right)$, the electromagnetic energy spectrum, besides the closed fermion loop contribution, is given by the following substitutions in the nonfactorizable correction ${ }^{6}$ :

$$
\begin{aligned}
& \tilde{\mathrm{I}}_{\mathrm{L}} \underset{\omega \gg m}{\longrightarrow}-\frac{\pi^{2}}{3}\left(\ln \frac{4 \omega^{2}}{m^{2}}+\frac{\pi^{2}}{2}+\frac{1}{6}\right) \frac{\mathrm{d} E_{\mathrm{EM}}}{\omega}, \\
& \tilde{\mathrm{I}}_{\mathrm{R}} \underset{\omega \gg m}{\longrightarrow} \frac{\pi^{2}}{24} \frac{\mathrm{d} E_{\mathrm{EM}}}{\omega}, \\
& \tilde{\mathrm{I}}_{\mathrm{R}}^{\mathrm{L}} \underset{\omega \gg m}{\longrightarrow} \frac{\pi^{2}}{4} \frac{m}{\omega}\left(3 \ln \frac{4 \omega^{2}}{m^{2}}+\frac{2 \pi^{2}}{3}-1\right) \frac{\mathrm{d} E_{\mathrm{EM}}}{\omega} .
\end{aligned}
$$

Equations (129) and (130) are in agreement with the similar limit taken from the result of Refs. [27,28].

\section{Above electron end point: $E_{\mathrm{EM}}>E_{0}^{\prime}=m+\frac{2 \omega^{2}}{m+2 \omega}$}

Above the electron end point energy, the corresponding elastic process is kinematically forbidden. For $\omega \gg m$, this region is relatively small but finite,

$$
E_{\mathrm{EM}}-E_{0}^{\prime} \leq \frac{1}{1+\frac{m}{2 \omega}} \frac{m}{2}<\frac{m}{2}
$$

Since the photon energy is bounded from below in this region, $k_{\gamma}>E_{\mathrm{EM}}-E_{0}^{\prime}$, the calculation does not require IR regularization. We present the electromagnetic energy spectrum above the electron end point keeping all electron mass terms in Appendix J.

The electromagnetic energy spectrum has the following logarithmically divergent behavior just above the electron end point $E_{\mathrm{EM}}>E_{0}^{\prime}=m+\frac{2 \omega^{2}}{m+2 \omega}$ :

$$
\frac{\mathrm{d} \sigma_{\mathrm{LO}}^{\nu_{\ell} e \rightarrow \nu_{\ell} e \gamma}}{\mathrm{d} \sigma_{\mathrm{LO}}^{\nu_{\ell} e \rightarrow \nu_{\ell} e}} \approx \frac{\alpha 2}{\pi} \frac{2}{\beta}\left(\beta-\frac{1}{2} \ln \frac{1+\beta}{1-\beta}\right) \ln \frac{E_{\mathrm{EM}}-E_{0}^{\prime}}{m} .
$$




\section{J. Absolute cross section}

The resulting total cross-section correction, besides closed fermion loop contributions, in the ultrarelativistic limit is given by the following substitutions in Eqs. (58) and (59) for $\tilde{I}_{L}, \tilde{I}_{R}[25]$, and $\tilde{I}_{R}^{L}$ derived first in the present paper ${ }^{6}$ :

$$
\begin{aligned}
& \tilde{\mathrm{I}}_{\mathrm{L}} \underset{\omega \gg m}{\longrightarrow} \frac{\pi^{2}}{24}\left(19-4 \pi^{2}-16 \ln \frac{2 \omega}{m}\right), \\
& \tilde{\mathrm{I}}_{\mathrm{R}} \underset{\omega \gg m}{\longrightarrow} \frac{\pi^{2}}{72}\left(19-4 \pi^{2}-16 \ln \frac{2 \omega}{m}\right)+\frac{\pi^{2}}{3}, \\
& \tilde{\mathrm{I}}_{\mathrm{R}}^{\mathrm{L}} \underset{\omega \gg m}{\longrightarrow}-\frac{\pi^{2}}{24} \frac{m}{\omega}\left(15-2 \pi^{2}-36 \ln \frac{2 \omega}{m}\right) .
\end{aligned}
$$

Factors $\tilde{\mathrm{I}}_{\mathrm{L}}$ and $\tilde{\mathrm{I}}_{\mathrm{R}}$ of Eqs. (134) and (135) can be obtained integrating Eqs. (126) and (127) or Eqs. (112) and (113) over the energy variable. To evaluate the factor $\tilde{I}_{R}^{L}$, one has to regulate the logarithmic mass singularity properly or take the limit from the general expression of Appendix K. Note the absence of double logarithms in the resulting crosssection correction in Eqs. (126)-(128) and (134)-(136), although individual corrections contain them; cf. Eq. (108). Note also that the total elastic cross section at leading order is given by the following substitutions in Eqs. (14) and (15):
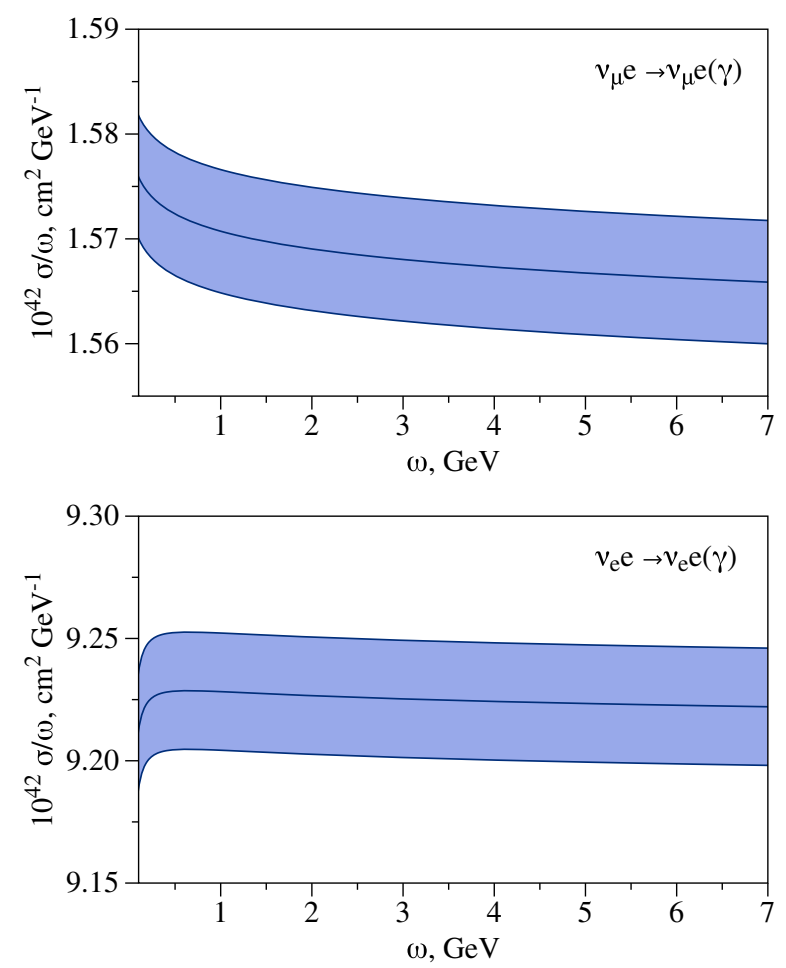

$\int \mathrm{d} \omega^{\prime} \mathrm{I}_{\mathrm{L}} \underset{\omega \gg m}{\longrightarrow} \omega, \quad \int \mathrm{d} \omega^{\prime} \mathrm{I}_{\mathrm{R}} \underset{\omega \gg m}{\longrightarrow} \frac{\omega}{3}, \quad \int \mathrm{d} \omega^{\prime} \mathrm{I}_{\mathrm{R}}^{\mathrm{L}} \underset{\omega \gg m}{\longrightarrow}-\frac{m}{2}$.

Results for the absolute cross section including the electron mass dependence are presented in Appendix K.

\section{ILLUSTRATIVE RESULTS}

Our results may be used to compute absolute and differential cross sections for neutrino-electron scattering over a broad range of energies and experimental setups. We focus on the application to flux normalization at accelerator-based neutrino experiments in Secs. VA through V $\mathrm{C}$ and discuss radiative corrections in the context of new physics searches in Sec. VD.

\section{A. Total cross section: Energy dependence and error analysis}

The total cross sections for $\nu_{\mu} e, \nu_{e} e, \bar{\nu}_{\mu} e$, and $\bar{\nu}_{e} e$ scattering are shown in Fig. 8. For $\omega \gg m$, cross sections grow approximately linearly with neutrino beam energy. As a benchmark point, we determine at $\omega=1 \mathrm{GeV}$

$$
\begin{aligned}
& \sigma^{\nu_{\mu} e \rightarrow \nu_{\mu} e(\gamma)}(\omega=1 \mathrm{GeV}) \\
& =\left[1.5707 \times 10^{-42} \mathrm{~cm}^{2}\right] \\
& \quad \times\left[1 \pm 0.0037_{\mathrm{had}} \pm 0.0007_{\mathrm{EW}} \pm 0.00007_{\mathrm{pert}}\right] .
\end{aligned}
$$
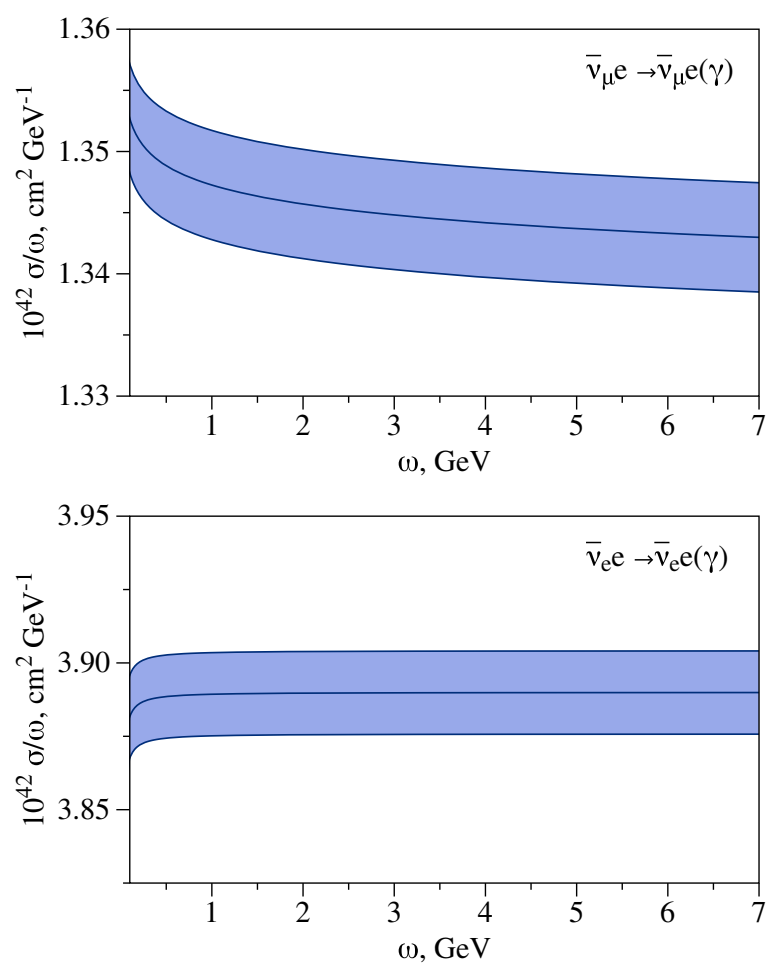

FIG. 8. Total cross section in the (anti)neutrino-electron scattering processes $\nu_{\mu} e \rightarrow \nu_{\mu} e\left(X_{\gamma}\right), \nu_{e} e \rightarrow \nu_{e} e\left(X_{\gamma}\right), \bar{\nu}_{\mu} e \rightarrow \bar{\nu}_{\mu} e\left(X_{\gamma}\right)$, and $\bar{\nu}_{e} e \rightarrow \bar{\nu}_{e} e\left(X_{\gamma}\right)$ as a function of (anti)neutrino beam energy $\omega$. 
The cross section is evaluated using four-flavor QCD, with running QED and QCD couplings $\alpha(\mu)$ and $\alpha_{s}(\mu)$ evaluated using two and five loop running, respectively, with $\alpha(2 \mathrm{GeV})=1 / 133.309$ and $\alpha_{s}(2 \mathrm{GeV})=0.3068$. The uncertainties in Eq. (138) are from the following: (i) the hadronic parameter $\hat{\Pi}_{3 \gamma}^{(3)}(0) / \hat{\Pi}_{\gamma \gamma}^{(3)}(0)$ in Eq. (54) and from $\hat{\Pi}_{\gamma \gamma}^{(3)}(0)$ in Eq. $(53)^{7}$; (ii) from uncertainties in the four-fermion operator coefficients $c_{\mathrm{L}}^{\nu_{\ell} \ell^{\prime}}, c_{\mathrm{R}}$ in Table I; and (iii) from higher-order perturbative corrections, estimated by varying renormalization scale $\mu_{0}^{2} / 2<\mu^{2}<2 \mu_{0}^{2}$, where $\mu_{0}=2 \mathrm{GeV}$. For simplicity, we evaluate the light-quark contribution of Eq. (47) neglecting NLO electroweak corrections and renormalization group corrections to the four-fermion operator coefficients, taking for definiteness $\mathrm{G}_{\mathrm{F}}=1.1663787 \times 10^{-5} \mathrm{GeV}^{-2}$ and $\sin ^{2} \theta_{W}=0.23112$ in Eqs. (47) and (48); it is straightforward to include these corrections, whose impact is given by the few permille shift in the coefficients [47], times the $\sim 1 \%$ fractional contribution of light quarks to the cross section. The charm-quark contribution in Eq. (42) is evaluated including the $\mathcal{O}\left(\alpha_{s}\right)$ and $\mathcal{O}\left(\alpha_{s}^{2}\right)$ corrections from Appendix A and using the $\overline{\mathrm{MS}}$ mass $\hat{m}_{c}(2 \mathrm{GeV})=$ $1.096 \mathrm{GeV}$ [corresponding to $\hat{m}_{c}\left(\hat{m}_{c}\right)=1.28(2) \mathrm{GeV}$ [120] ]. The fractional uncertainty coming from the charm quark mass error is $\approx 1-2 \times 10^{-5}$ and is not displayed in Eq. (138), nor is the uncertainty of a similar magnitude coming from higher orders in $\mathrm{G}_{\mathrm{F}}$ expansion. The $e-, \mu$, and $\tau$-lepton contributions in Eq. (42) are evaluated using lepton pole masses and the complete kinematic dependence of $\Pi\left(q^{2}, m_{\ell}\right)$ in Eq. (41). ${ }^{8}$

For $\omega \gg m$, the relative cross-section error is approximately constant, independent of neutrino energy. Relative uncertainties on total cross sections from different sources are summarized in Table II. The dominant uncertainty from the light-quark contribution in differential and absolute cross sections can be expressed as ${ }^{9}$

$$
\begin{aligned}
\delta\left(\frac{\mathrm{d} \sigma_{\mathrm{uds}}^{\nu_{\ell} e \rightarrow \nu_{\ell} e}}{\mathrm{~d} E^{\prime}}\right) \approx & \eta \frac{\mathrm{G}_{\mathrm{F}} m}{\sqrt{2} \pi} \frac{\alpha}{\pi} \hat{\Pi}_{\gamma \gamma}^{(3)}(0) \\
& \times\left|c_{\mathrm{L}}^{\nu_{\ell} e} \mathrm{I}_{\mathrm{L}}+c_{\mathrm{R}} \mathrm{I}_{\mathrm{R}}+\frac{c_{\mathrm{L}}^{\nu_{\ell} e}+c_{\mathrm{R}}}{2} \mathrm{I}_{\mathrm{R}}^{\mathrm{L}}\right|,
\end{aligned}
$$

TABLE II. Relative errors of the total neutrino-electron scattering cross section.

\begin{tabular}{lccc}
\hline \hline & $\begin{array}{c}\text { Light-quark } \\
\text { correction }\end{array}$ & $\begin{array}{c}\text { Effective } \\
\text { couplings }\end{array}$ & $\begin{array}{c}\text { Higher } \\
\text { orders }\end{array}$ \\
\hline$\nu_{\mu} e \rightarrow \nu_{\mu} e\left(X_{\gamma}\right)$ & $0.37 \%$ & $0.068 \%$ & $\lesssim 0.008 \%$ \\
$\bar{\nu}_{\mu} e \rightarrow \bar{\nu}_{\mu} e\left(X_{\gamma}\right)$ & $0.31 \%$ & $0.112 \%$ & $\lesssim 0.005 \%$ \\
$\nu_{e} e \rightarrow \nu_{e} e\left(X_{\gamma}\right)$ & $0.26 \%$ & $0.028 \%$ & $\lesssim 0.007 \%$ \\
$\bar{\nu}_{e} e \rightarrow \bar{\nu}_{e} e\left(X_{\gamma}\right)$ & $0.36 \%$ & $0.044 \%$ & $\lesssim 0.007 \%$ \\
\hline \hline
\end{tabular}

$$
\begin{aligned}
\delta \sigma_{\mathrm{uds}}^{\nu_{\ell} e \rightarrow \nu_{\ell} e} \approx & \eta \frac{\mathrm{G}_{\mathrm{F}} m \omega}{\sqrt{2} \pi} \frac{\alpha}{\pi} \hat{\Pi}_{\gamma \gamma}^{(3)}(0)\left(\frac{2 \omega c_{\mathrm{L}}^{\nu_{\ell} e}}{m+2 \omega}\right. \\
& \left.+\left(1-\frac{m^{3}}{(m+2 \omega)^{3}}\right) \frac{c_{\mathrm{R}}}{3}-\frac{m \omega\left(c_{\mathrm{L}}^{\nu_{\ell} e}+c_{\mathrm{R}}\right)}{(m+2 \omega)^{2}}\right),
\end{aligned}
$$

with the relative uncertainty $\eta=\left(\hat{\Pi}_{3 \gamma}^{(3)}(0) / \hat{\Pi}_{\gamma \gamma}^{(3)}(0)-1.0\right) \approx$ 0.2 and the substitution $c_{\mathrm{L}}^{\nu_{e} e} \leftrightarrow c_{\mathrm{R}}$ in the case of antineutrino scattering.

To illustrate the impact of radiative corrections on the total cross section, Eq. (138) may be compared to the leading-order result of our calculation at scale $\mu=2 \mathrm{GeV}$ and $\omega=1 \mathrm{GeV}$ :

$$
\sigma_{\mathrm{LO}}^{\nu_{\mu} e \rightarrow \nu_{\mu} e}(\omega=1 \mathrm{GeV})=1.5971 \times 10^{-42} \mathrm{~cm}^{2} .
$$

Radiative corrections change the total cross section by $1.7 \%$. We turn now to a discussion of the energy dependence of the radiative corrections.

\section{B. Electron and total electromagnetic energy spectra}

Figures 9 and 10 display the typical size of the radiative corrections to energy spectra with respect to the final electron energy $\left(\bar{E}=E^{\prime}\right)$ and with respect to the total electromagnetic energy (i.e., the electron energy plus photon energy, $\bar{E}=E^{\prime}+k_{\gamma}$ ). We consider muon type neutrinos and antineutrinos, the primary component in the accelerator neutrino beam. In these figures, we show the quantity $\delta$ representing the radiative correction normalized to the leading-order elastic cross section:

\footnotetext{
${ }^{7}$ The error of $\hat{\Pi}_{\gamma \gamma}^{(3)}(0)$ in Eq. (53) contributes \pm 0.00006 .

${ }^{8}$ One can safely evaluate a $\tau$-lepton contribution considering $\Pi\left(0, m_{\tau}\right)$ since $\left|q^{2}\right| \ll m_{\tau}^{2}$.

${ }^{9}$ It can be seen [cf. Eq. (140)] that the muon antineutrino-electron scattering cross section is free from hadronic uncertainty, and also
} effective coupling uncertainty induced by $c_{R}$, at the particular recoil antineutrino energy $\tilde{\omega}$ :

$$
\tilde{\omega}=\frac{\sqrt{\left(c_{\mathrm{L}}^{\nu_{\mu} e}+c_{\mathrm{R}}\right)^{2} m^{2}+8 c_{\mathrm{L}}^{\nu_{\mu} e}\left(c_{\mathrm{L}}^{\nu_{\mu} e}+c_{\mathrm{R}}\right) m \omega-16 c_{\mathrm{L}}^{\nu_{\mu} e} c_{\mathrm{R}} \omega^{2}}-\left(c_{\mathrm{L}}^{\nu_{\mu} e}+c_{\mathrm{R}}\right) m}{4 c_{\mathrm{L}}^{\nu_{\mu} e}} \underset{\omega \gg m}{\longrightarrow} \sqrt{\frac{-c_{\mathrm{R}}}{c_{\mathrm{L}}^{\nu_{\mu}} e}} \omega .
$$




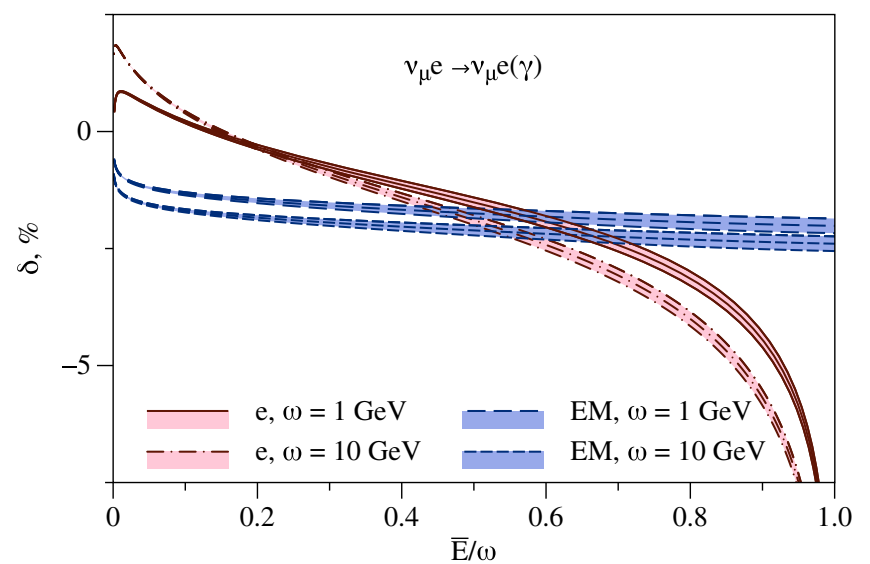

FIG. 9. Radiative corrections to the neutrino-electron scattering process $\nu_{\mu} e \rightarrow \nu_{\mu} e\left(X_{\gamma}\right)$ for two neutrino beam energies $\omega=1$, $10 \mathrm{GeV}$. The quantity $\delta$ is defined in Eq. (143) and strongly depends on the $\overline{\mathrm{MS}}$ scale $\mu$. Three curves for $\mu=\mu_{0} / \sqrt{2}, \mu=\mu_{0}$, and $\mu=\sqrt{2} \mu_{0}$ with $\mu_{0}=2 \mathrm{GeV}$ are presented. The solid and dash-dotted curves correspond with electron spectrum, i.e., $\bar{E}=E^{\prime}$, dashed curves with electromagnetic spectrum, i.e., $\bar{E}=E^{\prime}+k_{\gamma}$. Uncertainties are not shown on this plot with a scale-dependent quantity. Lower curves correspond to a larger value of $\mu$.

$$
\delta=\frac{\mathrm{d} \sigma_{\mathrm{LO}}^{\nu_{\ell} e \rightarrow \nu_{\ell} e \gamma}+\mathrm{d} \sigma_{\mathrm{NLO}}^{\nu_{\ell} e \rightarrow \nu_{\ell} e}-\mathrm{d} \sigma_{\mathrm{LO}}^{\nu_{\ell} e \rightarrow \nu_{\ell} e}}{\mathrm{~d} \sigma_{\mathrm{LO}}^{\nu_{\ell} e \rightarrow \nu_{\ell} e}}
$$

The correction to the electromagnetic energy spectrum is relatively flat over a wide energy, whereas the correction to the electron energy spectrum is logarithmically divergent below the electron end point; cf. Eq. (116). The logarithmic divergence of the electromagnetic energy spectrum above the electron end point [cf. Eq. (133)] is not seen in Fig. 9 due to the small size of the region in Sec. IV I 2 compared to the scale of the figure. Both corrections start from the limit of Eq. (115) at $\bar{E}=m$. Note that the correction $\delta$ depends on the renormalization scale $\mu$ since the numerator does not contain the leading-order elastic process, rather just the virtual correction to it, leaving the scale dependence of the closed fermion loops (Secs. III B and III C) without cancellations. The large renormalization scale dependence in Figs. 9 and 10 illustrates the cancellations occurring between LO and NLO in arriving at the total cross section in Eq. (138). Other uncertainties are not shown in the figure.

\section{Electron angular spectrum}

In this section, we consider the angular smearing of differential cross sections. It can be presented as a function of the variable $X$,

$$
X=2 m\left(1-\frac{\bar{E}}{\omega}\right),
$$

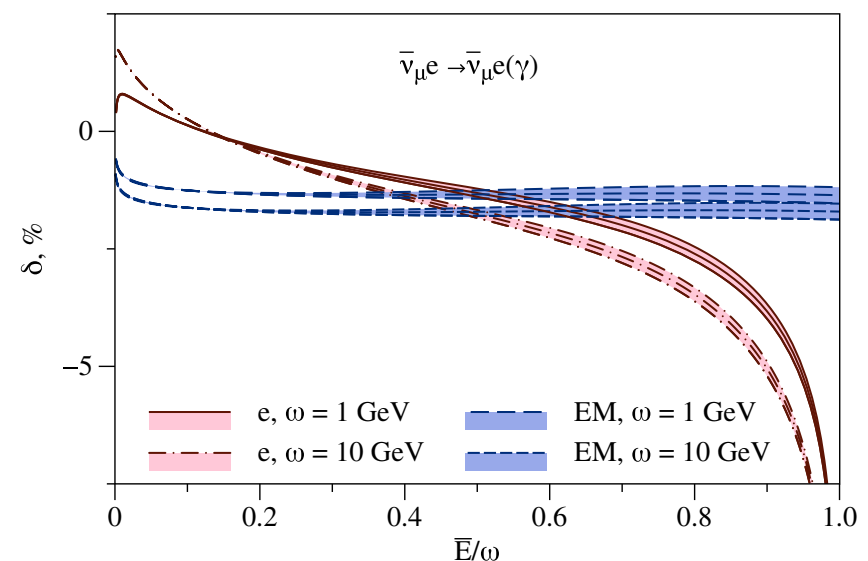

FIG. 10. Same as Fig. 9 for antineutrino-electron scattering process $\bar{\nu}_{\mu} e \rightarrow \bar{\nu}_{\mu} e\left(X_{\gamma}\right)$. Uncertainties are not shown on this plot with a scale-dependent quantity. Lower curves correspond to a large value of $\mu$ for $\bar{E} / \omega \lesssim 0.07-0.1$ and to a smaller value of $\mu$ above.

which becomes $X \approx E^{\prime} \theta_{e}^{2}$ for (anti)neutrinos of high energy in the case of the electron energy spectrum. We present the resulting NLO spectrum in Figs. 11 and 12 for two (anti) neutrino beam energies: $\omega=1 \mathrm{GeV}$ and $10 \mathrm{GeV}$. Although the electromagnetic and electron energy spectra integrate to the same total cross section, shape effects induced by radiative corrections can potentially impact the calibration of neutrino flux. For example, experimental cuts requiring a minimum observed energy will result in different numbers of accepted events depending on which distribution (electromagnetic or electron energy) is chosen. In a practical analysis, neither the electron spectrum nor the electromagnetic spectrum will perfectly represent the experimental

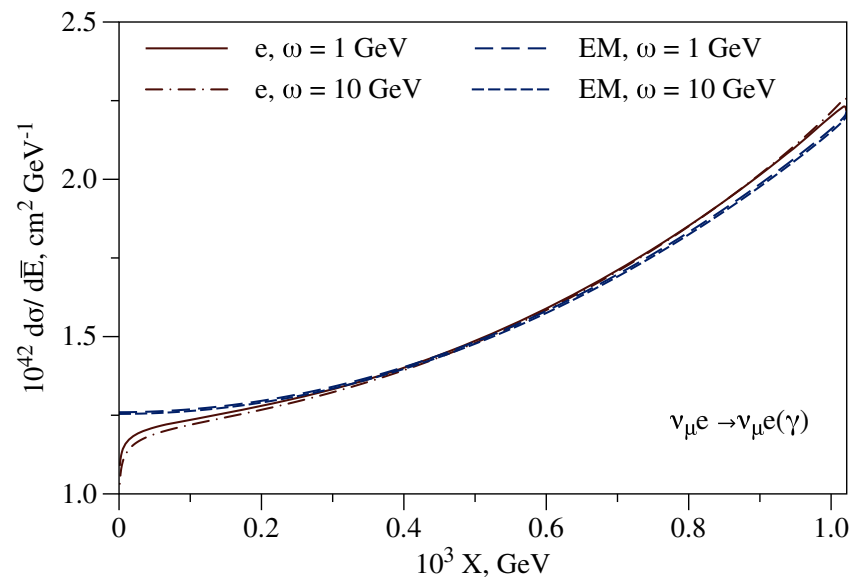

FIG. 11. Energy spectrum in the neutrino-electron scattering $\nu_{\mu} e \rightarrow \nu_{\mu} e(\gamma)$, plotted as a function of $X=2 m(1-\bar{E} / \omega)$ for two neutrino beam energies $\omega=1,10 \mathrm{GeV}$. The solid and dash-dotted curves correspond with the electron spectrum, i.e., $\bar{E}=E^{\prime}$, dashed curves with the electromagnetic spectrum, i.e., $\bar{E}=E^{\prime}+k_{\gamma}$. 


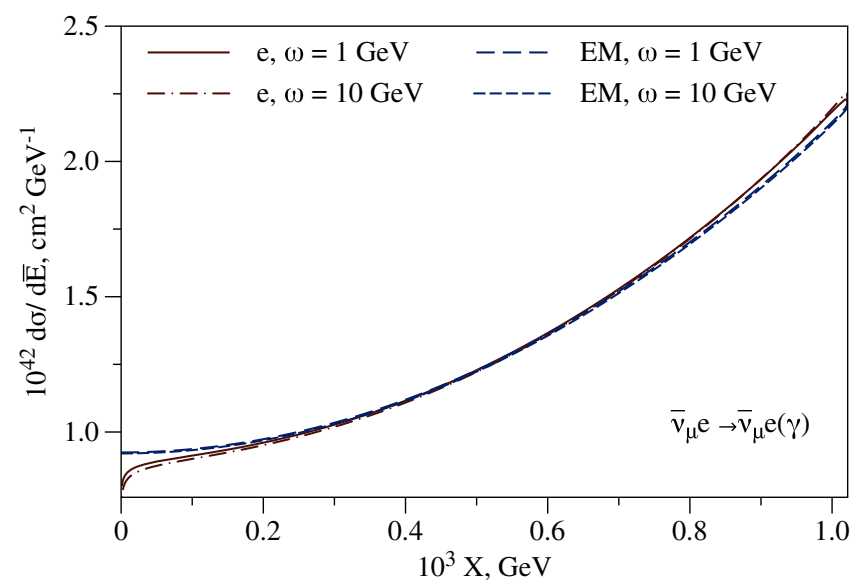

FIG. 12. Same as Fig. 11 for antineutrino-electron scattering process $\bar{\nu}_{\mu} e \rightarrow \bar{\nu}_{\mu} e\left(X_{\gamma}\right)$.

conditions, and the more general distributions presented elsewhere in this paper can be used.

Results comparing $E^{\prime}$ and $E_{\mathrm{EM}}$ distributions after averaging over typical experimental flux profiles are collected in Appendix L.

\section{New physics considerations}

In this section, we consider the impact of radiative corrections on the dynamical zero (22) and isolate the dependence of the neutrino-electron scattering cross section on effective neutrino charge radii. Both effects are present in the Standard Model but may also be used to search for or constrain new physics.

Recall that an amplitude cancellation causes the tree level electron energy spectrum to vanish at the end point of the maximal electron energy, $E_{0}^{\prime}=m+\frac{2 \omega^{2}}{m+2 \omega}$, when electron antineutrinos of a particular energy $\bar{\omega}$ scatter on electrons; cf. Eq. (22). This feature could have implications for novel neutrino oscillation experiments (see, e.g., Refs. [94,95]), and it is thus interesting to determine the impact of radiative corrections on the cancellation. To investigate this question, it is convenient to represent the cross section near the end point as the factorized product of soft and hard functions [121], $d \sigma \sim S H$. The soft function accounts for infrared divergences and real photon emission. Using the explicit forms for virtual corrections from Sec. III, the hard function through first order in $\alpha$ takes the form

$$
H \propto\left(\frac{\omega}{\bar{\omega}}-1\right)\left(\frac{\omega}{\bar{\omega}}-1+\mathcal{O}(\alpha)\right)
$$

For $\omega / \bar{\omega}-1=\mathcal{O}(\alpha)$, the cross section including radiative corrections is suppressed by $\mathcal{O}\left(\alpha^{2}\right)$. The electromagnetic energy spectrum is equal to the electron energy spectrum at tree level and vanishes at the same kinematic point. However, radiative corrections now receive a contribution from "hard" real photon emission, and the electromagnetic spectrum in the vicinity of $\omega=\bar{\omega}$ and $E_{\mathrm{EM}}=E_{0}^{\prime}$ is nonvanishing at first order in $\alpha$. For general $\omega \neq \bar{\omega}$, the electromagnetic energy spectrum vanishes at the end point $E_{\mathrm{EM}}=m+\omega$ and is discontinuous at $E_{\mathrm{EM}}=E_{0}^{\prime}$; at $\omega=\bar{\omega}$ the discontinuity is replaced by a kink.

Neutrino charge radii [122-125] may be systematically defined and computed with low-energy effective field theory [47], where new physics contributions are represented as ${ }^{10}$

$$
\delta c_{\mathrm{L}}^{\nu_{\ell} e}=\delta c_{R}^{\nu_{\ell} e}=\frac{e^{2}}{6} \delta r_{\nu_{\ell}}^{2} .
$$

The impact on neutrino-electron scattering is given by

$\delta\left(\frac{\mathrm{d} \sigma^{\nu_{\ell} e \rightarrow \nu_{\ell} e}}{\mathrm{~d} \bar{E}}\right)=\frac{m \alpha}{3} \delta r_{\nu_{\ell}}^{2}\left|c_{\mathrm{L}}^{\nu_{\ell} e} \mathrm{I}_{\mathrm{L}}+c_{\mathrm{R}} \mathrm{I}_{\mathrm{R}}+\frac{c_{\mathrm{L}}^{\nu_{\ell} e}+c_{\mathrm{R}}}{2} \mathrm{I}_{\mathrm{R}}^{\mathrm{L}}\right|$,

$$
\begin{aligned}
\delta \sigma^{\nu_{\ell} e \rightarrow \nu_{\ell} e}= & \frac{m \omega \alpha}{3} \delta r_{\nu_{\ell}}^{2}\left(\frac{2 \omega c_{\mathrm{L}}^{\nu_{\ell} e}}{m+2 \omega}+\left(1-\frac{m^{3}}{(m+2 \omega)^{3}}\right) \frac{c_{\mathrm{R}}}{3}\right. \\
& \left.-\frac{m \omega\left(c_{\mathrm{L}}^{\nu_{\ell} e}+c_{\mathrm{R}}\right)}{(m+2 \omega)^{2}}\right),
\end{aligned}
$$

with the substitution $c_{\mathrm{L}}^{\nu_{e} e} \leftrightarrow c_{\mathrm{R}}$ in the case of antineutrino scattering.

\section{CONCLUSIONS AND OUTLOOK}

In this work, we have presented analytical results for elastic (anti)neutrino-electron scattering starting from fourfermion effective field theory. Total cross sections, the electron and electromagnetic energy spectra, as well as double- and triple-differential cross sections were presented in a relatively compact form. Our results can be applied to improve constraints of neutrino flux measurements via elastic neutrino-electron scattering. All expressions were obtained for finite electron mass and can also be used in low-energy applications such as oscillation measurements with solar and reactor (anti)neutrinos.

Next-to-leading order corrections with bremsstrahlung of one photon are typically of order few percent and depend on the experimental setup. For instance, as discussed in Sec. VC, electron and electromagnetic energy spectra differ significantly. Although these two spectra integrate to the same total cross section, kinematical cuts can alter

\footnotetext{
${ }^{10}$ In terms of weak scale matching coefficients, this corresponds to a contribution to the neutrino-photon coupling in Ref. [47], $\delta \tilde{c}^{\nu_{\ell} \gamma}=\left(e^{2} / 6\right) \delta r_{\nu_{\ell}}^{2}$. The "charge radius" as a lowenergy observable quantity is unambiguously defined in terms of four-Fermi coefficients in Ref. [47]. For a diagrammatic formulation of neutrino charge radii in the Standard Model see Ref. [126] and references therein.
} 
inferred flux constraints if radiative corrections are not matched correctly to experimental conditions. Future precise measurements of the electron angular spectrum in neutrino-electron scattering can provide energy-dependent neutrino flux constraints. Our results provide a complete description of the kinematic dependence of radiative corrections needed to control uncertainties in neutrino energy reconstruction. We have discussed the impact of radiative corrections on cross sections and energy distributions in searches for physics beyond the Standard Model in Sec. V D.

We provided a complete error budget for neutrinoelectron scattering observables. The light-quark contribution to the radiative correction is the dominant source of uncertainty. We have expressed this contribution in terms of well-defined Standard Model observables, independent of "constituent quark" models used in previous treatments, and determined the relevant hadronic parameter, denoted $\hat{\Pi}_{3 \gamma}^{(3)}(0)$, using $\mathrm{SU}(3)_{f}$ symmetry to relate it to the experimentally constrained parameter $\hat{\Pi}_{\gamma \gamma}^{(3)}(0)$. To further pin down the uncertainty of this light-quark contribution, one can evaluate a closed fermion loop contribution within the dispersion relation approach decomposing $e^{+} e^{-}$crosssection data and measurements of hadronic $\tau$ decays into flavor components $[108,109,112,127-129]$ or perform a calculation in lattice QCD [130,131].

We note that due to the restrictive kinematics of neutrinoelectron scattering $\left(\left|q^{2}\right|<2 m \omega\right.$ for the elastic process) the light-quark contribution enters as a single constant, representing the $q^{2} \rightarrow 0$ limit of the relevant hadronic tensor. This single constant will also impact (and may be constrained by) other low $q^{2}$ processes such as coherent neutrino-nucleus scattering.

Besides its phenomenological relevance, the neutrinoelectron scattering process provides an analytically calculable prototype for the more complicated case of neutrino-nucleus scattering [132]. In general, radiative corrections can be decomposed ("factorized") into soft and hard functions using effective field theory [121]. ${ }^{11}$ The soft functions depend on experimental configuration but are independent of hadronic physics and describe universal large logarithms that are present in general kinematics. The hard functions are independent of experimental configuration and describe hadronic physics. In neutrino-electron scattering the analogous hard functions are perturbatively calculable, whereas in neutrinonucleus scattering they must be parametrized and experimentally constrained.

\section{ACKNOWLEDGMENTS}

We thank K. McFarland for useful discussions. O. T. thanks Matthias Heller for useful discussions regarding radiative corrections in QED. O. T. acknowledges the Fermilab theory group for warm hospitality and support. The work of O. T. is supported by the Visiting Scholars Award Program of the Universities Research Association. The work is supported by the U.S. Department of Energy, Office of Science, Office of High Energy Physics, under Award No. DE-SC0019095 and by the Deutsche Forschungsgemeinschaft DFG through the Collaborative Research Center [The Low-Energy Frontier of the Standard Model (SFB 1044)]. Fermilab is operated by Fermi Research Alliance, LLC under Contract No. DE-AC0207CH11359 with the United States Department of Energy. FEYNCALC [133,134], LOOPTOOLS [135], JAXODRAW [136], Mathematica [137], and DATAGRAPH were extremely useful in this work.

\section{APPENDIX A: QCD CORRECTION TO QED VACUUM POLARIZATION}

For quark loop contributions in Sec. III B, we include the leading QCD correction due to one exchanged gluon inside the quark loop. This correction modifies the form factor $\Pi$ in Eq. (41) as $\Pi \rightarrow \Pi+\Pi^{\mathrm{QCD}}$ with $\Pi^{\mathrm{QCD}}$ from Refs. [104-107] ${ }^{12}$ :

$$
\Pi^{\mathrm{QCD}}=\frac{\alpha_{s}}{3 \pi}\left(\ln \frac{\mu^{2}}{m_{f}^{2}}-4 \zeta(3)+\frac{55}{12}+\frac{4 m_{f}^{2}}{q^{2}} V_{1}\left(\frac{q^{2}}{4 m_{f}^{2}}\right)\right),
$$

where $\alpha_{s}$ is a strong coupling constant, $\zeta(s)$ denotes the Riemann zeta functions, and the function $V(r)$ is given by (for spacelike momentum transfer, $r<0$ )

$$
\begin{aligned}
V(r)= & \sqrt{1-\frac{1}{r}}\left(\frac{8}{3}\left(r+\frac{1}{2}\right)\left(\mathrm{Li}_{2}\left(r_{-}^{2}\right)-\mathrm{Li}_{2}\left(r_{-}^{4}\right)+\ln \frac{-64(1-r)^{2} r}{r_{+}^{3}} \ln r_{+}\right)-2\left(r+\frac{3}{2}\right) \ln r_{+}\right) \\
& +4\left(r-\frac{1}{4 r}\right)\left(2 \mathrm{Li}_{3}\left(r_{-}^{2}\right)-\mathrm{Li}_{3}\left(r_{-}^{4}\right)+\frac{8}{3}\left(\mathrm{Li}_{2}\left(r_{-}^{2}\right)-\mathrm{Li}_{2}\left(r_{-}^{4}\right)\right) \ln r_{+}\right)+\frac{13}{6}+\frac{\zeta(3)}{r} \\
& +\frac{16}{3}\left(r-\frac{1}{4 r}\right) \ln \frac{8(1-r) \sqrt{-r}}{r_{+}^{3}} \ln ^{2} r_{+}-8\left(r-\frac{1}{6}-\frac{7}{48 r}\right) \ln ^{2} r_{+},
\end{aligned}
$$

\footnotetext{
${ }^{11}$ An application of this formalism to the discussion of the dynamical zero in $\bar{\nu}_{e} e$ scattering was described in Sec. (V D).

${ }^{12}$ Note that the color factor applies as $N_{c}\left(\Pi+\Pi^{\mathrm{QCD}}\right)$.
} 
with notations $r_{ \pm}=\sqrt{1-r} \pm r$. As discussed at the end of Sec. III B, the relevant limit for neutrino-electron scattering is $q^{2} \rightarrow-0$, corresponding with

$$
\left.\Pi^{\mathrm{QCD}}\right|_{q^{2} \rightarrow-0}=\frac{\alpha_{s}}{3 \pi}\left(\ln \frac{\mu^{2}}{m_{f}^{2}}+\frac{15}{4}\right) .
$$

For practical evaluation of a $c$-quark contribution, we take the well-convergent expression in terms of $\overline{\mathrm{MS}}$ quark mass $\hat{m}_{c}$ from Refs. [110,138-140],

$$
\begin{aligned}
\Pi= & \frac{1}{3} \ln \frac{\mu^{2}}{\hat{m}_{c}^{2}}+\frac{\alpha_{s}}{3 \pi}\left(-\ln \frac{\mu^{2}}{\hat{m}_{c}^{2}}+\frac{13}{12}\right) \\
& +\frac{\alpha_{s}^{2}}{3 \pi^{2}}\left(\frac{655}{144} \zeta(3)-\frac{3847}{864}-\frac{5}{6} \ln \frac{\mu^{2}}{\hat{m}_{c}^{2}}-\frac{11}{8} \ln ^{2} \frac{\mu^{2}}{\hat{m}_{c}^{2}}\right. \\
& \left.+n_{f}\left(\frac{361}{1296}-\frac{1}{18} \ln \frac{\mu^{2}}{\hat{m}_{c}^{2}}+\frac{1}{12} \ln ^{2} \frac{\mu^{2}}{\hat{m}_{c}^{2}}\right)\right),
\end{aligned}
$$

where $n_{f}=4$ denotes the number of active quarks. The correction of order $\alpha_{s}^{2}$ in Eq. (A4) does not change our results within significant digits.

\section{APPENDIX B: TRIPLE-DIFFERENTIAL DISTRIBUTION}

We evaluate the bremsstrahlung cross section following Ref. [22]. For the electron angle distributions, we introduce the four-vector $l$,

$$
l=k+p-p^{\prime}=\left(l_{0}, \vec{f}\right)
$$

with the laboratory frame values,

$$
\begin{gathered}
l_{0}=m+\omega-E^{\prime} \\
f^{2}=|\vec{f}|^{2}=\omega^{2}+\beta^{2} E^{\prime 2}-2 \omega \beta E^{\prime} \cos \theta_{e} .
\end{gathered}
$$

Note the difference between the electron scattering angle in the elastic process $\left[\Theta_{e}\right.$ of Eq. (6)] and in the scattering with radiation $\left(\theta_{e}\right)$.

The triple-differential cross section with respect to the electron angle, electron energy, and photon energy is given by the following substitutions in Eqs. (58) and (59):

$$
\begin{aligned}
\tilde{\mathrm{I}}_{\mathrm{L}} \rightarrow & \left(\frac{l^{2} f^{2}\left(\left(m+\rho k_{\gamma}\right) l^{2}-2 \rho m^{2} k_{\gamma}\right)}{4 \sqrt{d}}-\frac{m \omega f^{2}\left(\left(m+\rho k_{\gamma}\right)\left(\rho\left(l^{2}-2 m l_{0}\right)-2 m^{2}\right)+2 \rho m^{3}\right)}{\rho \sqrt{d}}\right. \\
& +\frac{\left(\rho m\left(k_{\gamma}^{2}-m^{2}\right)-\rho\left(m+k_{\gamma}\right)\left(l^{2}-2 m l_{0}+m^{2}\right)+\left(2 m+3 k_{\gamma}\right) m^{2}\right) \sigma}{8 \rho^{2} m k_{\gamma} f^{2}}-\frac{\left(m+k_{\gamma}\right) \sigma^{2}}{32 \rho^{2} m k_{\gamma} f^{4}} \\
& -\frac{(1-\rho) m\left(m+k_{\gamma}\right) l^{2}\left(l^{2}-4 k_{\gamma} l_{0}+4 k_{\gamma}^{2}\right)\left(\rho(m+\omega) l^{2}+(1-\rho) m(m+\omega)^{2}-(1+\rho) m \omega^{2}\right)}{16 \rho^{2} k_{\gamma} m f^{4}} \\
& -\frac{\left(l^{2}-2 m \omega\right)^{2}}{8 k_{\gamma}}+\frac{k_{\gamma}\left(l^{2}-m(m+2 \omega)\right)}{2}-\frac{\rho l^{4}-2 m(2 \rho \omega+(2+\rho) m) l^{2}+8 m^{2} \omega\left(\rho l_{0}+2 m\right)}{8 \rho m} \\
& \left.-\frac{\rho^{2} k_{\gamma} m^{4} \omega^{2} f^{4} \sigma}{d^{3 / 2}}-\frac{l^{6}\left(m+k_{\gamma}\right)\left(l^{2}-4 k_{\gamma} l_{0}+4 k_{\gamma}^{2}\right)}{64 k_{\gamma} m f^{4}}\right) \mathcal{D}_{m}, \\
\tilde{\mathrm{I}}_{\mathrm{R}} \rightarrow & \left(\frac{f^{2}\left(\left(\rho k_{\gamma}+m\right)\left(l^{2}-2 m l_{0}\right)^{2}+4 m^{2}\left(\rho k_{\gamma}\left(\left(l_{0}-k_{\gamma}\right)^{2}-m\left(2 l_{0}-k_{\gamma}\right)\right)+m\left(l_{0}-k_{\gamma}\right)^{2}\right)\right)}{4 \sqrt{d}}\right. \\
& +\frac{\rho m^{2} f^{2} l^{2} k_{\gamma}}{2 \sqrt{d}}-\frac{\left(l^{2}-2 m l_{0}\right)^{2}}{8 k_{\gamma}}-\frac{l^{4}}{8 m}-\frac{\rho^{2} k_{\gamma} m^{4} f^{4}\left(l_{0}-k_{\gamma}\right)^{2} \sigma}{d^{3 / 2}}+\frac{\left(2 l_{0}-m\right) l^{2}}{4} \\
& \left.+\frac{1}{2} k_{\gamma} m\left(2 l_{0}-k_{\gamma}-m\right)-m l_{0}\left(l_{0}-m\right)\right) \mathcal{D}_{m}, \\
& \left.+\frac{\rho^{2} k_{\gamma}^{2} m^{5} f^{4} \sigma}{d^{3 / 2}}+\frac{m^{3}\left(l_{0}^{2}+f^{2}+(\rho-1) m l_{0}\right)}{2 \rho f^{2}}\right) \mathcal{D}_{m}, \\
\tilde{\mathrm{I}}_{\mathrm{R}}^{\mathrm{L}} \rightarrow & \left(\frac{m\left(m\left((1-\rho)\left(4 m^{2} l_{0}^{2}-2 m\left(l_{0}+m\right) l^{2}\right)-\rho l^{4}\right)+4 k_{\gamma} \rho\left(-f^{2}-m^{2}+\frac{m l_{0}}{2}\right) l^{2}\right)}{8 \rho k_{\gamma} f^{2}}\right. \\
& -\frac{f^{2} m\left(\rho^{2}\left(l^{2}+m^{2}-2 m k_{\gamma}\right)\left(l^{2}-m^{2}\right)+4 \rho k_{\gamma} m^{3}+(2-\rho)^{2} m^{4}\right)}{\rho(1-\rho) k_{\gamma} m^{6} f^{4} \sigma}+\frac{\rho}{d^{3}}
\end{aligned}
$$


with the kinematical notations

$\sigma=\rho\left(\omega^{2}-f^{2}-E^{2}+m^{2}\right)\left(l^{2}-2 k_{\gamma} l_{0}\right)+4 k_{\gamma} m f^{2}$,

$d=\beta^{2} m^{2} l^{2} \omega^{2}\left(l^{2}+4 k_{\gamma}^{2}-4 k_{\gamma} l_{0}\right) \sin ^{2} \theta_{e}+\frac{\sigma^{2}}{4}$,

where the phase-space factor $\mathcal{D}_{m}$ is given by

$$
\mathcal{D}_{m}=\frac{\pi^{2}}{m^{2} \omega^{2}} \mathrm{~d} f \frac{\mathrm{d} k_{\gamma}}{k_{\gamma}} \frac{\mathrm{d} E^{\prime}}{\omega} .
$$

The physical region of variables corresponding to the radiation of hard photons with energy $k_{\gamma} \geq \varepsilon(\varepsilon \ll m, \omega)$, is the following (see Sec. IV $\mathrm{H}$ for a description of hardand soft-photon regions):

$$
m+\frac{2 \varepsilon^{2}}{m-2 \varepsilon} \leq E^{\prime} \leq m+\frac{2(\omega-\varepsilon)^{2}}{m+2(\omega-\varepsilon)},
$$

$$
\begin{gathered}
|\omega-| \vec{p}^{\prime}|| \leq f \leq l_{0}-2 \varepsilon, \\
\frac{l_{0}-f}{2} \leq k_{\gamma} \leq \frac{l_{0}+f}{2} .
\end{gathered}
$$

We keep the exact dependence on the unphysical parameter $\varepsilon$ which is important in the evaluation of the electron energy spectrum in Sec. IV H. Our integration region in Eqs. (B10)-(B12) corresponds to region $\mathrm{I}$ in Sec. IV H.

\section{APPENDIX C: DOUBLE-DIFFERENTIAL DISTRIBUTION IN ELECTRON ENERGY AND ELECTRON ANGLE}

Integrating Eqs. (B4)-(B6) over the photon energy $k_{\gamma}$, we obtain the double-differential cross section with respect to the recoil electron energy and angle. The result is given by the following substitutions in Eqs. (58) and (59):

$$
\tilde{\mathrm{I}}_{i} \rightarrow \frac{\pi^{2} m}{\omega^{3}}\left(a_{i}+b_{i} \ln \frac{1+\beta}{1-\beta}+c_{i} \ln \frac{l_{0}+f}{l_{0}-f}+d_{i} \ln \frac{l_{0}-\beta f \cos \delta-\sqrt{g}}{l_{0}-\beta f \cos \delta+\sqrt{g}}\right) \mathrm{d} f \mathrm{~d} E^{\prime}-\frac{\pi^{2}}{\omega} \frac{2}{\beta}\left(\beta-\frac{1}{2} \ln \frac{1+\beta}{1-\beta}\right) \mathrm{I}_{i} \frac{\mathrm{d} f^{2}}{l^{2}} \mathrm{~d} E^{\prime},
$$

with $g=\left(f \cos \delta-\beta l_{0}\right)^{2}+\rho^{2} f^{2} \sin ^{2} \delta$ and the angle $\delta$ between vectors $\vec{l}$ and $\vec{p}^{\prime}$,

$$
\cos \delta=\frac{\omega^{2}-\beta^{2} E^{\prime 2}-f^{2}}{2 \beta E^{\prime} f} .
$$

Kinematical factors $\mathrm{I}_{\mathrm{L}}, \mathrm{I}_{\mathrm{R}}, \mathrm{I}_{\mathrm{R}}^{\mathrm{L}}$ in Eq. (C1) correspond to the $2 \rightarrow 2$ process.

The coefficients in integrals $\tilde{\mathrm{I}}_{i}$ are given by

$$
\begin{aligned}
a_{\mathrm{L}}= & \frac{f(2 \omega-m)}{2 m^{2}}-\frac{\beta \cos \delta\left(\frac{3}{4} l^{2}-\frac{f^{2}}{2 \rho}-E^{\prime} l_{0}-2 m \omega\right)}{\rho m^{2}}-\frac{\left(1+\beta^{2} \cos ^{2} \delta\right) f\left(l_{0}+2 m\right)}{4 \rho^{2} m^{2}} \\
& -\frac{\beta^{2} l^{2}\left(1-3 \cos ^{2} \delta\right)\left(l_{0}-4 m\right)}{8 \rho^{2} m^{2} f}-\frac{\left(f-\beta l_{0} \cos \delta\right)\left(l^{2}-m\left(\frac{5}{2} l_{0}-m+3 E^{\prime}\right)\right)}{2 \rho m^{3}}, \\
b_{\mathrm{L}}= & \frac{f l^{2}}{4 \beta m^{3}}-\frac{\omega f}{\beta m^{2}}, \\
c_{\mathrm{L}}= & \frac{\beta^{2} l^{2}\left(1-3 \cos ^{2} \delta\right)\left(l^{2}-4 l_{0} m\right)}{16 \rho^{2} f^{2} m^{2}}-\frac{l^{2}\left(l^{2}-2 s\right)}{8 m^{4}}-\frac{\omega(m+\omega)}{m^{2}} \\
& -\frac{\beta \cos \delta\left(l^{2}\left(l^{2}-4 l_{0} m-m E^{\prime}+m^{2}\right)+4 m^{2} \omega l_{0}\right)}{4 \rho f m^{3}}, \\
d_{\mathrm{L}}= & -\frac{\rho f\left(\left(l^{2}-s\right)^{2}+s\left(s-2 m^{2}\right)\right)}{8 \sqrt{g} m^{4}}-\frac{f \rho \omega}{2 \sqrt{g} m}, \\
a_{\mathrm{R}}= & \frac{3 \beta^{2} \rho^{2} f^{3} l^{2} \sin ^{2} \delta\left(\frac{l_{0}-\beta f \cos \delta}{4 m \rho}+1\right)}{2 g^{2} m}-\frac{f\left(l^{2}-\frac{11 l_{0} m}{2}+m^{2}\right)}{2 m^{3}}+\frac{\beta^{2} f\left(2 f^{2}+3 l_{0}^{2}\right)}{2 g m} \\
& +\frac{f^{2} \rho\left(\beta \cos \delta\left(f^{2}+2 l_{0}\left(\omega-6 E^{\prime}\right)\right)+f\left(2 m-3 l_{0}\right)\right)}{4 g m^{2}}, \\
b_{\mathrm{R}}= & \frac{l^{2} f}{4 \beta m^{3}}-\frac{l_{0} f}{\beta m^{2}},
\end{aligned}
$$




$$
\begin{aligned}
c_{\mathrm{R}}= & -\frac{l^{2}\left(l^{2}-4 m\left(l_{0}-\frac{m}{2}\right)\right)}{8 m^{4}}-\frac{l_{0}\left(l_{0}-m\right)}{m^{2}}, \\
d_{\mathrm{R}}= & \frac{\beta f l^{2}\left(\left(2 l_{0} \rho-m\left(-2 \rho^{2}+\rho+1\right)\right)\left(\beta l_{0}-f \cos \delta\right)-\frac{1}{2} \beta \rho l^{2}\right)}{4 g^{3 / 2} m^{2}}-\frac{\rho^{2} f l_{0}\left(f\left(f-\beta l_{0} \cos \delta\right)+\frac{1}{2} \beta^{2} l^{2}\right)}{g^{3 / 2} m} \\
& -\frac{\rho f\left(l^{2}\left(l^{2}-4 l_{0} m+2 m^{2}\right)+8 l_{0} m^{2}\left(\omega-2 E^{\prime}\right)\right)}{8 \sqrt{g} m^{4}}+\frac{3 \beta^{2} \rho^{3} f^{3} l^{2} \sin ^{2} \delta\left(l^{2}+4 E^{\prime}\left(l_{0}-\beta f \cos \delta\right)\right)}{16 g^{5 / 2} m^{2}}, \\
a_{\mathrm{R}}^{\mathrm{L}}= & \frac{\beta f\left(\beta l_{0}-f \cos \delta\right)}{g}-\frac{\beta \cos \delta}{\rho}, \\
b_{\mathrm{R}=}^{\mathrm{L}=} & -\frac{\rho f l^{2}}{2 \beta m^{3}}, \\
c_{\mathrm{R}}^{\mathrm{L}}= & \frac{\beta l_{0} \cos \delta}{2 \rho f}-\frac{l^{2}-m\left(m+E^{\prime}\right)}{2 m^{2}}, \frac{\rho^{2} f^{2}\left(f-\beta l_{0} \cos \delta\right)}{2 g^{3 / 2}} . \\
d_{\mathrm{R}}^{\mathrm{L}}= & \frac{\rho f\left(\left(m+2 E^{\prime}\right) m-l^{2}\right)}{2 m^{2} \sqrt{g}}-\frac{\rho^{2}}{2}
\end{aligned}
$$

\section{APPENDIX D: DOUBLE-DIFFERENTIAL DISTRIBUTION IN ELECTROMAGNETIC ENERGY AND ELECTRON ANGLE}

To obtain neutrino energy (equivalently, electromagnetic energy) and electron angle distribution, Eqs. (B4)-(B6) can be integrated over the electron energy, exploiting the energy conservation: $k_{\gamma}=m+\omega-\omega^{\prime}-E^{\prime}$. The integration measure of Eq. (B9) is replaced as

$\mathcal{D}_{m}=\frac{\pi^{2}}{m^{2} \omega^{2}} \mathrm{~d} f \frac{\mathrm{d} k_{\gamma}}{k_{\gamma}} \frac{\mathrm{d} E^{\prime}}{\omega} \rightarrow \frac{\pi^{2}}{m^{2} \omega^{2}} \mathrm{~d} \cos \theta_{e} \frac{\mathrm{d} \omega^{\prime}}{k_{\gamma}} \frac{\beta E^{\prime} \mathrm{d} E^{\prime}}{f}$.

The physical integration region is contained in

$$
\begin{gathered}
0 \leq \omega^{\prime} \leq \omega, \\
0 \leq \cos \theta_{e} \leq 1 \\
m \leq E^{\prime} \leq m \frac{(m+\omega)^{2}+\omega^{2} \cos ^{2} \theta_{e}}{(m+\omega)^{2}-\omega^{2} \cos ^{2} \theta_{e}},
\end{gathered}
$$

which is actually larger than the physical region. The extraneous regions I and II are above the electron end point $\left(E_{\mathrm{EM}} \geq E_{0}^{\prime}=m+\frac{2 \omega^{2}}{m+2 \omega}\right)$ and below it $\left(E_{\mathrm{EM}} \leq E_{0}^{\prime}=m+\right.$ $\left.\frac{2 \omega^{2}}{m+2 \omega}\right)$ :

$$
\text { region } \mathrm{I}: 0 \leq \omega^{\prime} \leq \frac{m \omega}{m+2 \omega}
$$

$$
\begin{gathered}
\frac{2 \sqrt{\omega^{\prime}\left(m-\omega^{\prime}\right)\left(\omega-\omega^{\prime}\right)\left(m+\omega-\omega^{\prime}\right)}}{m \omega} \leq \cos \theta_{e} \leq 1, \\
E_{-}^{\prime}\left(\omega^{\prime}\right) \leq E^{\prime} \leq E_{+}^{\prime}\left(\omega^{\prime}\right),
\end{gathered}
$$

$$
\begin{gathered}
\text { region } \mathrm{II}: \frac{m \omega}{m+2 \omega} \leq \omega^{\prime} \leq \omega, \\
\frac{m+\omega}{\omega} \sqrt{\frac{\omega-\omega^{\prime}}{2 m+\omega-\omega^{\prime}}} \leq \cos \theta_{e} \leq 1, \\
E_{-}^{\prime}\left(\omega^{\prime}\right) \leq E^{\prime} \leq m \frac{(m+\omega)^{2}+\omega^{2} \cos ^{2} \theta_{e}}{(m+\omega)^{2}-\omega^{2} \cos ^{2} \theta_{e}}
\end{gathered}
$$

Here $E_{ \pm}^{\prime}\left(\omega^{\prime}\right)$ stand for two solutions $\left(E_{+} \geq E_{-}\right)$of

$$
\begin{aligned}
& \cos \theta_{e} \\
& =\frac{E^{\prime}\left(m+\omega-2 \omega^{\prime}\right)-m^{2}+m\left(2 \omega^{\prime}-\omega\right)+2 \omega^{\prime}\left(\omega-\omega^{\prime}\right)}{\omega \sqrt{E^{\prime 2}-m^{2}}} .
\end{aligned}
$$

The presentation here in terms of a larger region (D2)(D4) and subtractions (D5)-(D10) is designed as a simple description of the actual physical region. In practice, one may perform the integration over this larger region and use subtractions above the electron end point $E_{\mathrm{EM}} \geq E_{0}^{\prime}=$ $m+\frac{2 \omega^{2}}{m+2 \omega}$; or one may break up the integration region (D2)-(D4) and integrate once only over the physical region.

\section{APPENDIX E: DOUBLE-DIFFERENTIAL DISTRIBUTION IN PHOTON ENERGY AND ELECTRON ENERGY}

To obtain the distribution with respect to the photon energy and electron energy, Eqs. (B4)-(B6) can be integrated first over the variable $f$ after the change of the integration order. The kinematical region of electron energy is bounded as 


$$
m \leq E^{\prime} \leq m+\frac{2 \omega^{2}}{m+2 \omega} .
$$

The physical region of $f$ for different values of $k_{\gamma}$ is then given by

$$
\begin{gathered}
0 \leq k_{\gamma} \leq \frac{l_{0}-|\omega-| \vec{p}^{\prime}||}{2}, \quad l_{0}-2 k_{\gamma} \leq f \leq l_{0} ; \quad \text { (E2) } \\
\frac{l_{0}-|\omega-| \vec{p}^{\prime}||}{2} \leq k_{\gamma} \leq \frac{l_{0}+|\omega-| \vec{p}^{\prime} \mid}{2}, \quad|\omega-| \vec{p}^{\prime}|| \leq f \leq l_{0} ; \\
\frac{l_{0}+|\omega-| \vec{p}^{\prime}||}{2} \leq k_{\gamma} \leq l_{0}, \quad-l_{0}+2 k_{\gamma} \leq f \leq l_{0} . \quad \text { (E4) } \\
\text { APPENDIX F: DOUBLE-DIFFERENTIAL } \\
\text { DISTRIBUTION IN PHOTON ENERGY AND } \\
\text { PHOTON ANGLE }
\end{gathered}
$$

\section{APPENDIX F: DOUBLE-DIFFERENTIAL DISTRIBUTION IN PHOTON ENERGY AND PHOTON ANGLE}

We evaluate the bremsstrahlung cross section with respect to the photon energy and photon angle considering the final photon energy spectrum instead of the electron spectrum [22]; see Sec. IV D for explanations. For the photon scattering angle (with respect to the neutrino beam direction) distributions, we introduce the four-vector $\bar{l}$,

$$
\bar{l}=k+p-k_{\gamma}=\left(\bar{l}_{0}, \overrightarrow{\bar{f}}\right)
$$

with the laboratory frame values,

$$
\begin{gathered}
\bar{l}_{0}=m+\omega-k_{\gamma}, \\
\bar{f}^{2}=|\overrightarrow{\bar{f}}|^{2}=\omega^{2}+k_{\gamma}^{2}-2 \omega k_{\gamma} \cos \theta_{\gamma},
\end{gathered}
$$

where $\theta_{\gamma}$ denotes the photon scattering angle.

The photon energy spectrum accounting for electron mass terms is given by the following substitutions in Eqs. (58) and (59):

$$
\tilde{\mathrm{I}}_{i} \rightarrow \frac{\pi^{2}}{2 m \omega^{2}}\left(a_{i}\left(\bar{l}^{2}-m^{2}\right)+b_{i} \ln \frac{m^{2}}{\bar{l}^{2}}\right) \frac{\bar{f} \mathrm{~d} \bar{f}}{\left(\bar{l}^{2}-s\right)^{2}} \mathrm{~d} k_{\gamma},
$$

with $s=m^{2}+2 m \omega$ and coefficients $a_{i}$ and $b_{i}$ in Eq. (F4):

$$
\begin{aligned}
a_{\mathrm{L}}= & \frac{\left(\bar{l}^{2}-m^{2}\right)^{2}\left(2 \bar{l}^{2}\left(k_{\gamma} \bar{l}_{0}+m\left(2 \bar{l}_{0}-m\right)\right)+m\left(-2 \bar{l}_{0}^{2}(2 \omega+m)+\bar{l}_{0} m(6 \omega+m)-3 m^{2} \omega\right)\right)}{4 k_{\gamma}^{2} \bar{l}^{2} m \omega} \\
& -\frac{4 m \omega^{3}\left(m\left(2 k_{\gamma}-\omega+m\right)+\bar{l}^{2}\right)-\omega\left(\omega-k_{\gamma}\right)\left(3 \bar{l}^{4}-6 \bar{l}^{2} \bar{l}_{0} m-m^{3}\left(2 \bar{l}_{0}-5 m\right)\right)}{k_{\gamma}^{2} \bar{l}^{2}} \\
& -\frac{2 \omega^{2}\left(\bar{l}^{4}-\bar{l}^{2} m\left(5 \bar{l}_{0}-3 m\right)+m^{2}\left(2 \bar{l}_{0}-3 m\right)\left(\bar{l}_{0}-2 m\right)\right)}{k_{\gamma}^{2} \bar{l}^{2}}-\frac{\left(\bar{l}^{2}-m^{2}\right)^{2} \bar{l}^{2}\left(k_{\gamma}+m\right)}{4 k_{\gamma}^{2} m^{2} \omega}, \\
\frac{a_{\mathrm{R}} /\left(\bar{l}^{2}-m^{2}\right)}{\bar{f}^{2}-\left(\bar{l}_{0}-m\right)^{2}}= & -\frac{m\left(\bar{l}^{2}-s\right)\left(-4 k_{\gamma}^{3}(11 \omega+17 m)-4 k_{\gamma}^{2}\left(35 \omega^{2}+103 m^{2}\right)-3 k_{\gamma} m^{2}(12 \omega+29 m)\right)}{12 k_{\gamma}^{2} \bar{l}^{6} \omega} \\
& -\frac{2 m^{3} s\left(k_{\gamma}^{2}\left(344 \omega^{2}+1116 m \omega+537 m^{2}\right)+m\left(312 \omega^{3}+501 m \omega^{2}+342 m^{2} \omega+72 m^{3}\right)\right)}{3 \bar{l}^{6} \omega\left(\bar{l}^{2}-m^{2}\right)\left(\bar{f}^{2}-\left(\bar{l}_{0}-m\right)^{2}\right)} \\
& -\frac{4 m^{2} s^{2}\left(6 k_{\gamma}^{2} m\left(27 \omega^{2}+93 m \omega+46 m^{2}\right)+k_{\gamma} s\left(51 \omega^{2}+154 m \omega+108 m^{2}\right)-3 s m \omega^{2}\right)}{3 \bar{l}^{6} \omega\left(\bar{l}^{2}-m^{2}\right)\left(\bar{l}^{2}-s\right)\left(\bar{f}^{2}-\left(\bar{l}_{0}-m\right)^{2}\right)} \\
& -\frac{m^{3}\left(\bar{l}^{2}-s\right)\left(4 k_{\gamma}^{2}\left(64 \omega^{2}+197 m \omega+96 m^{2}\right)+k_{\gamma} m\left(2950 \omega^{2}+3376 m \omega+1191 m^{2}\right)\right)}{3 \bar{l}^{6} \omega\left(\bar{l}^{2}-m^{2}\right)\left(\bar{f}^{2}-\left(\bar{l}_{0}-m\right)^{2}\right)} \\
& -\frac{m^{2}\left(\bar{l}^{2}-s\right)\left(-4 k_{\gamma}^{2} \omega+k_{\gamma}\left(268 \omega^{2}+794 m \omega+384 m^{2}\right)+2 m^{2}(785 \omega+327 m)\right)}{6 \bar{l}^{6} \omega\left(\bar{f}^{2}-\left(\bar{l}_{0}-m\right)^{2}\right)} \\
& -\frac{2 m^{2} s\left(k_{\gamma}^{2} m\left(590 \omega^{3}+2106 m \omega^{2}+2144 m^{2} \omega+617 m^{3}\right)+92 k_{\gamma} m \omega^{4}-2 s^{2} \omega^{2}\right)}{3 k_{\gamma} \bar{l}^{6} \omega\left(\bar{l}^{2}-m^{2}\right)\left(\bar{f}^{2}-\left(\bar{l}_{0}-m\right)^{2}\right)} \\
& -\frac{m^{2}\left(\bar{l}^{2}-s\right)\left(2 \omega^{2} k_{\gamma}(162 \omega+579 m)-m\left(16 \omega^{3}-18 m \omega^{2}-105 m^{2} \omega-64 m^{3}\right)\right)}{6 k^{6} \omega\left(\bar{f}^{2}-\left(\bar{l}_{0}-m\right)^{2}\right)} \\
&
\end{aligned}
$$




$$
\begin{aligned}
& -\frac{4 m^{3}\left(\bar{l}^{2}-s\right)\left(193 k_{\gamma}^{2} \omega^{3}-m\left(4 \omega^{2}+2 m \omega-3 m^{2}\right)\left(\omega^{2}+m \omega+m^{2}\right)\right)}{3 k_{\gamma} \bar{l}^{6} \omega\left(\bar{l}^{2}-m^{2}\right)\left(\bar{f}^{2}-\left(\bar{l}_{0}-m\right)^{2}\right)}+\frac{11\left(\bar{l}^{2}-m^{2}\right)}{6 \bar{l}^{4}} \\
& -\frac{m^{3}\left(\bar{l}^{2}-s\right)\left(184 \omega^{4}+740 m \omega^{3}+1344 m^{2} \omega^{2}+1167 m^{3} \omega+405 m^{4}\right)}{3 \bar{l}^{6} \omega\left(\bar{l}^{2}-m^{2}\right)\left(\bar{f}^{2}-\left(\bar{l}_{0}-m\right)^{2}\right)}+\frac{106 m^{2}\left(\bar{l}^{2}-s\right)}{3 \bar{l}^{6}} \\
& +\frac{\left(\bar{l}^{2}-m^{2}\right)\left(k_{\gamma}^{2}+11 k_{\gamma} m+\omega^{2}+6 m^{2}\right)}{3 k_{\gamma} \bar{l}^{4} \omega}+\frac{\left(k_{\gamma}+m\right)\left(\bar{l}^{2}-m^{2}\right)\left(\bar{f}^{2}-\left(\bar{l}_{0}-m\right)^{2}\right)^{2}}{12 k_{\gamma}^{2} m^{2} \omega\left(\bar{l}^{2}-s\right)^{2}} \\
& -\frac{8 k_{\gamma} m^{3} s^{3}\left(48 k_{\gamma} s+\omega^{2}\left(27 k_{\gamma}-m\right)\right)}{3 \bar{l}^{6} \omega\left(\bar{l}^{2}-m^{2}\right)\left(\bar{l}^{2}-s\right)^{2}\left(\bar{f}^{2}-\left(\bar{l}_{0}-m\right)^{2}\right)}-\frac{1}{6 k_{\gamma}^{2}}-\frac{m^{5}\left(\bar{l}^{2}-s\right)}{12 k_{\gamma}^{2} \bar{l}^{6} \omega}, \\
& a_{\mathrm{R}}^{\mathrm{L}}=\frac{\bar{l}^{2} m\left(8 k_{\gamma}^{2}+14 k_{\gamma} \bar{l}_{0}-9 k_{\gamma} m-2 \bar{l}_{0} m\right)}{2 k_{\gamma}^{2} \omega}-\frac{m^{4} s^{2}\left(2 k_{\gamma}-m\right)^{2}\left(2 \bar{l}_{0}-m\right)}{4 k_{\gamma}^{2} \bar{l}^{4} \omega\left(\bar{l}^{2}-s\right)} \\
& +\frac{m^{3}\left(8 k_{\gamma}^{3}(2 \omega+5 m)-8 k_{\gamma}^{2}\left(6(\omega+m)^{2}+m(\omega+2 m)\right)+4 k_{\gamma}\left(8(\omega+m)^{3}-3 s \omega\right)\right)}{4 k_{\gamma}^{2} \bar{l}^{2} \omega} \\
& +\frac{m^{2}\left(-4 k_{\gamma}^{3}+2 k_{\gamma}^{2}(8 \omega-7 m)-k_{\gamma}\left(28 \omega^{2}+34 m \omega+15 m^{2}\right)+m\left(3(\omega+m)^{2}-\omega^{2}\right)\right)}{2 k_{\gamma}^{2} \omega} \\
& -\frac{m^{3}\left(8 k_{\gamma}^{2}\left(16 k_{\gamma} \omega+13 k_{\gamma} m+m^{2}\right)-s\left(2 k_{\gamma}-m\right)^{2}+2 k_{\gamma} m\left(4 k_{\gamma}-m\right)^{2}\right)}{4 k_{\gamma}^{2} \omega\left(\bar{l}^{2}-s\right)} \\
& -\frac{\bar{l}^{4}\left(4 k_{\gamma}-m\right)}{4 k_{\gamma}^{2} \omega}-\frac{m^{3} s\left(2 s+3 m^{2}\right)}{4 k_{\gamma}^{2} \bar{l}^{2} \omega}, \\
& b_{\mathrm{L}}=-\left(\bar{l}^{2}-m^{2}\right)^{2}-4 m^{2} \omega(\omega+2 m) \text {, } \\
& \frac{b_{\mathrm{R}}}{\bar{f}^{2}-\left(\bar{l}_{0}-m\right)^{2}}=\frac{16 k_{\gamma} m^{3}(\omega+m)\left((\omega+2 m)^{2}+4 m \omega\right)}{\omega\left(\bar{l}^{2}-s\right)^{2}}+\frac{\bar{l}^{2}\left(k_{\gamma}(\omega+2 m)+m^{2}\right)}{k_{\gamma} \omega} \\
& +\frac{m\left(2 k_{\gamma}^{2}(\omega+m)+(2 \omega+3 m)\left(k_{\gamma}(\omega+4 m)+m^{2}\right)\right)}{k_{\gamma} \omega} \\
& -\frac{8 m^{2}\left(\bar{l}_{0}\left((\omega+2 m)^{2}+m(\omega-m)\right)-2(\omega+m)^{2}(\omega+4 m)\right)}{\omega\left(\bar{l}^{2}-s\right)}, \\
& b_{\mathrm{R}}^{\mathrm{L}}=-\frac{\bar{l}^{4}\left(m^{2}\left(-12 k_{\gamma} \omega+20 \omega(\omega+m)+7 m^{2}\right)-2 \bar{l}^{2} m\left(\bar{l}_{0}+\omega\right)+\left(\bar{l}^{2}-s\right)^{2}\right)}{k_{\gamma} \omega\left(\bar{l}^{2}-s\right)} \\
& -\frac{2 \bar{l}^{2} m^{2}\left(2 k_{\gamma}^{2} m(2 \omega+3 m)+k_{\gamma} m\left(7 m^{2}+10 m \omega+12 \omega^{2}\right)-4 s\left((\omega+m)^{2}+\omega^{2}\right)\right)}{k_{\gamma} \omega\left(\bar{l}^{2}-s\right)} \\
& -\frac{m^{2}\left(4 k_{\gamma}^{2} m^{3}(8 \omega+5 m)-8 k_{\gamma} m s\left((\omega+m)^{2}+m^{2}\right)+s^{2}\left((2 \omega+m)^{2}+2 m^{2}\right)\right)}{k_{\gamma} \omega\left(\bar{l}^{2}-s\right)} .
\end{aligned}
$$

\section{APPENDIX G: PHOTON ENERGY SPECTRUM}

The photon energy spectrum accounting for electron mass terms is given by the following substitutions in Eqs. (58) and (59):

$$
\begin{aligned}
\tilde{\mathrm{I}}_{i} \rightarrow & \frac{\pi^{2}}{\omega^{3}}\left[a_{i}+b_{i} \ln \frac{k_{\gamma}}{\omega}+c_{i} \ln \frac{2 \bar{l}_{0}-m}{m}-d_{i} \ln \frac{2 k_{\gamma}}{2 \omega+m} \ln \frac{2 \bar{l}_{0}-m}{m}\right. \\
& \left.+d_{i} \sum_{\sigma_{1}, \sigma_{2}= \pm} \Re\left(\operatorname{Li}_{2} \frac{\bar{l}_{0}+\sigma_{1} \sqrt{\bar{l}_{0}^{2}-m^{2}}}{\bar{l}_{0}+\sigma_{2} \sqrt{\left(\bar{l}_{0}-m\right)^{2}-2 m k_{\gamma}}}-\mathrm{Li}_{2} \frac{\bar{l}_{0}+\sigma_{1}\left(\bar{l}_{0}-m\right)}{\bar{l}_{0}+\sigma_{2} \sqrt{\left(\bar{l}_{0}-m\right)^{2}-2 m k_{\gamma}}}\right)\right] \mathrm{d} k_{\gamma},
\end{aligned}
$$


with coefficients $a_{i}, b_{i}, c_{i}$, and $d_{i}$ in Eq. (G1):

$$
\begin{aligned}
& a_{\mathrm{L}}=\frac{\left(\omega-k_{\gamma}\right)\left(2 k_{\gamma}^{3}-k_{\gamma}^{2} m+6 k_{\gamma} m^{2}-2 \omega^{2}\left(53 k_{\gamma}+2 m\right)-\omega\left(8 k_{\gamma}-m\right)\left(5 k_{\gamma}+3 m\right)-3 m^{3}\right)}{24 k_{\gamma}^{2}}, \\
& \frac{a_{\mathrm{R}}}{\omega-k_{\gamma}}=\frac{m^{5}}{24 k_{\gamma}^{2}(2 \omega+m)^{2}}+\frac{m^{3}\left(-36 k_{\gamma}^{2}-10 k_{\gamma} m+m^{2}\right)}{96 k_{\gamma}^{3}(2 \omega+m)}-\frac{m\left(k_{\gamma}^{3} m+\left(k_{\gamma}-\frac{m}{4}\right)\left(4 k_{\gamma}^{3}-2 k_{\gamma}^{2} m-m^{3}\right)\right)}{24 k_{\gamma}^{3}\left(2 \omega-2 k_{\gamma}+m\right)} \\
& +\frac{m^{2} \omega}{12\left(2 \omega-2 k_{\gamma}+m\right)^{2}}-\frac{\omega^{2}\left(73 k_{\gamma}+2 m\right)}{36 k_{\gamma}^{2}}-\frac{m(656 \omega+897 m)}{144 k_{\gamma}}-\frac{37 k_{\gamma}}{36}-\frac{892 \omega+1184 m}{144}, \\
& a_{\mathrm{R}}^{\mathrm{L}}=\frac{m^{2} \omega\left(\omega-k_{\gamma}\right)(2 \omega+3 m)}{8 k_{\gamma}^{2}(2 \omega+m)}-\frac{m\left(\omega-k_{\gamma}\right)\left(26 k_{\gamma}^{2}-k_{\gamma}(22 \omega-13 m)+3 m^{2}\right)}{8 k_{\gamma}^{2}}, \\
& b_{\mathrm{L}}=-\frac{\omega^{2}\left(3 k_{\gamma}(2 \omega+m)+2 \omega(\omega+m)\right)}{k_{\gamma}(2 \omega+m)}, \\
& b_{\mathrm{R}}=\frac{4 m \omega^{4}(\omega+m)}{3 k_{\gamma}(2 \omega+m)^{3}}-\frac{6 \omega^{4}}{(2 \omega+m)^{2}}+\frac{\omega^{2}\left(3 k_{\gamma}^{2}+14 \omega^{2}\right)}{3 k_{\gamma}(2 \omega+m)}-\frac{14 \omega^{3}}{3 k_{\gamma}} \\
& -\frac{\omega\left(8 k_{\gamma} m+3 k_{\gamma}\left(k_{\gamma}+\omega\right)-2\left(\omega^{2}-m^{2}\right)\right)}{k_{\gamma}}, \\
& b_{\mathrm{R}}^{\mathrm{L}}=-\frac{m \omega\left(k_{\gamma}(2 \omega+m)(2 \omega+3 m)-2 \omega^{3}+3 m^{2} \omega+m^{3}\right)}{k_{\gamma}(2 \omega+m)^{2}}, \\
& c_{\mathrm{L}}=\frac{m^{2}\left(4 \omega^{2}-3 m^{2}\right)}{16 k_{\gamma}(2 \omega+m)}-\frac{8 k_{\gamma}^{3} \omega+2 k_{\gamma}^{2}\left(4 \omega^{2}-m^{2}\right)-16 k_{\gamma} \omega^{2}(\omega+m)-m^{4}}{16 k_{\gamma}^{2}}, \\
& c_{\mathrm{R}}=-\frac{m^{4}\left(36 \omega^{2}+30 m \omega+7 m^{2}\right)}{24 k_{\gamma}(2 \omega+m)^{3}}+\frac{3 m^{4}}{8(2 \omega+m)^{2}}-\frac{3 m^{3}}{2(2 \omega+m)}-k_{\gamma}\left(\frac{\omega^{2}}{2 \omega+m}+\frac{\omega}{2}+\frac{15 m}{4}\right) \\
& +\frac{\omega^{3}-k_{\gamma}^{3}}{3 k_{\gamma}}+\frac{m\left(72 \omega^{2}+204 m \omega+123 m^{2}\right)}{48 k_{\gamma}}+\frac{m^{4}}{48 k_{\gamma}^{2}}-\frac{13 m^{2}}{8}+\omega(\omega+3 m), \\
& c_{\mathrm{R}}^{\mathrm{L}}=\frac{m \omega\left(8 k_{\gamma}(\omega+m)(2 \omega+m)-\omega\left(8 \omega^{2}+12 m \omega+3 m^{2}\right)\right)}{2 k_{\gamma}(2 \omega+m)^{2}}-\frac{m\left(2 k_{\gamma}-m\right)\left(8 k_{\gamma}\left(k_{\gamma}+m\right)+3 m^{2}\right)}{16 k_{\gamma}^{2}}, \\
& d_{\mathrm{L}}=-\omega^{2}, \\
& d_{\mathrm{R}}=-\frac{k_{\gamma}^{2}(2 \omega+3 m)+2 k_{\gamma}(\omega+2 m)^{2}+2 m^{2}(\omega+m)}{2 k_{\gamma}}, \\
& d_{\mathrm{R}}^{\mathrm{L}}=-\frac{m^{2}\left(3 k_{\gamma}+m\right)}{2 k_{\gamma}} .
\end{aligned}
$$

\section{APPENDIX H: ELECTRON ENERGY SPECTRUM}

The nonfactorizable contribution to the electron energy spectrum $\mathrm{d} \sigma_{\mathrm{NF}}^{\nu_{e} e \nu_{\ell} e \gamma}$ from Eq. (107) is given by the following substitutions in Eqs. (58) and (59):

$$
\begin{aligned}
\tilde{\mathrm{I}}_{i} \rightarrow & \frac{\pi^{2}}{\omega^{3}}\left(z_{i}+y_{i} \ln \frac{\frac{2 \omega}{m}}{-1+\frac{\rho}{1+\beta}\left(1+\frac{2 \omega}{m}\right)}+x_{i} \ln \frac{\frac{2 l_{0}}{m}}{1+\frac{2 \omega}{m}-\frac{1+\beta}{\rho}}+r_{i} \ln \frac{1-\frac{1+\beta}{\rho}}{\frac{1+\beta}{1-\beta}-\frac{1+\beta}{\rho}\left(1+\frac{2 \omega}{m}\right)}\right) \mathrm{d} E^{\prime} \\
& +\frac{\pi^{2}}{\omega^{3}}\left(q_{i} \ln \frac{1+\beta}{1-\beta}+v_{i}\left(\operatorname{Li}_{2} \frac{1+\beta}{\rho}-\operatorname{Li}_{2}\left(1+\frac{2 \omega}{m}\right)+\operatorname{Li}_{2} \frac{\left(1+\frac{2 \omega}{m}\right) \rho}{1+\beta}-\frac{\pi^{2}}{6}\right)\right) \mathrm{d} E^{\prime} .
\end{aligned}
$$

Exact expressions for coefficients $z_{i}, y_{i}, x_{i}, r_{i}, q_{i}$, and $v_{i}$ in Eq. (H1) are given by 


$$
\begin{aligned}
& v_{\mathrm{L}}=\frac{1}{2}\left(\frac{m^{2}}{2}+2 m \omega+\omega^{2}\right), \quad v_{\mathrm{R}}=\frac{1}{2}\left(l_{0}^{2}+\frac{\beta^{2}+\rho}{\rho^{2}} m^{2}\right), \quad v_{\mathrm{R}}^{\mathrm{L}}=\frac{1}{2} m\left(2 l_{0}-m\right), \\
& x_{\mathrm{L}}=-\frac{2}{15} \frac{\omega^{5}}{m^{3}}+\frac{1}{3} \frac{\omega^{3}}{m}+\left(\frac{1+3 \beta^{2}}{3 \rho^{3}}-\frac{4 \beta^{4}-11 \beta^{2}+7}{3 \rho^{4}}\right) \omega^{2}+\left(\frac{2}{\rho^{3}}-\frac{\beta^{4}-\beta^{2}+2}{\rho^{4}}\right) m \omega \\
& +\left(\frac{-7 \beta^{4}+14 \beta^{2}-22}{15 \rho^{4}}+\frac{15 \beta^{4}-25 \beta^{2}+22}{15 \rho^{5}}\right) m^{2} \\
& x_{\mathrm{R}}=-\frac{l_{0}^{2}\left(35 l_{0} m^{2}-10 l_{0}^{2} m+2 l_{0}^{3}-30 m^{3}\right)}{15 m^{3}} \text {, } \\
& x_{\mathrm{R}}^{\mathrm{L}}=\frac{3 l_{0} m^{2}-3 l_{0}^{2} m-2 l_{0}^{3}+3 m^{2} \omega}{3 m}, \\
& y_{\mathrm{L}}=\frac{1}{2} \omega(\omega-m) \text {, } \\
& y_{\mathrm{R}}=\frac{-\omega^{4}-2\left(5-\frac{1}{\rho}\right) m \omega^{3}+\frac{12 \beta^{2}+11 \rho-16}{\rho^{2}} m^{2} \omega^{2}+\frac{6 \beta^{2}+9 \rho-10}{\rho^{2}} m^{3} \omega+\frac{\beta^{2}+2 \rho-2}{\rho^{2}} m^{4}}{(m+2 \omega)^{2}}, \\
& y_{\mathrm{R}}^{\mathrm{L}}=m E^{\prime}\left(1-\frac{(m+2 \omega)^{2}-m \omega}{E^{\prime}(m+2 \omega)}\right) \text {, } \\
& r_{\mathrm{L}}=\left(-\frac{2+\beta}{3} \frac{\rho}{(1+\beta)^{2}}+\frac{1}{6} \frac{4+\beta}{1+\beta}\right) \omega^{2}+\left(\frac{\beta-\rho^{2}}{\rho(1+\beta)}+\frac{1}{2}\left(1+\frac{1}{(1+\beta)^{2}}\right)\right) m \omega \\
& +\left(-\frac{\left(17 \beta^{2}+36 \beta+22\right) \rho}{30(1+\beta)^{3}}+\frac{14 \beta^{2}+43 \beta+44}{60(1+\beta)^{2}}\right) m^{2} \text {, } \\
& r_{\mathrm{R}}=\left(-\frac{2+\beta}{3} \frac{\rho}{(1+\beta)^{2}}+\frac{1}{6} \frac{4+\beta}{1+\beta}\right) \omega^{\prime 2}+\left(\frac{\beta^{2}-5 \beta+1}{3 \rho(1+\beta)}+\frac{1}{6} \frac{7 \beta^{2}+8 \beta-2}{(1+\beta)^{2}}\right) m \omega^{\prime} \\
& +\left(\frac{-23 \beta^{3}+14 \beta^{2}+41 \beta-2}{30 \rho(1+\beta)^{2}}+\frac{-28 \beta \rho^{2}+43 \beta^{2}+2}{30 \rho^{2}(1+\beta)}\right) m^{2}, \\
& r_{\mathrm{R}}^{\mathrm{L}}=\frac{1}{6}\left(14+5 \beta+2 \frac{2 \beta^{2}-4 \beta-7}{\rho}\right) \frac{m^{2}}{1+\beta}+\left(1+\frac{1-2 \rho}{1+\beta}\right) m \omega, \\
& q_{\mathrm{L}}=\left(\frac{1}{2 \beta} \frac{\rho}{1+\beta}-\frac{1+\beta}{2 \beta}\right) \omega^{2}+\frac{\beta}{2 \rho} m \omega+\frac{1-\rho}{2 \beta} m^{2}, \\
& q_{\mathrm{R}}=\left(\frac{1}{2 \beta} \frac{\rho}{1+\beta}-\frac{1+\beta}{2 \beta}\right) \omega^{\prime 2}+\left(2-\frac{1}{1+\beta}-\frac{2-\beta}{2 \rho}\right) m \omega^{\prime} \\
& +\left(\frac{4 \beta^{3}+\beta^{2}-4 \beta+2}{4 \beta \rho^{2}}+\frac{-\beta^{3}+2 \beta^{2}+\beta-1}{2 \beta \rho(1+\beta)}\right) m^{2}, \\
& q_{\mathrm{R}}^{\mathrm{L}}=\frac{(1-\beta) \omega^{2}-2 \rho m \omega+\left(1+\frac{\beta}{2}\right) m^{2}}{\beta} \frac{l_{0}-\omega}{m}+\beta m E^{\prime}, \\
& z_{\mathrm{L}}=\frac{z^{\omega^{4}} \omega^{4}+z_{\mathrm{L}}^{\omega^{3}} m \omega^{3}+z_{\mathrm{L}}^{\omega^{2}} m^{2} \omega^{2}+z_{\mathrm{L}}^{\omega} m^{3} \omega+z_{\mathrm{L}}^{0} m^{4}}{m^{2}}, \\
& z_{\mathrm{R}}=\frac{2 z^{\omega^{4}} \omega^{5}+z_{\mathrm{R}}^{\omega^{4}} m \omega^{4}+z_{\mathrm{R}}^{\omega^{3}} m^{2} \omega^{3}+z_{\mathrm{R}}^{\omega^{2}} m^{3} \omega^{2}+z_{\mathrm{R}}^{\omega} m^{4} \omega+z_{\mathrm{R}}^{0} m^{5}}{m^{2}(m+2 \omega)}, \\
& z_{\mathrm{R}}^{\mathrm{L}}=\frac{2 l_{0}+9 m}{6}\left(l_{0}-\frac{\rho \omega}{1+\beta}\right) \text {, } \\
& z^{\omega^{4}}=\frac{1}{15}-\frac{1}{15} \frac{\rho}{1+\beta}, \quad z_{\mathrm{L}}^{0}=\frac{25 \beta^{2}-49}{60 \rho^{3}}\left(1-\frac{1}{\rho}\right)-\frac{8 \beta^{2}}{15 \rho^{2}}, \\
& z_{\mathrm{L}}^{\omega^{3}}=\frac{3-\beta}{30 \rho}-\frac{3+2 \beta}{30(1+\beta)}, \quad z_{\mathrm{L}}^{\omega^{2}}=\frac{7 \beta^{2}+8 \beta-23}{30(1+\beta) \rho}-\frac{15 \beta^{2}+6 \beta-23}{30 \rho^{2}},
\end{aligned}
$$




$$
\begin{aligned}
z_{\mathrm{L}}^{\omega} & =\frac{-20 \beta^{3}+51 \beta^{2}+38 \beta-105}{60 \rho^{3}}-\frac{55 \beta^{3}+54 \beta^{2}-82 \beta-105}{60 \rho^{2}(1+\beta)}, \\
z_{\mathrm{R}}^{\omega^{4}} & =-\frac{8}{15 \rho}+\frac{1}{15} \frac{8-\beta}{1+\beta}, \quad z_{\mathrm{R}}^{\omega^{3}}=\frac{113 \beta^{2}-2 \beta-133}{30(1+\beta) \rho}-\frac{143 \beta^{2}-34 \beta-133}{30 \rho^{2}}, \\
z_{\mathrm{R}}^{\omega^{2}} & =-\frac{339 \beta^{3}-805 \beta^{2}-353 \beta+851}{60 \rho^{3}}+\frac{-760 \beta^{3}-825 \beta^{2}+778 \beta+851}{60 \rho^{2}(1+\beta)}, \\
z_{\mathrm{R}}^{\omega} & =\frac{\beta((433-45 \beta) \beta+44)-439}{30 \rho^{3}}+\frac{\beta(\beta(9 \beta(33 \beta+3)-730)-29)+439}{30 \rho^{4}}, \\
z_{\mathrm{R}}^{0} & =\frac{270 \beta^{2}-269}{60 \rho^{3}}+\frac{309 \beta^{4}-839 \beta^{2}+538}{120 \rho^{4}},
\end{aligned}
$$

where $l_{0}=m+\omega-E^{\prime}$ and $\omega^{\prime}=l_{0}$. Our result agrees numerically with Refs. [29,38]. Integrated over the electron energy, it agrees with the total cross section of Appendix K.

\section{APPENDIX I: ELECTROMAGNETIC ENERGY SPECTRUM BELOW ELECTRON END POINT}

For the remaining nonfactorizable contribution to the electromagnetic energy spectrum $\mathrm{d} \sigma_{\mathrm{NF}}^{\nu_{\ell} e \rightarrow \nu_{\ell} e \gamma}$, it is convenient to express the result as

$$
\mathrm{d} \sigma_{\mathrm{NF}}^{\nu_{\ell} e \rightarrow \nu_{\ell} e \gamma}=\frac{\alpha}{\pi} \delta_{\gamma} \mathrm{d} \sigma_{\mathrm{LO}}^{\nu_{\ell} e \rightarrow \nu_{\ell} e}+\left(\mathrm{d} \sigma_{\mathrm{NF}}^{\nu_{\ell} e \rightarrow \nu_{\ell} e \gamma}\right)^{\prime},
$$

where in the first term the cross section of the elastic process is expressed as a function of the final state neutrino energy, and

$$
\begin{aligned}
\delta_{\gamma}= & \frac{1}{2 \beta} \ln \frac{1-\beta}{1+\beta}\left(1+\ln \frac{\rho^{17 / 2}}{4 \beta^{4}(1-\beta)^{9 / 2}}\right)-1-2 \ln \frac{1-\rho}{\rho} \\
& -\frac{1}{\beta}\left(\operatorname{Li}_{2} \frac{-\rho^{3}}{(1+\beta)^{3}}+\frac{1}{2} \operatorname{Li}_{2} \frac{1-\beta}{1+\beta}-\mathrm{Li}_{2} \frac{\rho}{1+\beta}+\frac{\pi^{2}}{6}\right) .
\end{aligned}
$$

As for the electron energy spectrum, individual corrections contain double logarithms,

$$
\begin{array}{lc}
\delta_{v} \underset{\beta \rightarrow 1}{\sim}-\frac{1}{8} \ln ^{2}(1-\beta), & \quad \underset{s}{\delta_{\beta \rightarrow 1}^{\sim}}-\frac{1}{4} \ln ^{2}(1-\beta), \\
\delta_{\text {III }}^{\sim} \frac{1}{\beta \rightarrow 1} \frac{1}{2} \ln ^{2}(1-\beta), & \delta_{\gamma \rightarrow 1}^{\sim}-\frac{1}{8} \ln ^{2}(1-\beta),
\end{array}
$$

but the complete electromagnetic energy spectrum is free from Sudakov double logarithms [118]. The residual nonfactorizable piece of the bremsstrahlung contribution, $\left(\mathrm{d} \sigma_{\mathrm{NF}}^{\nu_{e} e \rightarrow \nu_{\ell} e \gamma}\right)^{\prime}$ is given by the following substitutions in Eqs. (58) and (59):

$$
\tilde{\mathrm{I}}_{i} \rightarrow \frac{\pi^{2}}{\omega^{3}}\left(a_{i}+b_{i} \ln \frac{1+\beta}{1-\beta}+c_{i} \ln \frac{2-\rho}{1-\beta}\right) \mathrm{d} \omega^{\prime},
$$

where coefficients $a_{i}, b_{i}$, and $c_{i}$ can be expressed in terms of the initial and final neutrino energies, $\omega$ and $\omega^{\prime}$, respectively, in the following form:

$$
\begin{aligned}
& f_{\mathrm{L}}(\omega)=f^{\omega^{2}} \omega^{2}+f^{\omega} m \omega+f^{0} m^{2}, \\
& f_{\mathrm{R}}(\omega)=f_{\mathrm{L}}\left(-\omega^{\prime}\right)=f^{\omega^{2}} \omega^{\prime 2}-f^{\omega} m \omega^{\prime}+f^{0} m^{2},
\end{aligned}
$$

with dimensionless coefficients,

$$
\begin{aligned}
c^{\omega^{2}}= & \frac{3 \beta^{2}+1}{3 \rho^{3}}-\frac{7 \beta^{2}+8}{3 \rho^{2}}, \\
c^{\omega}= & \frac{2\left(\beta^{2}+4\right)}{\rho^{3}}+\frac{17 \beta^{4}+22 \beta^{2}-55}{8 \rho^{4}}, \\
c^{0}= & \frac{112-15 \beta^{4}-85 \beta^{2}}{15 \rho^{5}}+\frac{31 \beta^{4}+118 \beta^{2}-449}{60 \rho^{4}}, \\
b^{\omega^{2}}= & \frac{(\beta-3)(2 \beta-1) \rho}{6(1-\beta)^{2} \beta}+\frac{\beta+14}{6(1-\beta)}, \\
b^{\omega}= & \frac{((\beta-4) \beta-2) \rho}{2(1-\beta)^{2} \beta}+\frac{55-\beta(17 \beta+30)}{16(1-\beta)^{2}}+\frac{1}{\beta}, \\
b^{0}= & \frac{\rho(\beta(\beta+1)(23-2 \beta)-45)}{30(1-\beta)^{3} \beta} \\
& +\frac{-31 \beta^{3}-88 \beta^{2}+89 \beta+180}{120(1-\beta)^{2} \beta}, \\
a^{\omega^{2}}= & \frac{\rho\left(11 \beta^{2}+21\right)}{3\left(\beta^{4}+2 \beta^{2}-3\right)}+\frac{2\left(3 \beta^{4}+8 \beta^{2}-15\right)}{3\left(\beta^{4}+2 \beta^{2}-3\right)}, \\
a^{\omega}= & \frac{23 \beta^{4}+34 \beta^{2}-73}{4 \rho^{3}\left(\beta^{2}+3\right)}+\frac{-2 \beta^{4}+13 \beta^{2}+73}{-4 \beta^{4}-8 \beta^{2}+12}, \\
a^{0}= & \frac{85 \beta^{2}-163}{30 \rho^{3}}+\frac{15 \beta^{4}-166 \beta^{2}+163}{30 \rho^{4}} .
\end{aligned}
$$

The interference part of the energy spectrum is determined by 


$$
\begin{aligned}
& a_{\mathrm{R}}^{\mathrm{L}}=\left(-\frac{\rho}{2-\rho} \frac{2 \omega \omega^{\prime}}{m^{2}}-\frac{1}{3 \rho}+4\right) \omega^{2} \mathrm{I}_{\mathrm{R}}^{\mathrm{L}}, \\
& b_{\mathrm{R}}^{\mathrm{L}}=\left(\frac{1+\beta}{2 \beta} \frac{2 \omega \omega^{\prime}}{m^{2}}-\frac{1}{3 \beta^{2}}+\frac{1}{3(1-\beta)}+\frac{7}{6}-\frac{1+\beta}{\rho}\left(\frac{1}{3 \beta^{2}}+\frac{7}{6 \beta}+\frac{1}{6}\right)\right) \omega^{2} \mathrm{I}_{\mathrm{R}}^{\mathrm{L}}, \\
& c_{\mathrm{R}}^{\mathrm{L}}=\left(-\frac{2 \omega \omega^{\prime}}{m^{2}}+\frac{\beta^{4}-5 \beta^{2}+2}{3 \beta^{2} \rho^{2}}+\frac{2\left(1+4 \beta^{2}\right)}{3 \beta^{2} \rho}\right) \omega^{2} \mathrm{I}_{\mathrm{R}}^{\mathrm{L}},
\end{aligned}
$$

where $\mathrm{I}_{\mathrm{R}}^{\mathrm{L}}$ is given by Eq. (20).

Our result agrees with the numerical evaluation in Ref. [29].

\section{APPENDIX J: ELECTROMAGNETIC ENERGY SPECTRUM ABOVE ELECTRON END POINT}

The electromagnetic energy spectrum above the electron end point can be conveniently expressed as a sum of the factorizable and nonfactorizable corrections,

$$
\mathrm{d} \sigma^{\nu_{\ell} e \rightarrow \nu_{\ell} e \gamma}=\frac{\alpha}{\pi} \delta_{\gamma} \mathrm{d} \sigma_{\mathrm{LO}}^{\nu_{\ell} e \rightarrow \nu_{\ell} e}+\left(\mathrm{d} \sigma^{\nu_{\ell} e \rightarrow \nu_{\ell} e \gamma}\right)^{\prime}
$$

The factorizable part is given by

$$
\begin{aligned}
\delta_{\gamma}= & \frac{1}{\beta}\left(-\frac{\pi^{2}}{3}+\frac{7}{8} \ln ^{2} \frac{1+\beta}{1-\beta}+2 \ln \left(1+\frac{2 \omega}{m}\right) \ln \frac{1+\beta}{1-\beta}-\frac{3}{2} \ln \frac{1+\beta}{1-\beta} \ln \frac{2-\rho}{1-\beta}+2 \mathrm{Li}_{2} \frac{\rho}{1+\beta}\right. \\
& +\ln \frac{2-\rho\left(1+\frac{2 \omega}{m}\right)}{\rho\left(1+\frac{2 \omega}{m}\right)} \ln \left(\frac{1+\beta}{1-\beta} \frac{1+\beta-\rho\left(1+\frac{2 \omega}{m}\right)}{-1+\beta+\rho\left(1+\frac{2 \omega}{m}\right)}\right)-\mathrm{Li}_{2} \frac{\rho\left(1+\frac{2 \omega}{m}\right)}{1+\beta}-\mathrm{Li}_{2} \frac{2-\rho\left(1+\frac{2 \omega}{m}\right)}{1+\beta} \\
& \left.+\mathrm{Li}_{2} \frac{2-\rho}{1+\beta}+\Re\left(\operatorname{Li}_{2} \frac{\rho\left(1+\frac{2 \omega}{m}\right)}{1-\beta}+\mathrm{Li}_{2} \frac{2-\rho\left(1+\frac{2 \omega}{m}\right)}{1-\beta}-\mathrm{Li}_{2} \frac{2-\rho}{1-\beta}\right)\right)+2 \ln \left(\frac{2-\rho\left(1+\frac{2 \omega}{m}\right)}{1-\rho} \frac{2 \omega \omega^{\prime}+m\left(\omega^{\prime}-\omega\right)}{-m^{2}}\right),
\end{aligned}
$$

where the elastic cross section $\mathrm{d} \sigma_{\mathrm{LO}}^{\nu_{\ell} e \rightarrow \nu_{\ell} e}$ is expressed in terms of $\omega^{\prime}$. The nonfactorizable part is given by the following substitutions in Eqs. (58) and (59):

$$
\tilde{\mathrm{I}}_{i} \rightarrow \frac{\pi^{2}}{\omega^{3}}\left(a_{i}+b_{i} \ln \frac{2-\rho\left(1+\frac{2 \omega}{m}\right)}{\rho}+c_{i} \ln \left(1+\frac{2 \omega}{m}\right)+d_{i} \ln \frac{2-\rho\left(1+\frac{2 \omega}{m}\right)}{2-\rho}\right) \mathrm{d} \omega^{\prime},
$$

with coefficients $a_{i}, b_{i}, c_{i}$, and $d_{i}$,

$$
\begin{aligned}
a_{\mathrm{L}}= & \frac{\omega\left(\frac{30 m^{4}(2 \omega+m)}{m+2 \omega-2 \omega^{\prime}}-\frac{15 m^{5}}{m-2 \omega^{\prime}}-15 m^{4}+4\left(109 m^{2}+78 m \omega+2 \omega^{2}\right) \omega^{\prime 2}-2(m-2 \omega)\left(11 m^{2}+4 \omega^{2}\right) \omega^{\prime}\right)}{120 m^{3}} \\
& +\frac{\omega \omega^{\prime 3}\left(8 \omega-7 m-4 \omega^{\prime}\right)}{5 m^{3}}, \\
b_{\mathrm{L}}= & \frac{2 \omega^{5}}{15 m^{3}}-\frac{\omega^{3}}{3 m}-2 \omega^{2}, \\
c_{\mathrm{L}}= & -b_{\mathrm{L}}+\frac{m^{2}}{60}-\frac{9 m \omega}{8}-\frac{11 \omega^{2}}{3}, \\
d_{\mathrm{L}}= & \frac{m^{2}\left(6 \omega^{\prime 2}+6 \omega \omega^{\prime}-5 \omega^{2}\right)+m\left(\omega-\omega^{\prime}\right)\left(\omega^{2}+7 \omega \omega^{\prime}+13 \omega^{\prime 2}\right)+3 \omega^{\prime 2}\left(\omega-\omega^{\prime}\right)^{2}}{3 m^{2}} \\
& +\frac{2 m^{5}-135 m^{4} \omega-16\left(\omega-\omega^{\prime}\right)^{3}\left(\omega^{2}+3 \omega \omega^{\prime}+6 \omega^{\prime 2}\right)}{120 m^{3}},
\end{aligned}
$$




$$
\begin{aligned}
& a_{\mathrm{R}}=a_{\mathrm{L}}\left(\omega \leftrightarrow-\omega^{\prime}\right), \\
& b_{\mathrm{R}}=b_{\mathrm{L}}\left(\omega \leftrightarrow \omega^{\prime}\right)+b_{\mathrm{L}}\left(\omega \leftrightarrow-\omega^{\prime}\right)+c_{\mathrm{L}}\left(\omega \leftrightarrow-\omega^{\prime}\right)-d_{\mathrm{L}}\left(\omega \leftrightarrow-\omega^{\prime}\right)+2 \omega^{\prime 2}, \\
& c_{\mathrm{R}}=d_{\mathrm{R}}+\frac{\omega^{\prime 3}}{3 m}-\frac{2 \omega^{\prime 5}}{15 m^{3}}, \\
& d_{\mathrm{R}}=d_{\mathrm{L}}\left(\omega \leftrightarrow-\omega^{\prime}\right), \\
& a_{\mathrm{R}}^{\mathrm{L}}=\frac{4 \omega \omega^{\prime}\left(\left(\omega-\omega^{\prime}-3 m\right)^{2}-13 m^{2}\right)}{3 m\left(2 E_{\mathrm{EM}}-m\right)}, \\
& b_{\mathrm{R}}^{\mathrm{L}}=2\left(\frac{\omega^{3}}{3 m}-m \omega^{\prime}-\omega^{2}+\omega \omega^{\prime}\right), \\
& c_{\mathrm{R}}^{\mathrm{L}}=\frac{2 m^{2}}{3}-\frac{2 \omega^{3}}{3 m}+m\left(3 \omega-\omega^{\prime}\right)+2 \omega\left(\omega-\omega^{\prime}\right), \\
& d_{\mathrm{R}}^{\mathrm{L}}=c_{\mathrm{R}}^{\mathrm{L}}+\frac{2}{3} \omega^{\prime}\left(\frac{\omega^{\prime 2}}{m}-3 E_{\mathrm{EM}}\right),
\end{aligned}
$$

where $E_{\mathrm{EM}}=m+\omega-\omega^{\prime}$, and as explained in Sec. II B $\mathrm{d} \sigma / \mathrm{d} E^{\prime}=\mathrm{d} \sigma / \mathrm{d} \omega^{\prime}$. Our result agrees with a numerical evaluation of Ref. [29]. The total cross section from both regions of Secs. IV I 1 and IV I 2 is in agreement with Ref. [31]. Correcting obvious typos, the function $\tilde{\mathrm{I}}_{\mathrm{L}}^{\mathrm{R}}$ and only the function $\tilde{\mathrm{I}}_{\mathrm{L}}$ of Eq. (J3) with the interchange $\tilde{\mathrm{I}}_{\mathrm{L}} \leftrightarrow \tilde{\mathrm{I}}_{\mathrm{R}}$ are in agreement with Ref. [31]. For all other kinematical factors of Secs. IV I 1 and IV I 2, we find nontrivial discrepancies with Ref. [31].

\section{APPENDIX K: ABSOLUTE CROSS SECTION}

The total cross-section correction including both real and virtual contributions, besides the closed fermion loop correction of Secs. III B and III C, is given by the following substitutions in Eqs. (58) and (59) [31]:

$$
\begin{aligned}
\frac{\tilde{\mathrm{I}}_{\mathrm{L}}}{\pi^{2}} \rightarrow & (1+R) \mathrm{L}_{2}+\frac{r^{2}(1-r)}{2} \ln ^{2} R+4(1-R) \ln r-\left(r^{2}-\frac{r}{2}+\frac{3 R}{2}+\frac{10}{3}\right) \ln R-\frac{r}{2}+\frac{19(1-R)}{24}, \\
\frac{\tilde{\mathrm{I}}_{\mathrm{R}}}{\pi^{2}} \rightarrow & -4 r^{2}(2 r+1) \mathrm{L}_{3}+\left(8 r^{2}+\frac{R^{3}}{3}+2 R+\frac{1}{3}\right) \mathrm{L}_{2}-\frac{7 r^{3}}{6} \ln ^{2} R+\left(8 r-\frac{8 R^{3}}{9}+\frac{R^{2}}{3}-\frac{16 R}{3}+\frac{17}{9}\right) \ln r \\
& -\left(\frac{31 r^{2}}{3}-\frac{7 r}{3}-\frac{R^{3}}{18}+\frac{35 R}{6}+\frac{5}{3}\right) \ln R-\frac{7 r}{6}-\frac{11 R^{3}}{8}+\frac{13 R^{2}}{12}+\frac{73 R}{36}+\frac{43}{72}, \\
\frac{\tilde{\mathrm{I}}_{\mathrm{R}}^{\mathrm{L}}}{\pi^{2}} \rightarrow & -4 r^{3} \mathrm{~L}_{3}-\left(-4 r^{2}+2 r+R^{2}-R\right) \mathrm{L}_{2}-r^{2}(2+5 r) \ln ^{2} R+\left(4 r+3 R^{2}-7 R\right) \ln r \\
& +7\left(-2 r^{2}+r-R\right) \ln R-5 r+\frac{13}{4} R^{2}+\frac{15}{4} R,
\end{aligned}
$$

with additional definitions,

$$
\begin{aligned}
\mathrm{L}_{2}= & \frac{\mathrm{Li}_{2}\left(1-1 / R^{2}\right)-\mathrm{Li}_{2}\left(1-R^{2}\right)}{2}+\Re\left(\mathrm{Li}_{2}\left(1+\frac{1}{R}\right)-\mathrm{Li}_{2}(1+R)\right)+\mathrm{Li}_{2}\left(-\frac{1}{r}\right)+2 \ln R \ln r, \\
\mathrm{~L}_{3}= & \frac{\mathrm{Li}_{3}\left(1-1 / R^{2}\right)+\mathrm{Li}_{3}\left(1-R^{2}\right)}{2}+2\left(\mathrm{Li}_{2}(-R)+\frac{1}{2} \mathrm{Li}_{2}\left(R^{2}\right)\right) \ln R-\mathrm{Li}_{2}\left(-\frac{1}{r}\right) \ln R-\ln { }^{2} R \ln r \\
& -\frac{\mathrm{Li}_{3}\left(R^{2}\right)}{4}-\mathrm{Li}_{3}(-R)-\mathrm{Li}_{3}\left(-\frac{1}{R}\right)+\ln \left[\left(1-R^{2}\right)(1+R)\right] \ln ^{2} R-\pi^{2} \ln \frac{1+R}{2 \sqrt{R}}-\zeta(3) \\
& +\Re\left(2\left(\mathrm{Li}_{3}\left(1+\frac{1}{R}\right)+\mathrm{Li}_{3}(1+R)\right)-\frac{1}{4} \mathrm{Li}_{3}\left(\frac{1}{R^{2}}\right)-4 \mathrm{Li}_{3}(2)\right), \\
R= & \frac{m}{m+2 \omega}, \quad \quad \quad r=\frac{m}{2 \omega} .
\end{aligned}
$$


Note also that the total elastic cross section at leading order is given by the following substitutions in Eqs. (14) and (15),

$$
\int \mathrm{d} \omega^{\prime} \mathrm{I}_{\mathrm{L}} \rightarrow \omega(1-R), \quad \int \mathrm{d} \omega^{\prime} \mathrm{I}_{\mathrm{R}} \rightarrow \frac{\omega\left(1-R^{3}\right)}{3}, \quad \int \mathrm{d} \omega^{\prime} \mathrm{I}_{\mathrm{R}}^{\mathrm{L}} \rightarrow-\frac{\omega R^{2}}{r} .
$$

The "dynamical" correction of Secs. III B and III C to the total unpolarized cross section, $\sigma_{\mathrm{dyn}}^{\nu_{e} e \rightarrow \nu_{\ell} e}$, can be expressed in the following form:

$$
\sigma_{\mathrm{dyn}}^{\nu_{\ell} e \rightarrow \nu_{\ell} e}=\frac{\alpha}{\pi} \sum_{\ell^{\prime}} \tilde{\sigma}_{\mathrm{dyn}, \ell^{\prime}}^{\nu_{\ell} e \rightarrow \nu_{\ell} e}+\frac{\alpha}{\pi}\left(\hat{\Pi}_{3 \gamma}^{(3)}(0)-2 \sin ^{2} \theta_{W} \hat{\Pi}_{\gamma \gamma}^{(3)}(0)+\frac{Q_{u}\left(c_{\mathrm{L}}^{u}+c_{\mathrm{R}}^{u}\right)}{2 \sqrt{2} \mathrm{G}_{\mathrm{F}}} N_{c} \Pi\left(0, m_{c}\right)\right) \tilde{\sigma}_{\mathrm{dyn}, q}^{\nu_{\ell} e \rightarrow \nu_{\ell} e} .
$$

The reduced cross section due to the lepton $\ell^{\prime}$ loop contribution $\tilde{\sigma}_{\mathrm{dyn}, \ell^{\prime}}^{\nu_{\ell} e \rightarrow \nu_{\ell} e}$ is obtained by replacements of Eqs. (43)-(45) and the following substitutions in Eqs. (14) and (15),

$$
\begin{aligned}
Q_{\ell^{\prime}} \int \mathrm{d} \omega^{\prime} \Pi\left(q^{2}, m_{\ell^{\prime}}\right) \mathrm{I}_{\mathrm{L}} \rightarrow & -\frac{\omega(1-R)}{3} \ln \frac{\mu^{2}}{m_{\ell^{\prime}}^{2}}+\frac{\omega R}{3 r}\left(R_{l}^{3} \ln \frac{R_{l}+1}{R_{l}-1}-2 R_{l}^{2}-\frac{2}{3}\right), \\
Q_{\ell^{\prime}} \int \mathrm{d} \omega^{\prime} \Pi\left(q^{2}, m_{\ell^{\prime}}\right) \mathrm{I}_{\mathrm{R}} \rightarrow & -\frac{\omega\left(1-R^{3}\right)}{9} \ln \frac{\mu^{2}}{m_{\ell^{\prime}}^{2}}+\frac{m R^{3} R_{l}^{3}}{24 r^{4}}\left(\frac{3 m^{2}+15 m \omega+25 \omega^{2}}{3 \omega^{2}}-R_{l}^{2}\right) \ln \frac{R_{l}+1}{R_{l}-1} \\
& +\frac{\omega R^{3}}{8 r^{2}}\left(1+\frac{5+R_{l}^{2}}{6 r}\right)\left(\left(R_{l}^{2}-1\right)^{2} \ln ^{2} \frac{R_{l}+1}{R_{l}-1}-4 R_{l} \ln \frac{R_{l}+1}{R_{l}-1}\right)+\frac{m R^{3} R_{l}^{4}}{24 r^{4}} \\
& -\frac{\omega R^{3}}{18 r^{3}}\left(\frac{6 m^{2}+39 m \omega+53 \omega^{2}}{2 \omega^{2}} R_{l}^{2}+\frac{m^{2}-10 m \omega-18 \omega^{2}}{\omega^{2}}\right), \\
Q_{\ell^{\prime}} \int \mathrm{d} \omega^{\prime} \Pi\left(q^{2}, m_{\ell^{\prime}}\right) \mathrm{I}_{\mathrm{R}}^{\mathrm{L}} \rightarrow & \frac{\omega R^{2}}{3 r} \ln \frac{\mu^{2}}{m_{\ell^{\prime}}^{2}}+\frac{\omega R^{2}}{24 r}\left(3\left(R_{l}^{2}-1\right)^{2} \ln ^{2} \frac{R_{l}+1}{R_{l}-1}+4\left(R_{l}^{2}-3\right) R_{l} \ln \frac{R_{l}+1}{R_{l}-1}\right) \\
& +\frac{\omega R^{2}}{24 r}\left(\frac{112}{3}-20 R_{l}^{2}\right),
\end{aligned}
$$

with the vanishing in the limit $R_{l} \rightarrow \infty$ terms, beyond the first $\mu$-dependent contributions, where

$$
R_{l}=\sqrt{1+4 \frac{m_{\ell^{\prime}}^{2}}{m^{2}} \frac{r^{2}}{R}} .
$$

The reduced cross section arising from the quark loop contributions $\tilde{\sigma}_{\text {dyn }, q}^{\nu_{\ell} e \rightarrow \nu_{\ell} e}$ is obtained replacing $\nu_{\ell} e$ couplings in Eqs. (14) and (15) as

$$
\begin{aligned}
\int \mathrm{d} \omega^{\prime}\left(c_{\mathrm{L}}^{\nu_{\ell} \ell^{\prime}}\right)^{2} \mathrm{I}_{\mathrm{L}} & \rightarrow 2 \sqrt{2} \mathrm{G}_{\mathrm{F}} c_{\mathrm{L}}^{\nu_{\ell} \ell^{\prime}} \omega(1-R), \\
\int \mathrm{d} \omega^{\prime} c_{\mathrm{R}}^{2} \mathrm{I}_{\mathrm{R}} & \rightarrow 2 \sqrt{2} \mathrm{G}_{\mathrm{F}} c_{\mathrm{R}} \frac{\omega\left(1-R^{3}\right)}{3}, \\
\int \mathrm{d} \omega^{\prime} c_{\mathrm{L}}^{\nu} \ell^{\prime} c_{\mathrm{R}} \mathrm{I}_{\mathrm{R}}^{\mathrm{L}} & \rightarrow-\sqrt{2} \mathrm{G}_{\mathrm{F}}\left(c_{\mathrm{L}}^{\nu} \ell^{\prime}+c_{\mathrm{R}}\right) \frac{\omega R^{2}}{r} .
\end{aligned}
$$

\section{APPENDIX L: AVERAGED OVER FLUX NEUTRINO CROSS SECTIONS}

In the following, we average the energy spectrum with anticipated flux profiles of the DUNE Near Detector

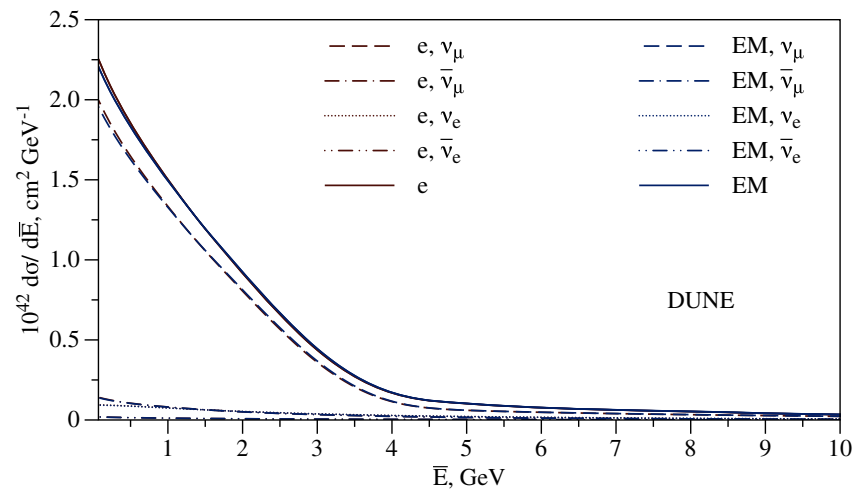

FIG. 13. Electron (e) and electromagnetic (EM) energy spectra in elastic neutrino-electron scattering for the neutrino beam mode of DUNE experiments. The electron energy spectrum is above at low energy. Electron and muon (anti)neutrino contributions are shown. 


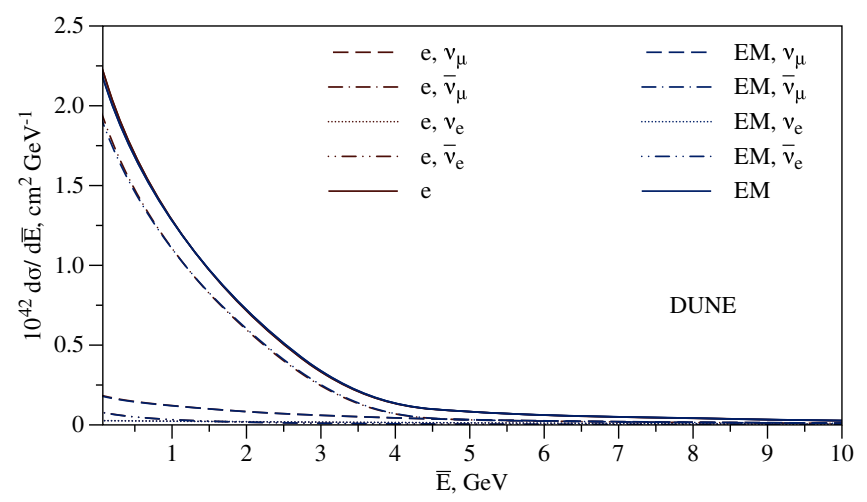

FIG. 14. Same as Fig. 13 for the antineutrino beam mode.

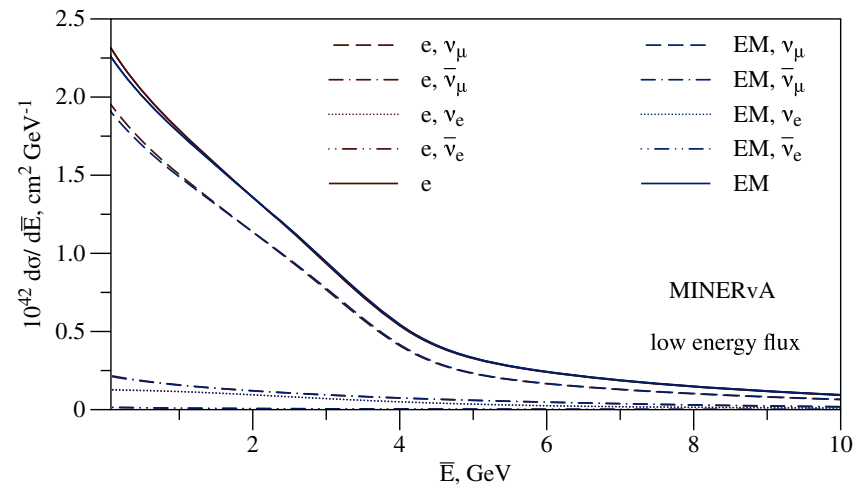

FIG. 15. Same as Fig. 13 for the MINERvA experiment.

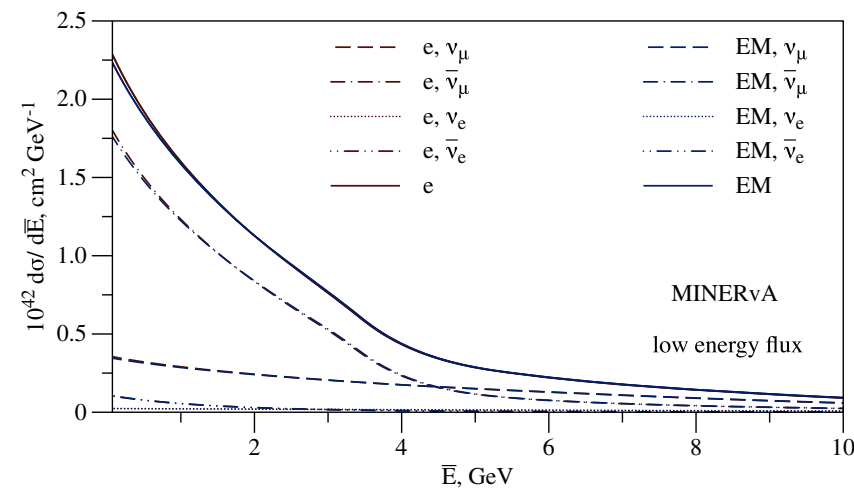

FIG. 16. Same as Fig. 14 for the MINERvA experiment.

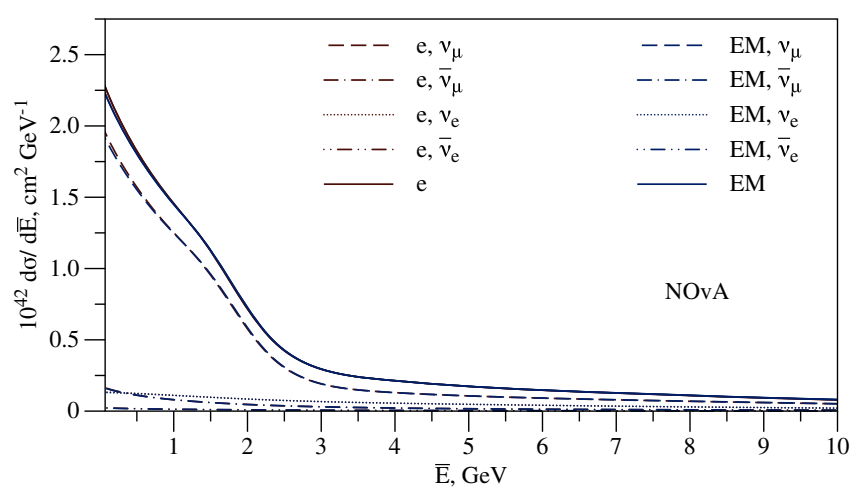

FIG. 17. Same as Fig. 13 for the NOvA experiment.

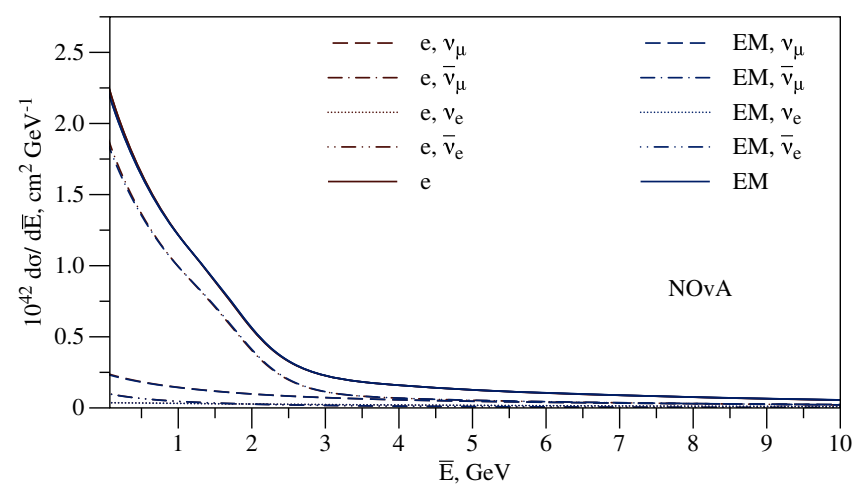

FIG. 18. Same as Fig. 14 for the NOvA experiment.

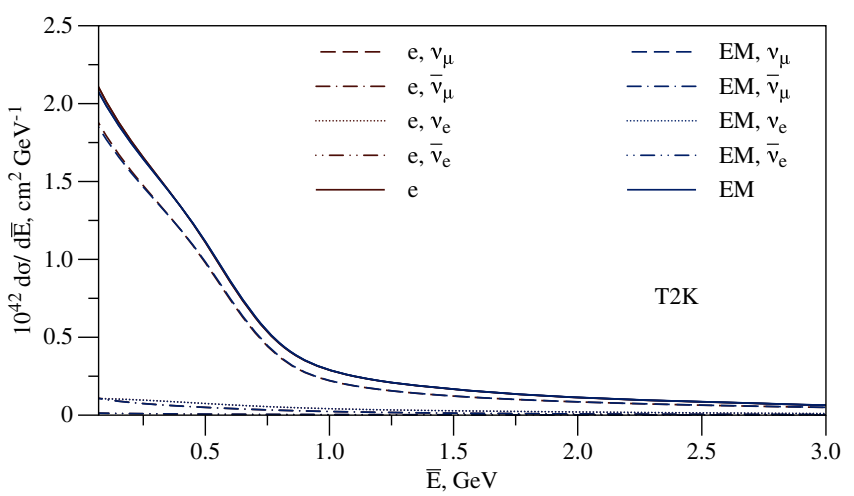

FIG. 19. Same as Fig. 13 for the T2K experiment.

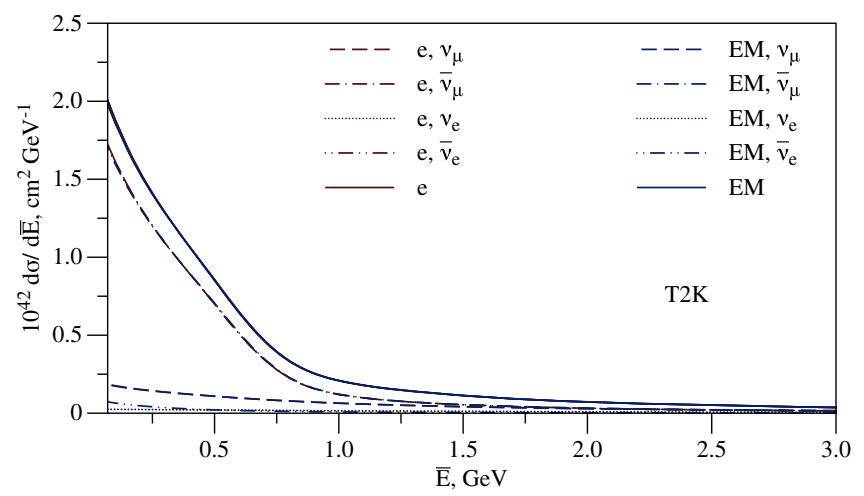

FIG. 20. Same as Fig. 14 for the T2K experiment.

$[141,142]$ at Fermilab. In Figs. 13 and 14, we show the resulting electron and electromagnetic energy spectra for neutrino and antineutrino beam modes.

The corresponding figures for MINERvA [19,20,143145], NOvA [146], and T2K [147,148] experiments are shown in Figs. 15-20. The difference between the electron and electromagnetic energy spectra slightly washes out after averaging over the typical neutrino flux. It is larger at low energies, where it can reach an effect of the relative order $1 \%-3 \%$, and smaller at higher energies reflecting the dependence in Fig. 11. 
[1] B. T. Cleveland, T. Daily, R. Davis, Jr., J. R. Distel, K. Lande, C. K. Lee, P. S. Wildenhain, and J. Ullman, Astrophys. J. 496, 505 (1998).

[2] W. Hampel et al. (GALLEX Collaboration), Phys. Lett. B 447, 127 (1999).

[3] Q. R. Ahmad et al. (SNO Collaboration), Phys. Rev. Lett. 89, 011301 (2002).

[4] J. N. Abdurashitov et al. (SAGE Collaboration), Zh. Eksp. Teor. Fiz. 122, 211 (2002) [J. Exp. Theor. Phys. 95, 181 (2002)].

[5] S. Fukuda et al. (Super-Kamiokande Collaboration), Phys. Rev. Lett. 86, 5651 (2001).

[6] S. N. Ahmed et al. (SNO Collaboration), Phys. Rev. Lett. 92, 181301 (2004).

[7] Y. Fukuda et al. (Super-Kamiokande Collaboration), Phys. Rev. Lett. 81, 1562 (1998).

[8] Y. Ashie et al. (Super-Kamiokande Collaboration), Phys. Rev. Lett. 93, 101801 (2004).

[9] K. Eguchi et al. (KamLAND Collaboration), Phys. Rev. Lett. 90, 021802 (2003).

[10] T. Araki et al. (KamLAND Collaboration), Phys. Rev. Lett. 94, 081801 (2005).

[11] Y. Abe et al. (Double Chooz Collaboration), Phys. Rev. D 86, 052008 (2012).

[12] J. K. Ahn et al. (RENO Collaboration), Phys. Rev. Lett. 108, 191802 (2012).

[13] F. P. An et al. (Daya Bay Collaboration), Chin. Phys. C 37, 011001 (2013).

[14] M. H. Ahn et al. (K2K Collaboration), Phys. Rev. Lett. 90, 041801 (2003).

[15] D. G. Michael et al. (MINOS Collaboration), Phys. Rev. Lett. 97, 191801 (2006).

[16] K. Abe et al. (T2K Collaboration), Phys. Rev. D 88, 032002 (2013).

[17] B. Pontecorvo, Zh. Eksp. Teor. Fiz. 33, 549 (1957) [Sov. Phys. JETP 6, 429 (1957)].

[18] B. Pontecorvo, Zh. Eksp. Teor. Fiz. 53, 1717 (1967) [Sov. Phys. JETP 26, 984 (1968)].

[19] J. Park et al. (MINERvA Collaboration), Phys. Rev. D 93, 112007 (2016).

[20] E. Valencia et al. (MINERvA Collaboration), Phys. Rev. D 100, 092001 (2019).

[21] T. D. Lee and A. Sirlin, Rev. Mod. Phys. 36, 666 (1964).

[22] M. Ram, Phys. Rev. 155, 1539 (1967).

[23] S. Weinberg, Phys. Rev. Lett. 19, 1264 (1967).

[24] G. 't Hooft, Phys. Lett. 37B, 195 (1971).

[25] S. Sarantakos, A. Sirlin, and W. J. Marciano, Nucl. Phys. B217, 84 (1983).

[26] J. N. Bahcall, M. Kamionkowski, and A. Sirlin, Phys. Rev. D 51, 6146 (1995).

[27] D. Yu. Bardin and V. A. Dokuchaeva, Nucl. Phys. B246, 221 (1984).

[28] D. Yu. Bardin and V. A. Dokuchaeva, Yad. Fiz. 43, 1513 (1986) [Sov. J. Nucl. Phys. 43, 975 (1986)].

[29] M. Passera, Phys. Rev. D 64, 113002 (2001).

[30] M. Green, J. Phys. G 7, 1169 (1981).

[31] D. Yu. Bardin and V. A. Dokuchaeva, Yad. Fiz. 39, 888 (1984) [Sov. J. Nucl. Phys. 39, 563 (1984)].

[32] P. Salomonson and Y. Ueda, Phys. Rev. D 11, 2606 (1975).
[33] E. D. Zhizhin, R. V. Konoplich, and Yu. P. Nikitin, Izv. Vuz. Fiz. 1975, 82 (1975).

[34] N. Byers, R. Ruckl, and A. Yano, Physica (Amsterdam) 96A, 163 (1979).

[35] M. Green and M. J. G. Veltman, Nucl. Phys. B169, 137 (1980); B175, 547(E) (1980).

[36] W. J. Marciano and A. Sirlin, Phys. Rev. D 22, 2695 (1980); 31, 213(E) (1985).

[37] K.-i. Aoki, Z. Hioki, R. Kawabe, M. Konuma, and T. Muta, Prog. Theor. Phys. 65, 1001 (1981).

[38] K.-i. Aoki and Z. Hioki, Prog. Theor. Phys. 66, 2234 (1981).

[39] Z. Hioki, Prog. Theor. Phys. 67, 1165 (1982).

[40] A. M. Mourao, L. Bento, and B. K. Kerimov, Phys. Lett. B 237, 469 (1990).

[41] A. Weber and L. M. Sehgal, Nucl. Phys. B359, 262 (1991).

[42] F. Buccella, C. Gualdi, G. Miele, and P. Santorelli, Nuovo Cimento B 107, 1343 (1992).

[43] J. Bernabeu, S. M. Bilenky, F. J. Botella, and J. Segura, Nucl. Phys. B426, 434 (1994).

[44] E. Akhmedov, G. Arcadi, M. Lindner, and S. Vogl, J. High Energy Phys. 10 (2018) 045.

[45] W. J. Marciano and Z. Parsa, J. Phys. G 29, 2629 (2003).

[46] A. Sirlin and A. Ferroglia, Rev. Mod. Phys. 85, 263 (2013).

[47] R. J. Hill and O. Tomalak, arXiv:1911.01493.

[48] D. Akimov et al. (COHERENT Collaboration), Science 357, 1123 (2017).

[49] D. S. Ayres et al. (NOvA Collaboration), Fermi National Accelerator Lab. (FNAL), Technical Report No. FERMILAB-DESIGN-2007-01, TRN: US0804659, 2007.

[50] R. Acciarri et al. (DUNE Collaboration), arXiv:1512 .06148 .

[51] K. Abe et al., arXiv:1109.3262.

[52] K. Abe et al. (Super-Kamiokande Collaboration), Phys. Rev. D 83, 052010 (2011).

[53] M. Wurm et al. (LENA Collaboration), Astropart. Phys. 35, 685 (2012).

[54] Y.-F. Li, J. Cao, Y. Wang, and L. Zhan, Phys. Rev. D 88, 013008 (2013).

[55] C. Giunti and A. Studenikin, Rev. Mod. Phys. 87, 531 (2015).

[56] C. Giunti, K. A. Kouzakov, Y.-F. Li, A. V. Lokhov, A. I. Studenikin, and S. Zhou, Ann. Phys. (Berlin) 528, 198 (2016).

[57] E. Fermi, Z. Phys. 88, 161 (1934).

[58] R. P. Feynman and M. Gell-Mann, Phys. Rev. 109, 193 (1958).

[59] E. C. G. Sudarshan and R. E. Marshak, Phys. Rev. 109, 1860 (1958).

[60] M. E. Nahmias, Math. Proc. Cambridge Philos. Soc. 31, 99 (1935).

[61] H. R. Crane, Rev. Mod. Phys. 20, 278 (1948).

[62] J. H. Barrett, Phys. Rev. 79, 907 (1950).

[63] J. L. Kulp and L. E. Tyron, Rev. Sci. Instrum. 23, 296 (1952).

[64] F. G. Houtermans and W. Thirring, Helv. Phys. Acta 27, 81 (1954).

[65] C. L. Cowan, F. Reines, and F. B. Harrison, Phys. Rev. 96, 1294 (1954). 
[66] J. Bernstein, M. Ruderman, and G. Feinberg, Phys. Rev. 132, 1227 (1963).

[67] F. J. Hasert et al., Phys. Lett. 46B, 121 (1973).

[68] F. Reines, H. S. Gurr, and H. W. Sobel, Phys. Rev. Lett. 37, 315 (1976).

[69] L. Wolfenstein, Phys. Rev. D 17, 2369 (1978).

[70] L. Wolfenstein, Phys. Rev. D 20, 2634 (1979).

[71] L. B. Okun, Leptons and Quarks (North-Holland, Amsterdam, Netherlands, 1982).

[72] R. C. Allen et al., Phys. Rev. Lett. 55, 2401 (1985).

[73] S. P. Mikheyev and A. Yu. Smirnov, Sov. J. Nucl. Phys. 42, 913 (1985).

[74] H. A. Bethe, Phys. Rev. Lett. 56, 1305 (1986).

[75] S. J. Parke, Phys. Rev. Lett. 57, 1275 (1986).

[76] S. P. Rosen and J. M. Gelb, Phys. Rev. D 34, 969 (1986).

[77] J. Dorenbosch et al. (CHARM Collaboration), Z. Phys. C 41, 567 (1989); 51, 142(E) (1991).

[78] R. C. Allen et al., Phys. Rev. Lett. 64, 1330 (1990).

[79] L. A. Ahrens et al., Phys. Rev. D 41, 3297 (1990).

[80] G. S. Vidyakin, V.N. Vyrodov, I. I. Gurevich, Yu. V. Kozlov, V. P. Martemyanov, S. V. Sukhotin, V. G. Tarasenkov, E. V. Turbin, and S. K. Khakhimov, Pis'ma Zh. Eksp. Teor. Fiz. 55, 212 (1992) [JETP Lett. 55, 206 (1992)].

[81] R. C. Allen et al., Phys. Rev. D 47, 11 (1993).

[82] A. I. Derbin, A. V. Chernyi, L. A. Popeko, V. N. Muratova, G. A. Shishkina, and S. I. Bakhlanov, Pis'ma Zh. Eksp. Teor. Fiz. 57, 755 (1993) [JETP Lett. 57, 768 (1993)].

[83] J. Horejsi, Introduction to Electroweak Unification: Standard Model from Tree Unitarity (World Scientific Publishing, Singapore, 1993).

[84] P. Vilain et al. (CHARM-II Collaboration), Phys. Lett. B 335, 246 (1994).

[85] L. B. Auerbach et al. (LSND Collaboration), Phys. Rev. D 63, 112001 (2001).

[86] D. W. Liu et al. (Super-Kamiokande Collaboration), Phys. Rev. Lett. 93, 021802 (2004).

[87] Z. Daraktchieva et al. (MUNU Collaboration), Phys. Lett. B 615, 153 (2005).

[88] A. G. Beda, V. B. Brudanin, E. V. Demidova, V. G. Egorov, M. G. Gavrilov, M. V. Shirchenko, A. S. Starostin, and T. Vylov, Phys. At. Nucl. 70, 1873 (2007).

[89] C. Arpesella et al. (Borexino Collaboration), Phys. Rev. Lett. 101, 091302 (2008).

[90] M. Deniz et al. (TEXONO Collaboration), Phys. Rev. D 81, 072001 (2010).

[91] A. G. Beda, V. B. Brudanin, V. G. Egorov, D. V. Medvedev, V. S. Pogosov, M. V. Shirchenko, and A. S. Starostin, Adv. High Energy Phys. 2012, 1 (2012).

[92] J. A. Formaggio and G. P. Zeller, Rev. Mod. Phys. 84, 1307 (2012).

[93] J. Segura, J. Bernabeu, F. J. Botella, and J. Penarrocha, Phys. Rev. D 49, 1633 (1994).

[94] J. Segura, J. Bernabeu, F. J. Botella, and J. A. Penarrocha, Phys. Lett. B 335, 93 (1994).

[95] J. Bernabeu and S. Palomares-Ruiz, J. High Energy Phys. 02 (2004) 068.

[96] J. Bernabeu, J. Papavassiliou, and M. Passera, Phys. Lett. B 613, 162 (2005).

[97] J. S. Schwinger, Phys. Rev. 76, 790 (1949).
[98] R. Barbieri, J. A. Mignaco, and E. Remiddi, Nuovo Cimento A 11, 824 (1972).

[99] W. Pauli and M. E. Rose, Phys. Rev. 49, 462 (1936).

[100] R. P. Feynman, Phys. Rev. 76, 769 (1949).

[101] Y. S. Tsai, Phys. Rev. 120, 269 (1960).

[102] M. Vanderhaeghen, J. M. Friedrich, D. Lhuillier, D. Marchand, L. Van Hoorebeke, and J. Van de Wiele, Phys. Rev. C 62, 025501 (2000).

[103] M. Heller, O. Tomalak, M. Vanderhaeghen, and S. Wu, Phys. Rev. D 100, 076013 (2019).

[104] A. Djouadi and C. Verzegnassi, Phys. Lett. B 195, 265 (1987).

[105] A. Djouadi, Nuovo Cimento A 100, 357 (1988).

[106] B. A. Kniehl, Nucl. Phys. B347, 86 (1990).

[107] S. Fanchiotti, B. A. Kniehl, and A. Sirlin, Phys. Rev. D 48, 307 (1993).

[108] F. Jegerlehner, Z. Phys. C 32, 195 (1986).

[109] F. Jegerlehner, Nuovo Cimento C 034S1, 31 (2011).

[110] J. Erler, Phys. Rev. D 59, 054008 (1999).

[111] J. Erler and M. J. Ramsey-Musolf, Phys. Rev. D 72, 073003 (2005).

[112] J. Erler and R. Ferro-Hernòndez, J. High Energy Phys. 03 (2018) 196.

[113] S. Abreu et al., in Theory report on the 11th FCC-ee workshop, edited by A. Blondel, J. Gluza, S. Jadach, P. Janot, and T. Riemann (2019) [arXiv:1905.05078].

[114] F. Bloch and A. Nordsieck, Phys. Rev. 52, 54 (1937).

[115] N. Nakanishi, Prog. Theor. Phys. 19, 159 (1958).

[116] T. Kinoshita, J. Math. Phys. (N.Y.) 3, 650 (1962).

[117] T. D. Lee and M. Nauenberg, Phys. Rev. 133, B1549 (1964).

[118] V. V. Sudakov, Zh. Eksp. Teor. Fiz. 30, 87 (1956) [Sov. Phys. JETP 3, 65 (1956)].

[119] D. R. Yennie, S. C. Frautschi, and H. Suura, Ann. Phys. (N.Y.) 13, 379 (1961).

[120] M. Tanabashi et al. (Particle Data Group), Phys. Rev. D 98, 030001 (2018).

[121] R. J. Hill, Phys. Rev. D 95, 013001 (2017).

[122] J. Bernstein and T. D. Lee, Phys. Rev. Lett. 11, 512 (1963).

[123] W. A. Bardeen, R. Gastmans, and B. E. Lautrup, Nucl. Phys. B46, 319 (1972).

[124] S. Y. Lee, Phys. Rev. D 6, 1701 (1972).

[125] A. D. Dolgov and Ya. B. Zeldovich, Rev. Mod. Phys. 53, 1 (1981).

[126] J. Bernabeu, J. Papavassiliou, and D. Binosi, Nucl. Phys. B716, 352 (2005).

[127] W. Wetzel, Z. Phys. C 11, 117 (1981).

[128] N. A. Papadopoulos, J. A. Penarrocha, F. Scheck, and K. Schilcher, Nucl. Phys. B258, 1 (1985).

[129] N. A. Papadopoulos, J. A. Penarrocha, F. Scheck, and K. Schilcher, Phys. Lett. 149B, 213 (1984).

[130] F. Burger, K. Jansen, M. Petschlies, and G. Pientka, J. High Energy Phys. 11 (2015) 215.

[131] M. Cè, A. Gèrardin, K. Ottnad, and H. B. Meyer, Proc. Sci. LATTICE2018 (2018) 137.

[132] R. J. Hill, K. S. McFarland, and O. Tomalak (to be published).

[133] R. Mertig, M. Bohm, and A. Denner, Comput. Phys. Commun. 64, 345 (1991). 
[134] V. Shtabovenko, R. Mertig, and F. Orellana, Comput. Phys. Commun. 207, 432 (2016).

[135] T. Hahn and M. Perez-Victoria, Comput. Phys. Commun. 118, 153 (1999).

[136] D. Binosi and L. Theussl, Comput. Phys. Commun. 161, 76 (2004).

[137] W. R. Inc., Mathematica, Version 11.0.1.0 (Wolfram Research, Inc., Champaign, IL, 2016).

[138] K. G. Chetyrkin, J. H. Kuhn, and A. Kwiatkowski, Phys. Rep. 277, 189 (1996).

[139] K. G. Chetyrkin, J. H. Kuhn, and M. Steinhauser, Nucl. Phys. B482, 213 (1996).

[140] K. G. Chetyrkin, B. A. Kniehl, and M. Steinhauser, Nucl. Phys. B510, 61 (1998).
[141] T. Alion et al. (DUNE Collaboration), arXiv:1606.09550.

[142] Dune fluxes, http://home.fnal.gov/ ljf26/DUNEFluxes/.

[143] J. Devan et al. (MINERvA Collaboration), Phys. Rev. D 94, 112007 (2016).

[144] L. Aliaga et al. (MINERvA Collaboration), Phys. Rev. D 94, 092005 (2016); 95, 039903(A) (2017).

[145] L. A. Soplin, Neutrino flux prediction for the NuMI beamline, Ph.D. thesis, The College of William and Mary, 2016.

[146] Nova fluxes, http://nova-docdb.fnal.gov/cgi-bin/Show Document?docid=25266.

[147] K. Abe et al. (T2K Collaboration), Phys. Rev. D 87, 012001 (2013); 87, 019902(A) (2013).

[148] K. Abe et al. (T2K Collaboration), Phys. Rev. D 91, 072010 (2015). 